\title{
CIR-Myo News: Abstracts of the 2015 Spring Padua Muscle Days
}

\author{
Terme Euganee Padua (Italy), March 12 - 14, 2015
}

\section{Abstracts}

\author{
Stimulation of denervated muscle: \\ questions answered, questions raised
}

\section{Stanley Salmons}

Department of Human Anatomy and Cell Biology, University of Liverpool, UK.

E-mail: "Salmons, Stanley" <S.Salmons@liverpool.ac.uk>

Injury to peripheral nerves is not uncommon, and spinal injury with fracture dislocation of the vertebrae can damage roots as well as the cord. Both types of trauma leave the corresponding muscles denervated, resulting in a flaccid paralysis and catastrophic loss of muscle mass. When this occurs in the lower limbs, a loss of cushioning over bony prominences, combined with a deterioration in skin condition, greatly increases the risk of developing pressure sores. Furthermore, bones become osteoporotic and the wasted appearance of the affected limbs can be a source of great distress to patients. Although there has been a longstanding interest in the potential therapeutic value of electrical stimulation of denervated muscles in humans it was always regarded as impractical. In the absence of the nerve or intramuscular nerve branches the muscles must be excited directly. The charge delivery needed for this is so high that the approach has been frustrated by regulatory restrictions and a lack of suitable equipment. More recently, however, these problems were addressed, and the value of stimulation was clearly demonstrated, in a remarkable research programme pursued with the support of the EU Commission Shared Cost Project "RISE". It is hard to explore the benefits and limitations of the technique in patient groups, which are small and inhomogeneous in age, nature and duration of injury, and compliance. Moreover, the intense surface stimulation elicits co-contraction of antagonistic muscle groups, which interferes with the measurement of force or torque. In Project "RISE", the important clinical work ${ }^{1}$ was therefore complemented by laboratory studies. The usual model, total sciatic section in the rat, is unsatisfactory on two counts. First, denervated rat muscles show evidence of extensive degeneration in a few months, ${ }^{2}$ differing in this respect from the muscles of other species. Denervated human muscles, in particular, do not undergo significant necrosis for at least a year post-injury (U. Carraro, personal communication), and we could confirm that this was also true of the rabbit. ${ }^{3}$ Second, most published studies of stimulation in the denervated rat start at, or soon after, the moment of lesion, which does not correspond to the clinical situation. The "RISE" experimental studies to be discussed were conducted by the Muscle Research Group, University of Liverpool, UK, in a long-term model of established selective denervation in the rabbit. The Department for Biomedical Engineering and Physics, University of Vienna, designed the implantable stimulator, ${ }^{4}$ ultrastructural studies were performed at the Interuniversity Institute of Myology, Chieti, and valuable input was provided by clinical colleagues at the Ludwig Boltzmann Institute of Electrostimulation and Physical Rehabilitation, Wilhelminenspital, Vienna, Austria. Through this joint programme we were able to assemble comprehensive physiological, histological, biochemical, and ultrastructural data on muscles subjected to selective denervation alone for up to 1 year, and muscles subjected to denervation and stimulation for up to 3 months. ${ }^{3,5,6}$ Although this data settled several issues, and may serve to take some of the guesswork out of the design of stimulation protocols for clinical use, it raised some tantalizing questions. These will be worth addressing in future studies.

1. Kern H, Salmons S, Mayr W, Rossini K, Carraro U. Recovery of long-term denervated human muscles induced by electrical stimulation. Muscle Nerve 2005;31:98-101.

2. Schmalbruch H, al-Amood WS, Lewis DM. Morphology of long-term denervated rat soleus muscle and the effect of chronic electrical stimulation. J Physiol 1991;441:23341.

3. Ashley Z, Sutherland H, Lanmuller H, Russold MF, Unger E, Bijak M, et al. Atrophy, but not necrosis, in rabbit skeletal muscle denervated for periods up to one year Am J Physiol Cell Physiol 2007;292:C440-451.

4. Lanmüller H, Ashley Z, Unger E, et al. Implantable device for long-term electrical stimulation of denervated muscles in rabbits. Med Biol Eng Comput 2005;43:535540

5. Ashley Z, Salmons S, Boncompagni S, et al. Effects of chronic electrical stimulation on long-term denervated muscles of the rabbit hind limb. J Muscle Res Cell Motil 2007;28:203-217.

6. Ashley Z, Sutherland H, Russold MF, et al. Therapeutic stimulation of denervated muscles: the influence of pattern. Muscle Nerve 2008;38:875-886.

$* * * * *$

\section{Computational modeling of $\mathrm{x}$-ray CT cross-sections of thighs of elderly patients}

Paolo Gargiulo (1), Kyle Edmunds (1), Sigurdur Sigurdsson (3), Ugo Carraro (2), Vilmundur Gudnason (3)

(1) Institute for Biomedical and Neural Engineering/ Biomedical Technology Centre Reykjavik University \& Landspitali; (2) Icelandic Heart Association, Reykjavik, Iceland; (3) IRCCS Fondazione Ospedale San Camillo, Venezia, Italy

E-mail: "Paolo Gargiulo" <paologar@landspitali.is>

This work initially began within a study called AGESReykjavik Study, that was initiated to examine genetic susceptibility and gene/environment interaction as these contribute to phenotypes common in old age, a collaborative study between the National Institute on Aging, NIH and the Icelandic Heart Association. ${ }^{1}$ In this frame we are assessing over 3,200 patients from ages 66-93 for changes in muscular and fat content within thigh cross-sections as a function of various measured conditions and pathologies. This wealth of data is historically unique in both its size and variety of explored elderly patient conditions, and discerning how best to analyze both the CT images and patient database could greatly impact modern geriatric medicine and the scientific understanding of aging. We have begun to develop a novel computational method for analyzing each patient's CT contrast histogram by employing analytical methods that allow to create subject specific muscle profiles. ${ }^{2,3}$ These profiles are used to assess and correlate muscle quality with subject co-morbidities. 


\section{CIR-Myo News: Abstracts of the 2015 Spring Padua Muscle Days}

Terme Euganee Padua (Italy), March 12 - 14, 2015

\section{Abstracts}

U.C. thanks the Interdepartmental Research Center of Myology at the Department of Biomedical Sciences, University of Padova, Italy for collaboration and hospitality and the Ludwig Boltzmann Institute of Electrical Stimulation and Physical Rehabilitation of Vienna at the Department of Physical Medicine, Wilhelminenspital, Vienna, Austria for support and collaboration.

1. Harris TB1, Launer LJ, Eiriksdottir G, et al. Age, gene/environment susceptibility - Reykjavik Study: multidisciplinary applied phenomics. Am J Epidemiol 2007;165:1076-87. Epub 2007 Mar 10.

2. Wiedemann L, Chaberova J, Edmunds KJ, et al. Lowamplitude craniofacial EMG power spectral density and 3D muscle reconstruction from MRI. Eur J Transl Myol Basic Appl Myol 2015;25:93-9.

3. Magnússon $\mathrm{B}$, Pétursson $\mathrm{P}$, Edmunds $\mathrm{KJ}$, et al. Improving planning and post-operative assessment for Total Hip Arthroplasty. Eur J Transl Myol - Basic Appl Myol 2015;25:101-8

$$
* * * * *
$$

\section{Muscle hypertrophy with concentric and eccentric training: architectural, molecular, metabolic and functional adaptation}

Martino V Franchi (1), Neil D Reeves (2), Costantinos Maganaris (3), Ken Smith (1), Philip J Atherton (1), Marco V Narici (1)

(1) University of Nottingham, Division of Medical Sciences, School of Medicine, MRC-ARUK Centre for Musculoskeletal Ageing Research, Derby, UK; (2) School of Healthcare Science, Manchester Metropolitan University, Manchester, UK; (3) Research Institute for Sport and Exercise Sciences (RISES), Liverpool John Moores University, UK.

E-mail: "Martino Franchi" <M.Franchi@ nottingham.ac.uk>

Resistance training (RT) is still nowadays considered as the main counter actor of skeletal muscle loss (sarcopenia) and weakness in ageing scenarios. ${ }^{1-3}$ Traditionally, Conventional RT consists in lifting and lowering a constant external load: however, a physiological de-recruitment of motor units (MUs) naturally occurs during the load-lowering, eccentric phase. ${ }^{4}$ A substantial number of works reported considerable strength gains after Conventional RT in older adults, ${ }^{5}$ but only few investigated the muscular adaptations in response to pure concentric vs. pure eccentric RT. As widely known, according to the F-V curve, pure eccentric actions can develop greater forces compared to concentric ones, ${ }^{6}$ and therefore greater training loads could be used. Thus, the general consensus up to date was that eccentric exercise could potentially be preferable over concentric in terms of muscle growth promotion. Lastayo et al $(2003),{ }^{7}$ using backward cycling to maximise eccentric loading (avoiding de-recruitment of MUs), showed the superiority of pure eccentric RT to traditional RT in enhancing muscle mass and strength in older individuals. This apparent superiority of eccentric RT in combating sarcopenia and weakness in old age, prompted a series of investigations by our Lab to explore the mechanical, structural, molecular and metabolic mechanisms of such adaptations. When comparing 14 wk 3 times/week of pure eccentric RT (ECC) vs Conventional RT (CONV RT) in older males aged 65-77 years, both regimes resulted in a similar increase in vastus lateralis (VL) muscle size $(12 \%)$ but with distinctly different VL architectural changes. ${ }^{4}$ The ECC RT promoted a greater increase in VL fascicle length (Lf) (20\%) than CON RT (8\%), while CON RT promoted a greater increase in pennation angle (PA) $(+35 \%)$ than ECC RT (5\%). When investigating the underlying molecular mechanisms of these findings in a following study in young people, ${ }^{8}$ we found morphological adaptations similar to those observed in the older individuals (increase in VL vol of $+6 \%$ with ECC RT and $+8 \%$ with CON RT (pure concentric RT), greater Lf increase with ECC $(12 \%)$ than $\mathrm{CON}(5 \%)$ and greater increase in PA with CON $(30 \%)$ than with ECC RT (5\%). Interestingly, MAPK activation (p38MAPK, ERK1/2, p90RSK) was specific just to ECC RT, while neither mode affected AKT-mTOR or inflammatory signalling $30 \mathrm{~min}$ after exercise. Hence the present findings do not support the belief that ECC loading leads to greater hypertrophy and strength gains than $\mathrm{CON}$ loading, neither in young nor in older individuals. However, muscle hypertrophy is obtained through distinctly different architectural adaptations: while ECC RT appears to stimulate preferential addition of sarcomere in series, CON training seems to promote preferential addition of sarcomere in parallel. The main difference in the hypertrophic responses to CON and ECC RT may actually be not in the amount but then in the location where sarcomere addition occurs (Franchi et al. unpublished)

1. Häkkinen K, Newton RU, Gordon SE, et al. J Gerontol A Changes in muscle morphology, electromyographic activity, and force production characteristics during progressive strength training in young and older men Biol Sci Med Sci 1998;53:B415-23.

2. Narici MV, Maganaris C, Reeves N. Myotendinous alterations and effects of resistive loading in old age. Scand J Med Sci Sports 2005;15,392-401.

3. Seynnes OR, de Boer M, Narici MV. Early skeletal muscle hypertrophy and architectural changes in response to high-intensity resistance training. J Appl Physiol 2007;102,368-73.

4. Reeves ND, Maganaris CN, Longo S, Narici MV. Differential adaptations to eccentric versus conventional resistance training in older humans. Exp Physiol 2009;94:825-33.

5. Macaluso A, De Vito G. Muscle strength, power and adaptations to resistance training in older people. Eur $\mathrm{J}$ Appl Physiol 2004;91:450-72.

6. Katz B. The relation between force and speed in muscular contraction. J Physiol 1939;96:45-64.

7. LaStayo PC, Ewy GA, Pierotti DD, et al. The positive effects of negative work: increased muscle strength and decreased fall risk in a frail elderly population. J Gerontol A Biol Sci Med Sci 2003;58:M419-24.

8. Franchi MV, Atherton PJ, Reeves ND, et al. Architectural, functional and molecular responses to concentric and eccentric loading in human skeletal muscle. Acta Physiol (Oxf) 2014;210:642-54. 


\title{
CIR-Myo News: Abstracts of the 2015 Spring Padua Muscle Days
}

\author{
Terme Euganee Padua (Italy), March 12 - 14, 2015
}

\section{Abstracts}

High resolution micro-CT with iodine contrast in skeletal and cardiac muscle

Robert S Stephenson, Jonathan C Jarvis

Liverpool John Moores University, UK

E-mail: "Jarvis, Jonathan" <J.C.Jarvis@1jmu.ac.uk>

We have used micro-CT imaging with iodine potassium iodide contrast to provide high resolution images of cardiac muscle ${ }^{1,2}$ and skeletal muscle. ${ }^{3}$ The technique is based on differential attenuation between muscle cells and surrounding connective tissues, which in turn is based on the differential uptake of aqueous iodine into the various tissue types. Cardiac myocytes are on average only about 20 microns wide, and so are just at the limit of resolution of this technique, using high resolution scans of sample preparations we are beginning to reveal the much debated micro-anatomy of the complex cardiac mesh. There is considerable interest in the internal 3D structure of cardiac muscle, because the orientation and interweaving of myocyte aggregations determines the functional movement of the walls that provides the remarkable pumping function of the heart. The myocytes do not produce a force against a unique external structure but rather populations work both together and antagonistically to pressurise and to eject the momentarily contained blood volume. The disposition of the myocyte aggregates also influences the temporal sequence of their own rhythmic depolarisation, because conduction is faster in the long axis of the myocytes than in the short axis. In skeletal muscle in which individual fibres are unbranched and cylindrical, it is possible to distinguish individual fibres using micro-CT ${ }^{3}$ In skeletal muscle, the mechanical situation is often thought of as simpler in that fibres act relatively independently to generate a force at their myotendinous junction which acts to draw insertion points towards the fibre origins. However we know that fibre orientation can also be complex in skeletal muscles, particularly in the myotendinous regions. There is also evidence of transverse distribution of force such as illustrated by PA Huijing. ${ }^{4}$ Physical therapists are particularly interested in painful trigger points in muscle that can be identified by ultrasound examination as hypoechoic and with increased resistance to blood flow. ${ }^{5}$ They are often interpreted as areas of fascial tissue that have sub-normal motility, or areas of adhesion, restricting the smooth movement of one muscle relative to another, or one part of a muscle relative to another. Physical therapy in this case aims to improve internal movement. Micro CT of fixed skeletal muscle, along with ultrasound of working muscle will help us to understand the internal as well as the external transmission of force.

1. Stephenson RS, Boyett MR, Hart G, et al. Contrast enhanced micro-computed tomography resolves the 3dimensional morphology of the cardiac conduction system in mammalian hearts. PLoS One 2012;7(4):e35299. doi: 10.1371/journal.pone.0035299. Epub 2012 Apr 11.

2. Jarvis JC, Stephenson R. Studying the microanatomy of the heart in three dimensions: a practical update. Front Pediatr 2013 Oct 10;1:26

3. Jeffery NS, Stephenson RS, Gallagher JA, et al. Microcomputed tomography with iodine staining resolves the arrangement of muscle fibres. J Biomech 2010:40;1.
4. Huijing PA. Epimuscular myofascial force transmission: a historical review and implications for new research. International Society of Biomechanics Muybridge Award Lecture, Taipei, 2007. J Biomech 2009;421:9-21

5. Sikdar S1, Shah JP, Gebreab T, et al. Novel applications of ultrasound technology to visualize and characterize myofascial trigger points and surrounding soft tissue. Arch Phys Med Rehabil 2009;90:1829-38.

$$
* * * * *
$$

\section{Role of radiologic imaging in genetic and acquired neuromuscular disorders}

Paolo Ortolan, Riccardo Zanato, Alessandro Coran, Valeria Beltrame, Roberto Stramare

CIR-Myo, Department of Medicin, Unit of Radiology, University of Padova, Italy

E-mail: roberto.stramare@unipd.it

Great technologic and clinical progress have been made in the last decades in identifying genetic defects of several neuromuscular diseases. However, the diagnosis is usually challenging, due to great variability in genetic abnormalities and clinical phenotypes, the complexity of the molecular genetic approaches and the poor specificity of complementary analyses. Muscle biopsy represents the gold standard for the diagnosis of genetic neuromuscular diseases, clinical imaging of muscle tissue is an important diagnostic tool to identify and quantifies muscle changes. Radiologic imaging is, indeed, increasingly used as a diagnostic tool to describe patterns and extent of muscle involvement. ${ }^{1-5}$ Computer tomography (CT) and ultrasound (US) due to some of their shortcomings have given way in the diagnosis of neuromuscular diseases to Magnetic resonance imaging (MRI), which is a technique that doesn't use ionizing radiation, has a higher contrast resolution and allows early recognition of the initial phases of inflammation and dystrophies fatty degeneration of some early myopathies. In conclusion, radiologic imaging is increasingly playing a relevant role in neuromuscular disorders, in particular in those of genetic etiology that are difficult to be characterized.

1. Quijano-Roy S, Avila-Smirnow D, Carlier RY, WB-MRI muscle study group. Whole body muscle MRI protocol: Pattern recognition in early onset NM disorders. Neuromuscul Disord 2012;22 Suppl 2:S68-84.

2. Wattjes MP, Kley RA, Fischer D. Neuromuscular imaging in inherited muscle diseases. Eur Radiol 2010;20:2447-60.

3. Stramare R, Beltrame V, Dal Borgo R, et al: MRI in the assessment of muscular pathology: a comparison between limb-girdle muscular dystrophies, hyaline body myopathies and myotonic dystrophies. Radiol Med 2010;115(4):585-99. doi: 10.1007/s11547-010-0531-2. Epub 2010 Feb 22. English, Italian.

4. Gargiulo P, Reynisson PJ, Helgason B, et al. Muscle, tendons, and bone: structural changes during denervation and FES treatment. Neurol Res 2011;33:750-8. doi: 10.1179/1743132811Y.0000000007.

5. Ortolan P, Zanato R, Coran A, et al. Role of radiologic imaging in genetic and acquired neuromuscular disorders. Eur J Transl Myol/Basic Appl Myol 2015;25, in press 


\title{
CIR-Myo News: Abstracts of the 2015 Spring Padua Muscle Days
}

\author{
Terme Euganee Padua (Italy), March 12 - 14, 2015
}

\section{Abstracts}

\section{Proteomics of single skeletal muscle fibers: focusing on structural and metabolic diversity}

Marta Murgia (1,2), Nagarjuna Nagaraj (1), Atul Deshmukh (1), Marlis Zeiler (1), Pasqua Cancellara (2), Carlo Reggiani (2), Stefano Schiaffino (3) and Matthias Mann (1)

(1) Department of Proteomics and Signal Transduction, MaxPlanck-Institute of Biochemistry, Martinsried, Germany; (2) Department of Biomedical Sciences, University of Padova, Italy; (3) Venetian Institute of Molecular Medicine, Padua, Italy

E-mail: "Murgia, Marta" <mmurgia@ biochem.mpg.de>

Muscle fibers are multinucleated single cells originating from myoblasts fusion which, under the control of developmental cues, develop distinct molecular composition and physiological properties. The four basic fiber types (slow type1, and fast type $2 \mathrm{~A}, 2 \mathrm{X}$ and $2 \mathrm{~B}$ ) vary greatly in their contractile and metabolic properties and are identified based on the molecular properties of the corresponding myosin heavy chain isoforms. ${ }^{1}$ Interestingly, primary muscle diseases and metabolic disorders often affect, or spare, specific fiber types. Despite recent advances in mass spectrometry (MS)based proteomics, ${ }^{2,3}$ single cells have been beyond reach so far, preventing fiber type-resolved studies in skeletal muscle. Although large compared to average mononuclear cells, muscle fibers contain relatively limited protein amounts. They have a highly unfavorable dynamic range dominated by highly abundant sarcomeric proteins, which has also limited proteomic studies at the whole muscle level. Furthermore, a muscle has a heterogeneous composition, including not only muscle fibers but also connective and adipose tissue, blood vessels and nerves, with possible variations of their relative proportions in pathophysiological conditions. With these challenges in mind, we set out to develop a high-sensitivity proteomics workflow that has allowed us to obtain the proteome of single mouse muscle fibers. We could also analyse and compare the proteomes of different segments of the same fiber. Our strategy allowed unbiased fiber-type assignment, leading to the discovery of novel type-specific features. Our results show fiber type-specific patterns of mitochondrial specialization, revealing alternative utilization of metabolic intermediates that fine-tune fiber types to their tasks. ${ }^{4}$ As the single cell proteomics analysis performed here is rapid and robust, it can be applied to a wide variety of physiological and pathological conditions.

1. Schiaffino S, Reggiani C (2011) Fiber types in mammalian skeletal muscles. Physiol Rev 91: 14471531.

2. Kulak NA, Pichler G, Paron I, Nagaraj N, and Mann M (2014). Minimal, encapsulated proteomic-sample processing applied to copy-number estimation in eukaryotic cells. Nat Methods 11: 319-324.

3. Cox J, Mann M (2008) MaxQuant enables high peptide identification rates, individualized p.p.b.-range mass accuracies and proteome-wide protein quantification. Nat Biotechnol 26: 1367-1372.

4. Murgia M Nagaraj N, Deshmukh AS, et al. (2015) Single muscle fiber proteomics reveals unexpected mitochondrial specialization. EMBO Rep 2015 Feb 2. pii: e20143975. [Epub ahead of print].
Functional electrical stimulation (FES) use in horses for musculoskeletal and neuromuscular rehabilitation

Sheila J. Schils

Equine Rehabilitation, EquiNew, LLC, River Falls, Wisconsin, United States

E-mail: sbSchils@EquiNew.com

Functional electrical stimulation (FES) has been used for over 15 years in equine rehabilitation and hundreds of case studies show the positive clinical outcomes of FES to reduce equine muscle spasms. ${ }^{1}$ FES is the most applicable electrotherapy for equine rehabilitation due to the ability of the device to stimulate deep enough to reach the skeletal support muscles of the horse while obtaining a high compliance. $^{2}$ FES is also used for early mobilization after injury or surgery to obtain controlled, precise movement while the horse is confined. ${ }^{3}$ In addition FES has been used as a treatment for recurrent laryngeal neuropathy in horses. ${ }^{4}$ The goals of FES treatments for use in equine rehabilitation is to: 1. Reduce muscle spasms therefore reduce pain 2 . Produce symmetrical muscle movement to improve skeletal alignment 3. Stimulate deep skeletal support muscles 4 . Reeducate muscle memory to improve movement patterns 5 . Strengthen muscle 6 . Reduce muscle atrophy and 7 . Recruit fast and slow twitch muscle fibers in all stages of rehabilitation. The fundamental purpose of the FES treatments in horses is to improve functional movement so that the horses are more comfortable and balanced, and therefore can perform better and have fewer chances of injury due to improved mechanics. The FES system used on horses has specific characteristics that may or may not be found in human FES systems. The signal must be produced by a microcontroller so that the clarity and control of the signal is precise. The stimulus must be able to reach comfortably $20 \mathrm{~cm}$ deep to activate the deep core muscles of the horse. The system must be portable and must be able to be run off a battery so that no external source of electricity is needed. The FES system for horses weighs about 2 pounds and attaches to a surcingle, which is strapped around the thorax of the horse. The waveform is rectangular, with a zero net charge and the pulse duration is 250 microseconds positive/negative. Carbon electrodes are placed in a pad about $55 \mathrm{~cm}$ long, which is used for treatments to the axial skeleton. Self-stick electrodes are placed on the neck and are also used for other site-specific applications. The typical voltage to produce strong muscle contractions, resulting in functional movement, along the top line of the horse and on the neck is approximately 7-9 volts. Treatment time varies from 20-35 minutes. Typically, 2 adjacent sites are treated on the same day, for example, the neck and thorax. A clinical change in the reduction of the severity of muscle spasms usually occurs after 2-4 FES treatments. Positive results utilizing this FES system and protocols have been obtained by a variety of veterinarians and equine physiotherapists. Functional electrical stimulation has produced encouraging rehabilitation outcomes in horses, however it is not extensively utilized in veterinary medicine. Equine case studies have shown examples of the use of FES to reverse muscle atrophy and decrease muscle spasticity. Exploring the application and outcomes of the use of FES for rehabilitation 


\title{
CIR-Myo News: Abstracts of the 2015 Spring Padua Muscle Days
}

\author{
Terme Euganee Padua (Italy), March 12 - 14, 2015
}

\section{Abstracts}

in horses may provide some interesting information for the utilization of FES in human rehabilitation.

1. Schils SJ, Turner TA. Functional Electrical Stimulation for equine epaxial muscle spasms:retrospective study of 241 clinical cases. Comparative Exercise Physiology. 2014;10:89-97.

2. Schils SJ. Review of Electrotherapy Devices for Use in Veterinary Medicine. Proceedings 55th Annual AAEP Convention, Las Vegas, Dec 5-9, 2009 p. 68-73.

3. Schils SJ, Turner TA. Review of Early Mobilization of Muscle, Tendon, and Ligament After Injury in Equine Rehabilitation. Proceedings 56th Annual AAEP Convention, Baltimore, Dec 4-8, 2010 p. 374-380

4. Cheetham J, Regner A, Jarvis JC, et al. Functional electrical stimulation of intrinsic laryngeal muscles under varying loads in exercising horses. PLoS One. 2011 6: e24258.

\section{Mitochondrial density and distribution by histochemical approaches distinguish muscle fiber types and support clinical improvements due to FES as a treatment of equine epaxial muscle spasms}

Barbara Ravara (1), Valerio Gobbo (2), Ugo Carraro (3), Lin Gelbmann (4), Jamie Pribyl (5), Sheila Schils (6)

(1) Laboratory of Translational Myology of the Interdepartmental Research Center of Myology, Department of Biomedical Science, University of Padova, Italy; (2) C.N.R. Institute of Neuroscience, Department of Biomedical Science, University of Padova, Italy; (3) IRRCS Fondazione Ospedale San Camillo, Venezia, Italy; (4) LIN Rehabilitation Services LLC, St Paul, Minnesota USA; (5) Jamie Pribyl, DVM, Buffalo, Minnesota USA; (6) Equine Rehabilitation LLC, River Falls, Wisconsin USA

E-mail: barbara.ravara@unipd.it

Functional Electrical Stimulation (FES) has been used extensively over several decades to reverse muscle atrophy during rehabilitation for spinal cord injury patients. ${ }^{1-3}$ The benefits of the technology are being expanded into other areas, and FES has been recently utilized for injury rehabilitation and performance enhancement in horses. ${ }^{4}$ FES can obtain precise, controlled functional movement and therefore can be used to initiate conservative movement early in the rehabilitation plan, as well as obtain more aggressive movement during the later stages of healing. Six retired horses, that had been previously used mainly for dressage riding, were selected for this study. The horses ranged in age from 10 to $17 \mathrm{yr}$ and had all been clinically evaluated by veterinarians for axial musculoskeletal skeletal pathologies and none had been noted. Clinical evaluation found epaxial muscle spasms in all horses with minimal to no pelvic extension when manually palpated. FES treatments were performed on the sacral/lumbar region 3 times per week for a period of 8 weeks. The Modified Ashworth Scale for grading muscle spasms found a one grade improvement after approximately 4 FES treatments, indicating improved functional movement of the sacral/lumbar region, supporting the evidence by clinical palpations that a reduction in epaxial muscle spasms occurred. Skeletal muscle biopsies Pre and Post FES treatments were obtained from the longissimus lumborum muscle at a depth of $3 \mathrm{~cm}$ on the same side of each horse. Cryosections were stained with a HemotoxylinEosin (H-E), and nicotinamide adenine dinucleotide tetrazolium reductase reaction (NADH-TR). The eventual size change of the muscle fibers due to FES or co-morbidities were evaluated by morphometry in H-E and NADH-TR stained cryosections, while in the NADH-TR slides the density and distribution of mitochondria were also determined. ${ }^{5}$ Main results of the morphometric analyses were: 1) As expected for the type of FES treatment used in this study, only a couple of horses showed significant increases in mean muscle fiber size when Pre- vs Post-FES biopsies were compared; 2) In the older horses, there were sparse (or several in one horse) severely atrophic and angulated muscle fibers in both Pre- and Post-FES samples, whose distribution suggests they were denervated due to a distal neuropathy; 3) The hypothesis of generalized FESinduced muscle fiber damage during epaxial muscle training is not supported by our data since: 3.1) Denervated muscle fibers were present in the Pre-FES biopsies and 3.2) Only one horse (age $15 \mathrm{yr}$ ) presented with high numbers of longterm denervated muscles fibers Post-FES; 4) Preliminary data indicate that the increased density and distribution of mitochondria in Post-FES biopsies suggests that the clinical improvements in the treated horses may be related to the increased muscle contractions, therefore improving muscle perfusion which is induced by FES training. In conclusion, FES in horses is a safe treatment that provides clinical improvements in equine epaxial muscle spasms.

U.C. thanks the Interdepartmental Research Center of Myology at the Department of Biomedical Sciences, University of Padova, Italy for collaboration and hospitality and the Ludwig Boltzmann Institute of Electrical Stimulation and Physical Rehabilitation of Vienna at the Department of Physical Medicine, Wilhelminenspital, Vienna, Austria for support and collaboration.

1. Kapadia N, Masani K, Catharine Craven B, et al. A randomized trial of functional electrical stimulation for walking in incomplete spinal cord injury: Effects on walking competency. J Spinal Cord Med 2014;37:51124. doi: 10.1179/2045772314Y.0000000263.

2. Kern H, Carraro U, Adami N, et al. Home-based functional electrical stimulation rescues permanently denervated muscles in paraplegic patients with complete lower motor neuron lesion. Neurorehabil Neural Repair. 2010;24:709-21. doi: 10.1177/1545968310366129. Epub 2010 May 11.

3. Kern H, Carraro U. Home-based Functional Electrical Stimulation (h-b FES) for long-term denervated human muscle: History, basics, results and perspectives of the Vienna Rehabilitation Strategy. Eur J Transl Myol/Basic Appl Myol 2014:24:27-40. DOI: 10.4081/bam.2014.1.27.

4. Schils SJ, Turner TA. Functional Electrical Stimulation for equine epaxial muscle spasms:retrospective study of 241 clinical cases. Comparative Exercise Physiology, 2014;10: 89-97.

5. Ravara B, Gobbo V, Carraro U, et al. Functional electrical stimulation as a safe and effective treatment for equine epaxial muscle spasms: Clinical evaluations and histochemical morphometry of mitochondria in muscle biopsies. Eur J Transl Myol 2015;25:109-20. 


\title{
CIR-Myo News: Abstracts of the 2015 Spring Padua Muscle Days
}

\author{
Terme Euganee Padua (Italy), March 12 - 14, 2015
}

\section{Abstracts}

\section{From RISE to SAVe: technical concepts for delay of ventilator dependency in ALS}

\author{
Winfried Mayr (1), Matthias Krenn (1), Ugo Carraro (2)
}

(1) Centre for Medical Physics and Biomedical Engineering, Medical University of Vienna, Austria; (2) (2) IRCCS Fondazione Ospedale San Camillo, Venezia, Italy

E-mail: winfried.mayr@meduniwien.ac.at

FES in neuromuscular dystrophy diseases has been a controversial topic through the past decades, generally associated with the concern, that voluntary or stimulated activation of nerves and muscles can lead to metabolic overuse and acceleration of degenerative processes. ${ }^{1}$ More recent literature on clinical treatments with FES with vastly different parameters has found strong hints for beneficial influence and no real indications for adverse effects. In the particular case of ALS with progressive denervation of motor units, rapid loss of muscular functions and inevitable development towards respiratory insufficiency threshold and dependence on artificial respiration, usually in intensive care environment. A recent development of a stimulator with percutaneous electrodes, which can activate still innervated motor units of the diaphragm and train size, contraction force and endurance of their muscle fibers to compensate part of the denervation induced capacity loss. ${ }^{2}$ It has been shown in studies, that respirator dependence can be delayed from in average 40 month after disease onset, by about 16 months. After the European project RISE has shown that denervated muscle fibers can be maintained, trained and functionally activated by non-invasive application of long-duration stimuli, ${ }^{3-6}$ and earlier studies have demonstrated, that similar results can be achieved with implantable electrodes, there is a high probability, that in case of ALS already denervated muscle fibers can be activated and maintained with similar approaches, which could lead to further delays in respirator dependence.

1. Wijesekera LC, Leigh NP. Amyotrophic lateral sclerosis. Orphanet J Rare Dis. 2009; 4:3.

2. http://www.synapsebiomedical.com/als/neurx-als.shtml

3. Mayr W, Hofer C, Bijak M, et al. Functional electrical stimulation (FES) of denervated muscles: existing and prospective technological solutions. Basic Appl Myol 2002;12):287-90.

4. Kern H, Carraro U, Adami N, et al. Home-based functional electrical stimulation rescues permanently denervated muscles in paraplegic patients with complete lower motor neuron lesion Neurorehabil Neural Repair. 2010; 24:709-721.

5. Kern H, Carraro U, Adami N, et al. One year of homebased daily FES in complete lower motor neuron paraplegia: recovery of tetanic contractility drives the structural improvements of denervated muscle. Neurol Res 2010; 32: 5-12.

6. Kern H, Carraro U. Home-based Functional Electrical Stimulation (h-b FES) for long-term denervated human muscle: History, basics, results and perspectives of the Vienna Rehabilitation Strategy. Eur J Transl Myol/Basic Appl Myol 2014:24:27-40. DOI: 10.4081/bam.2014.1.27.
Respiratory insufficiency in ALS - present solutions for symptom alleviation and/or prolonging the lifespan

\author{
Janez Zidar
}

Institute of Clinical Neurophysiology, University Medical Centre Ljubljana, Ljubljana, Slovenia

E-mail: janez.zidar@kclj.si

Respiratory failure is the most common cause of death in ALS. Early symptoms of respiratory insufficiency are diverse and most commonly occur during sleep. They may severely impede the patients' quality of life. It is important that respiratory dysfunction is recognized and discussed with patients early enough. Several methods are in use to alleviate such symptoms or even prolong life of these patients. Noninvasive intermittent ventilation (NIV) is primarily aimed at lessening symptoms severity rather than prolonging life. It improves the quality of sleep and of cognitive function, and relieves morning headaches. The alternative is invasive ventilation through tracheostomy which prolongs life but does not affect disease progression. It also precludes the patients' ability of oral communication. They greatly increase care needs and, consequently, deteriorate quality of life, at least that of the carers. The search for new approaches to treatment of respiratory failure has led to the development of diaphragm pacing, that is currently an approved method only in the USA. However, there are important unanswered questions regarding its benefit and impact. It may only improve patients' quality of life, with better sleep and daytime functioning, better breathing, and less fatigue but it does not prolong life. A small number of patients decide not to use any of the above methods while others, mostly those with severe bulbar involvement, do not tolerate NIV. In them, the feeling of shortness of breath can be reduced by the administration of morphine. There are substantial differences in the use of the above mentioned methods in different countries especially in the use of mechanical ventilation via tracheostomy. ${ }^{1-5}$ We recently analysed data of 271 ALS patients treated at the Institute of Clinical Neurophysiology in the 10-year period between 2003 and 2012. Their mean age at symptoms onset was $62.7 \pm 11.4$ years, and mean survival from the time of enrolment $16.4 \pm 15.1$ months. One hundred seventy nine $(66.1 \%)$ patients had spinal onset and $71(26.2 \%)$ bulbar onset of the disease. In total $34.7 \%$ of all patients consented to the non-invasive assisted ventilation at some point. The proportion of those using non-invasive respiratory support was rising through the analysed years and has reached over $50 \%$, reported also by other tertiary care centres. Survival after institution of non-invasive ventilation was $7.8 \pm 7.3$ months. Six patients used invasive ventilation, two of them started using it before the diagnosis was established. This is less than in some other countries. Four patients had a tracheostomy done solely for the airway hygiene. Diaphragm pacing is currently not in use by the Ljubljana ALS team.

1. Bourke SC, Bullock RE, Williams TL, et al. Noninvasive ventilation in ALS: indications and effect on quality of life. Neurology 2003;61:171-7.

2. Chiò A, Calvo A, Ghiglione $\mathrm{P}$, et al. Tracheostomy in amyotrophic lateral sclerosis: a 10-year population-based study in Italy. J Neurol Neurosurg Psychiatry 2010;81:1141-3. 


\section{CIR-Myo News: Abstracts of the 2015 Spring Padua Muscle Days}

Terme Euganee Padua (Italy), March 12 - 14, 2015

\section{Abstracts}

3. Chiò A, Calvo A, Moglia C, et al. Non-invasive ventilation in amyotrophic lateral sclerosis: a 10 year population based study. J Neurol Neurosurg Psychiatry 2012;83:377-81.

4. Leonardis L, Dolenc Goršelj L, Vidmar G. Factors related to respiration influencing survival and respiratory function in patients with amyotrophic lateral sclerosis: a retrospective study. Eur J Neurol 2012;19:1518-24.

5. Miller RG, Jackson CE, Kasarskis EJ, et al. Practice Parameter update: The care of the patient with amyotrophic lateral sclerosis: Multidisciplinary care, symptom management, and cognitive/behavioral impairment (an evidence-based review): Report of the Quality Standards Subcommittee of the American Academy of Neurology. Neurology 2009;73:1227-33.

$$
* * * * *
$$

\section{Structural and functional characteristics of denervated muscles from oldest-old rats: a relevant animal model for FES of denervated myofibers of the diaphragm in ALS?}

Simone Mosole $(1,2)$, Sandra Zampieri $(1,2)$, Elena Germinario (3), Valerio Gobbo (4), Barbara Ravara (1,2), Daniela Danieli-Betto (3), Ugo Carraro (5)

(1) Translational Myology Lab of the Interdepartmental Research Center of Myology, Department of Biomedical Science, University of Padova, Italy; (2) Ludwig Boltzmann Institute of Electrical Stimulation and Physical Rehabilitation, Wilhelminenspital Wien, Austria; (3) Interdepartmental Research Center of Myology, Department of Biomedical Science, University of Padova, Italy; (4) C.N.R. Institute of Neuroscience, Department of Biomedical Science, University of Padova, Italy; (5) IRCCS Fondazione Ospedale San Camillo, Venezia, Italy

E-mail: ugo.carraro@unipd.it

In managements of ALS, a syndrome that is very often fatal due to a ventilation crisis, palliative therapies are only possible, at least for the predictable future. ${ }^{1}$ On the other hand, we are confident we may extend function of respiratory muscles, and thus postponing the need of air pumping that damages even more the diaphragm muscle, ${ }^{2}$ if we will be able to show in experimental models that some additional muscle contractile function is achievable by combining proven approaches to maintain/recover contractility of "denervated" muscle fibers of the diaphragm in animal models of ALS. ${ }^{3}$ The left hemi-diaphragm of 3-and 30-month old rats (adult and oldest old, respectively) was denervated under general anesthesia by section of the left phrenic nerve, reached through an intercostal approach. The left sciatic nerve was also severed. Rats were sacrificed after 7 days. Isometric mechanical measurements were performed in vitro in a vertical muscle apparatus $(300 \mathrm{~B}$, Aurora Scientific Inc, Canada) containing a Ringer solution of the following composition: $120 \mathrm{mM} \mathrm{NaCl}, 4.7 \mathrm{mM} \mathrm{KCl}, 2.5 \mathrm{mM} \mathrm{CaCl}_{2}$, $3.15 \mathrm{mM} \mathrm{MgCl}_{2}, 1.3 \mathrm{mM} \mathrm{NaH} \mathrm{PO}_{4}, 25 \mathrm{mM} \mathrm{NaHCO}{ }_{3}, 11$ $\mathrm{mM}$ glucose, $30 \mu \mathrm{M}$ d-tubocurarine, $\mathrm{pH} 7.2-7.4,30^{\circ} \mathrm{C}$, bubbled with $95 \% \mathrm{O}_{2}-5 \% \mathrm{CO}_{2}$. Two strips from each hemidiaphragm were examined. Each strip was stretched to the optimal length (i.e. the length that allowed maximal tension development in response to a single pulse) and electrically stimulated, by two parallel electrodes, with supramaximal pulses ( $0.5 \mathrm{~ms}$ duration) delivered by a Grass S44 electronic stimulator through a stimulus isolation unit (Grass SIU5). Muscle response was recorded through an isometric force transducer (Harvard) connected to an AT-MIO 16AD acquisition card (National Instruments) and data were analyzed by a specific module of the National Instruments Labview software. Force-frequency curve was determined by stimulating the muscle at $1,15,30,60,80,120$ and $150 \mathrm{~Hz}$. All tensions were normalized to the muscle wet weight (specific tension, $\mathrm{N} \mathrm{g} \mathrm{g}^{-1}$ ). Contraction and relaxation times, and twitch and tetanic tensions were similar in adult and old innervated diaphragm. Denervation caused similar slowing of contractile properties in adult and old muscles. Interestingly, denervated diaphragm produced a significant higher specific tension with respect to the contralateral innervated up to 15 $\mathrm{Hz}$ stimulation in adult but not in old muscles. Instead, a similar drop of tension was evident at higher frequency both in adult and oldest old denervated diaphragm, compared to innervated one. In conculsion contractile properties of diaphragm were not weakened by ageing. On the other hand, mitochondrial density and distribution determined by electron microscopy and histochemistry were substantially changed by age and even more by 7 day denervation, confirming that the muscles were denervated seven days after neurectomy. We are confident, that this animal model, i.e., muscle denervation in oldest-old animals, will be useful in testing cellular (injecting myogenic stem cells derived from adipose mesenchymal cells and physical (FES) approaches. The first step of a long way will be to test if mitochondrial density and distribution may recover to young rat levels by neuromodulation of the hemidenervated diaphragm in oldestold rats.

U.C. thanks the Interdepartmental Research Center of Myology at the Department of Biomedical Sciences, University of Padova, Italy for collaboration and hospitality and the Ludwig Boltzmann Institute of Electrical Stimulation and Physical Rehabilitation of Vienna at the Department of Physical Medicine, Wilhelminenspital, Vienna, Austria for support and collaboration.

1. Wijesekera LC, Leigh NP. Amyotrophic lateral sclerosis. Orphanet J Rare Dis. 2009; 4:3.

2. http://www.synapsebiomedical.com/als/neurx-als.shtml

3. Kern H, Carraro U. Home-based Functional Electrical Stimulation (h-b FES) for long-term denervated human muscle: History, basics, results and perspectives of the Vienna Rehabilitation Strategy. Eur J Transl Myol/Basic Appl Myol 2014:24:27-40. doi: 10.4081/bam.2014.1.27.

\section{Delivering myogenic cells in diseased muscles}

\section{Martina Piccoli, Chiara Franzin, Enrica Bertin, Paolo De Coppi, Michela Pozzobon and co workers}

Stem Cells and Regenerative Medicine Lab, Foundation Institute of Pediatric Research Città della Speranza, Padova, Italy

E-mail: michela.pozzobon@gmail.com

Muscle dysfunction and degeneration of skeletal muscle lead to a perturbation of the regenerative process, causing the 


\title{
CIR-Myo News: Abstracts of the 2015 Spring Padua Muscle Days
}

\author{
Terme Euganee Padua (Italy), March 12 - 14, 2015
}

\section{Abstracts}

premature exhaustion of satellite cell reservoir due to continuous cycles of degeneration/regeneration. The in vivo approach in animal models can be helpful to study the mechanism underneath the operating principle of the stem cell reservoir, namely the niche, which holds great potential to understand the onset of muscle pathologies. To this end, the success of skeletal muscle reconstruction depends on finding the most effective, clinically suitable strategy to repair the damaged tissue. $\mathrm{We}^{1}$ designed and developed the delivery of either SCs or muscle progenitor cells (MPCs) via an in situ photo-cross-linkable hyaluronan-based hydrogel, hyaluronic acid-photoinitiator (HA-PI) complex in a mouse model of muscle mass depletion and more recently, ${ }^{2}$ we systemically injected amniotic fluid stem cells in a mouse model of Spinal Muscle Atrophy. Through these two approaches muscle reconstruction and rejuvenation was associated with the formation of neural and vascular networks in the first model and the reconstitution of a functional SC niche in the second.

1. Rossi CA, Flaibani M, Blaauw B; et al. In vivo tissue engineering of functional skeletal muscle by freshly isolated satellite cells embedded in a photopolymerizable hydrogel. FASEB J 2011;25:2296-304. doi: 10.1096/fj.10-174755. Epub 2011 Mar 30.

2. Piccoli M, Franzin C, Bertin E, et al. Amniotic fluid stem cells restore the muscle cell niche in a HSA-Cre, Smn(F7/F7) mouse model. Stem Cells. 2012;30:1675-84. doi: $10.1002 /$ stem. 1134 .

$$
* * * * *
$$

\section{Persistent muscle fiber regeneration in human long term denervation. Past, present, future}

Ugo Carraro (1), Simona Boncompagni (2), Valerio Gobbo (3), Sandra Zampieri (4,5), Simone Mosole $(4,5)$, Andrea Marcante (1), Francesco Piccione (1), Stefano Masiero (6), Vincenzo Vindigni (7), Paolo Gargiulo (8), Feliciano Protasi (2), Amber Pond (9), Helmut Kern $(5,10)$

(1) IRCCS Fondazione Ospedale San Camillo, Venezia, Italy; (2) CeSI, Center for Research on Aging, Department of Neuroscience, Imaging and Clinical Sciences, University G. d'Annunzio of Chieti, Italy; (3) C.N.R. Institute of Neuroscience, Department of Biomedical Science, University of Padova, Italy; (4) Translational Myology, Interdepartmental Research Center of Myology of the University of Padova (CIR-Myo), Department of Biomedical Science, Padova, Italy; (5) Ludwig Boltzmann Institute of Electrical Stimulation and Physical Rehabilitation, Vienna, Austria; (6) CIR-Myo, Department of Neuroscience, Rehabilitation Unit, University of Padova, Italy; (7) CIRMyo, Department of Neuroscience, Plastic Surgery Unit, University of Padova, Italy; (8) Department of Science, Education, Innovation, Landspitali University Hospital, Reykjavik, Iceland; (9) Anatomy Department, Southern Illinois University, School of Medicine, Carbondale, Illinois, USA; (10) Department of Physical Medicine, Wilhelminenspital, Vienna, Austria.

E-mail: ugo.carraro@unipd.it

Despite the the severely destructive effects of long term denervation there is structural and ultrastructural evidence for survival of muscle fibers in mammals, some surviving at least ten months in rodents and 3-6 years in humans. ${ }^{1}$ Further, in rodents there is evidence that muscle fibers may regenerate even after repeated damage to aneurally regenerated muscle, ${ }^{2}$ this potential being maintained for several months after aneural regeneration. ${ }^{3}$ While permanently denervated human muscle sooner or later loses the ability to contract, even many months after denervation the muscles may almost maintain or recover their size and ability to function if electrically stimulated by long impulses.4 During the past decade, we have studied muscle biopsies from the quadriceps muscle of Spinal Cord Injury (SCI) patients suffering with Conus and Cauda Equina syndrome, a condition that fully and irreversibly disconnects skeletal muscle fibers from their damaged innervating motor neurons. We have indeed demonstrated that human denervated muscle fibers survive surprisingly years of denervation $^{5,6}$ and can be rescued from severe atrophy by home-based Functional Electrical Stimulation (h-bFES). ${ }^{5-8}$ After permanently denervated human muscles reach the minimal $10 \%$ residual volume/weight, we discovered that many severely atrophic muscle fibers still persist, but they present peculiar clusters of centrally located myonuclei. ${ }^{6}$ This features are seemingly the result of complete loss of contractile structures in both fast and slow types muscle fibers and of the redistribution of myonuclei from their spiral subsarcolemmal distribution to groups of central nuclei separated by long stretches of amyofibrillar sarcoplasm. ${ }^{5}$ These peculiarly severe atrophic muscle fibers are present in rodent muscles from seven-months after neurectomy and in human muscles between 30 and 70 months after a spinal cord injury that results in a permanent complete Conus and Cauda Equina Syndrome. ${ }^{5,7}$ These severe atrophic muscle fibers are therefore structurally distinct from early myotubes, one of the early stages of myogenesis during both development and regeneration of adult muscle fibers after necrosis. Whether in humans this is a result of persistent de novo formation of muscle fibers is an open issue we explored using immunohistochemistry in both non-stimulated and h-b FES stimulated human muscles. ${ }^{1,4,5,7,8}$ We have indeed observed the persistent presence of muscle fibers which are positive to labeling by an antibody which specifically recognizes the embryonic myosin heavy chain (MHCemb). Relative to the total number of fibers present, only a small percentage of these MHCemb positive fibers are detected, suggesting that they are regenerating muscle fibers and not pre-existing myofibers re-expressing embryonic isoforms). ${ }^{1,4,5,7,8}$ Although embryonic isoforms of acetylcholine receptors are known to be re-expressed and to spread from the end-plate to the sarcolemma of all muscle fibers in early phases of muscle denervation, we suggest that the MHCemb positive muscle fibers resulted from the activation, proliferation and fusion of satellite cells, the myogenic precursors present under the basal lamina of the muscle fibers. ${ }^{9}$ Beyond reviewing evidence from rodent and human studies, we add some ultrastructural evidence of muscle fiber regeneration in longterm denervated human muscles (i.e., fusing myoblasts, myotubes and the presence of muscle fibers with a double layer of basal lamina) and discuss the options to substantially increase the regenerative potential of long term denervated human muscles not having been treated in time with h-b FES. ${ }^{1,10}$ Some of the mandatory procedures, are indeed ready 


\title{
CIR-Myo News: Abstracts of the 2015 Spring Padua Muscle Days
}

\author{
Terme Euganee Padua (Italy), March 12 - 14, 2015
}

\section{Abstracts}

to be translated from animal experiments to clinical studies to meet the needs of persons with long-term irreversible muscle denervation. ${ }^{10}$ An European Project, the trial Rise4EU (Rise for You, a personalized treatment for recovery of function of denervated muscle in long-term stable SCI) will hopefully follow.

U.C. thanks the Interdepartmental Research Center of Myology at the Department of Biomedical Sciences, University of Padova, Italy for collaboration and hospitality and the Ludwig Boltzmann Institute of Electrical Stimulation and Physical Rehabilitation of Vienna at the Department of Physical Medicine, Wilhelminenspital, Vienna, Austria for support and collaboration.

1. Carraro U, Boncompagni S, Gobbo V, et al. Persistent muscle fiber regeneration in long term denervation. Past, present, future. Eur J Transl Myol - Basic Appl Myol 2015;25:77-92.

2. Mussini I, Favaro G, Carraro U. Maturation, dystrophic changes and the continuous production of fibers in skeletal muscle regenerating in the absence of nerve. $\mathrm{J}$ Neurophatol Exp Neurol 1987;46:315-31.

3. Rossini K, Zanin ME, Carraro U. To stage and quantify regenerative myogenesis in human long-term permanent denervated muscle. Basic Appl Myol 2002;12:277-86.

4. Kern H, Boncompagni S, Rossini K, et al. Long-term denervation in humans causes degeneration of both contractile and excitation contraction coupling apparatus, wich is reversible by functional electrical stimulation (FES). A role for myofiber regeneration? J Neuropathol Exp Neurol 2004;63:919-31.

5. Kern H, Carraro U, Biral D, et al. Severely atrophic muscle fibers with nuclear clumps survive many years in permanently denervated human muscle. The Open Pathology Journal 2009;3:106-10.

6. Boncompagni S, Kern H, Rossini K, et al. Structural differentiation of skeletal muscle fibers in the absence of innervation in humans. Proc Natl Acad Sci USA 2007;104:19339-44.

7. Kern $\mathrm{H}$, Carraro $\mathrm{U}$, Adami $\mathrm{N}$, et al. Home-based functional electrical stimulation rescues permanently denervated muscles in paraplegic patients with complete lower motor neuron lesion. Neurorehabil Neural Repair. 2010;24:709-21. doi: 10.1177/1545968310366129.

8. Carraro U, Rossini K, Mayr W, Kern H. Muscle fiber regeneration in human permanent lower motoneuron denervation: relevance to safety and effectiveness of FES-training, which induces muscle recovery in SCI subjects. Artif Organs 2005;29:187-91.

9. Yablonka-Reuveni Z. The skeletal muscle satellite cell: still young and fascinating at 50. J Histochem Cytochem 2011;59:1041-59. doi: 10.1369/0022155411426780.

1o. Huang H, Sun T, Chen L, et al. Consensus of clinical neurorestorative progresses in patients with complete chronic spinal cord injury. Cell Transplant 2014;23 1:517. doi: 10.3727/096368914X684952. Epub 2014 Oct 9.

\section{An update on treatment for Duchenne Muscular Dystrophy}

Ros Quinlivan

Centre for Neuromuscular Diseases, National Hospital for Neurology and Neurosurgery, London, UK

E-mail: "Quinlivan, Rosaline" <r.quinlivan@ucl.ac.uk>

Duchenne Muscular Dystrophy (DMD) is the most common childhood onset dystrophy affecting 1:3,500-6000 live male births. It is a rapidly progressive muscle wasting condition which, without treatment, causes death in teenage years due to cardiac and respiratory failure. Since the 1990s survival has improved due to the introduction of non-invasive nighttime home ventilation and early and aggressive treatment of cardiomyopathy using with ACE inhibitors and beta blockers. ${ }^{1,2}$ More recently, the routine use of corticosteroid treatment as the 'gold standard of care' has resulted in prolonged ambulation and preservation of cardiac and respiratory function. ${ }^{3}$ The future for newly diagnosed boys with DMD looks even more promising since a new generation of drugs targeted towards RNA processing have been developed and include drugs designed to either readthrough nonsense mutations or skip exons with deletions or duplications (antisense oligonucleotides). ${ }^{4-6}$ Some of these agents are currently in phase 2 and phase 3 clinical trials, the first of these (translarna otherwise known as ataluren) has recently been given provisional regulatory approval in Europe. $^{7}$

1. Villanova M; Brancalion B; Mehta AD. Duchenne muscular dystrophy: life prolongation by noninvasive ventilatory support. Am J Phys Med Rehabil 2014;93:595-9.

2. Viollet L; Thrush PT; Flanigan KM; et al. Effects of angiotensin-converting enzyme inhibitors and/or beta blockers on the cardiomyopathy in Duchenne muscular dystrophy. Am J Cardiol 2012:110:98-102.

3. Griggs RC1, Herr BE, Reha A, et al. Corticosteroids in Duchenne muscular dystrophy: major variations in practice. Muscle Nerve 2013:48:27-31.

4. Touznik A; Lee JJ; Yokota T. New developments in exon skipping and splice modulation therapies for neuromuscular diseases. Expert Opin Biol Ther 2014 ;14:809-19.

5. Voit T; Topaloglu H; Straub V; et al. Safety and efficacy of drisapersen for the treatment of Duchenne muscular dystrophy (DEMAND II): an exploratory, randomised, placebo-controlled phase 2 study. Lancet Neurol 2014:13:987-96.

6. Veltrop M; Aartsma-Rus A. Antisense-mediated exon skipping: taking advantage of a trick from Mother Nature to treat rare genetic diseases. Exp Cell Res 2014;325:505.

7. Bushby K, Finkel R, Wong B, et al. PTC124-GD-007DMD STUDY GROUP. Ataluren treatment of patients with nonsense mutation dystrophinopathy. Muscle Nerve 2014;50:477-87. 


\title{
CIR-Myo News: Abstracts of the 2015 Spring Padua Muscle Days
}

\author{
Terme Euganee Padua (Italy), March 12 - 14, 2015
}

\section{Abstracts}

\section{The treatment of skeletal muscle channelopathies}

\section{Emma Matthews}

MRC Centre for Neuromuscular Diseases, ION, UCL and National Hospital for Neurology and Neurosurgery, Queen Square, London, UK

E-mail: emma.matthews@ucl.ac.uk

Skeletal muscle channelopathies are rare inherited disorders that are characterised by disabling episodes of muscle paralysis or myotonia. The periodic paralyses include hypokalaemic periodic paralysis, hyperkalaemic periodic paralysis, Andersen-Tawil syndrome and thyrotoxic periodic paralysis. The non-dystrophic myotonias include paramyotonia congenita, sodium channel myotonia and myotonia congenita. Accurate diagnosis forms the foundation of appropriate treatment. Symptoms are frequently triggered by specific provoking factors including certain foods, temperature or activity. Treatment can begin with education of these factors and how to avoid them. Exercise is often limited but careful technique and modification can allow many forms of activity to continue. Pharmacological therapies for the periodic paralyses include acetazolamide and diuretics (either potassium sparing or potassium losing). Emergency treatment of an attack of hypokalaemic periodic paralysis with intravenous potassium can sometimes be needed if there are ECG changes or severe muscle weakness causing respiratory compromise. Caution is needed however with very strict ECG and serum potassium level monitoring as a rebound hyperkalaemia can occur. Thyrotoxic periodic paralysis is most common in Asian populations. It may present with an attack of hypokalaemic paralysis without obvious systemic features of thyrotoxicosis but is an important differential to consider as treating the thyroid dysfunction abolishes attacks of periodic paralysis.

Andersen-Tawil syndrome is the only skeletal muscle channelopathy to also affect the heart and cardiology input is needed for any conduction abnormalities. Usually there is a triad of periodic paralysis, dysmorphic features and cardiac disease but the dysmorphic features can be very subtle and many patients are asymptomatic from a cardiac perspective prompting a high degree of suspicion of this diagnosis. Sodium channel blockers form the mainstay of treatment for the myotonic disorders. Pain is probably an underrecognised symptom and as a result under-treated. Simple analgesics are usually ineffective and although sodium channel blockers may provide some relief, this is often incomplete. A severe neonatal phenotype with respiratory compromise often requiring intensive care support has recently been recognised. Symptoms do seem to respond to sodium channel blockers in the majority but less than 20 children with this phenotype are so far described and two fatalities have been reported suggesting optimal management is yet to be determined. Although the most prominent symptoms in the channelopathies occur in an episodic manner a fixed proximal weakness can also occur. Its relationship to the frequency or severity of these episodic symptoms is unclear. This poses difficulties in knowing if treating the episodic attacks will prevent the development of myopathy, the onset of which seems to be at least in part unrelated to age with severe weakness sometimes described in children. An MRI can be helpful in distinguishing between the presence of oedema which suggests ongoing attacks that are amenable to treatment and fatty infiltration suggesting an irreversible myopathy. If irreversible myopathy is established consideration needs to be given to functional and home modifications.

1. Matthews E, Hanna MG. Repurposing of sodium channel antagonists as potential new anti-myotonic drugs. Exp Neurol. 2014 Nov;261:812-5. doi: 10.1016/j.expneurol. 2014.09.003. Epub 2014 Sep 10.

$$
* * * * *
$$

\section{Small molecules for novel therapeutic approaches in sarcoglycanopathy}

Elisa Bianchini (1), Roberta Sacchetto (2), Romeo Betto (3) and Dorianna Sandonà (1)

(1) CIR-Myo, Department of Biomedical Sciences, University of Padova; (2) Department of Animal Medicine Production and Health, University of Padova; (3) C.N.R. Institute of Neuroscience, Department of Biomedical Sciences, University of Padova, Italy

E-mail: dorianna.sandona@unipd.it

Sarcoglycanopathy is the name of rare autosomal recessive disorders affecting mainly the proximal musculature, hence belonging to type 2 Limb Girdle Muscular Dystrophy family (LGMD2). Age of onset and disease severity is variable, ranging from severe forms (with onset in the first decade and rapid evolution) to milder forms (with late onset and slow progression) ${ }^{1}$ Four different forms (LGMD2C, LGMD2D, LGMD2E, LGMD2F) are caused by defects in the genes coding for $\gamma$-, $\alpha$-, $\beta$ - and $\delta$-sarcoglycans (SG), respectively. It is important to note that about $75 \%$ of $\alpha-\mathrm{SG}, 59 \%$ of $\beta-\mathrm{SG}$, $40 \%$ of $\gamma$-SG and $57 \%$ of $\delta$-SG genetic defects are missense mutations. ${ }^{1}$ Until now, 52, 29, 20 and 8 different missense mutations have been reported for $\alpha-, \beta-, \gamma-$ and $\delta$-SG, respectively. Sarcoglycans form a tetramer, the SG-complex, member of the dystrophin associated protein complex and key element to assure membrane stability during muscle contraction. The presence of a missense mutation generates a folding-defective, although potentially functional sarcoglycan, that is rapidly recognized by the cell's quality control and targeted to proteasomal degradation. The loss or strong reduction of the mutated sarcoglycan leads to a secondary variable deficiency of the other sarcoglycans, thus compromising sarcolemma stability. Disease severity is strictly related to the residual level of sarcoglycans in the sarcolemma, with the most severe forms characterized by the almost complete loss of the proteins. Interestingly, the entire SG-complex can be rescued at the cell membrane by blocking different steps of the degradative pathway of the sarcoglycan mutants, ${ }^{2,3}$ a result that opens a new perspective for the therapy of these neglected diseases. In particular, we have designed two therapeutic approaches named "protein rescue" and "protein repair" strategy. The former aims "to save" the mutant from degradation, whereas the second intends "to assist" folding-defective sarcoglycans to reach the native or a native-like conformation and thus overcome the quality control check point. In both cases the recovered protein is expected to assemble with the SG partners and traffic toward the plasma membrane. The pharmacological 


\section{CIR-Myo News: Abstracts of the 2015 Spring Padua Muscle Days}

Terme Euganee Padua (Italy), March 12 - 14, 2015

\section{Abstracts}

inhibition of the E3 ligase HRD1, key element of the sarcoglycan degradative route, allowed the quantitative and functional rescue of an $\alpha$-SG mutant both in a cell model and in primary myotubes derived from a patient suffering from LGMD2D, ${ }^{4}$ validating the "protein rescue" strategy. For the "protein repair" strategy, we are testing a panel of 12 small molecules known as protein folding correctors screened for the treatment of cystic fibrosis. Although the way of action on sarcoglycans of these compounds is still unknown, we demonstrate in a heterologous cell model and in myotubes derived from an LGMD2D patient the accumulation of different $\alpha$-SG mutants, that are competent to assemble with the wild type partners and traffic to the cell membrane. ${ }^{5}$ These data represent the proof of principle of the two conceived pharmacological strategies which validation in vivo is currently undergoing. We believe our work is moving toward the development of a cure for the most frequently reported cases of sarcoglycanopathyr

1. Sandonà D, Betto R. Sarcoglycanopathies: molecular pathogenesis and therapeutic prospects. Expert Rev Mol Med 2009;11:e28. doi: 10.1017/S1462399409001203.

2. Gastaldello S, D'Angelo S, Franzoso S, et al. Inhibition of proteasome activity promotes the correct localization of disease-causing alpha-sarcoglycan mutants in HEK293 cells constitutively expressing beta-, gamma-, and delta-sarcoglycan. Am J Path 2008;173:170-181. doi: 10.2353/ajpath.2008.071146. Epub 2008 Jun 5.

3. Soheili T, Gicquel E, Poupiot $J$, et al. Rescue of sarcoglycan mutations by inhibition of endoplasmic reticulum quality control is associated with minimal structural modifications. Hum Mutat 2012;33:429-39. doi: 10.1002/humu.21659. Epub 2011 Dec 22

4. Bianchini E, Fanin M, Mamchaoui K, et al. Unveiling the degradative route of the V247M $\alpha$-sarcoglycan mutant responsible for LGMD-2D Hum Mol Gen 2014;23:374658. doi: 10.1093/hmg/ddu088. Epub 2014 Feb 23.

5. Sandonà $\mathrm{D}$, Sacchetto $\mathrm{R}$, Bianchini $\mathrm{E}$, et al. PCT/EP2013/075158, patent pending.

$$
* * * * *
$$

\section{Toward a mitochondrial therapy of muscular} dystrophies: a progress report

Alessandra Zulian (1,2), Marco Schiavone (1,3), Justina Šileikyte (1,2), Erika Rizzo (1,2), Elena Palma (1), Francesca Tagliavini (4), Bert Blaauw (1), Sudeshna Roy (4), Frank J. Schoenen (4), Mike Forte (5), Luciano Merlini (6), Nadir Mario Maraldi (6), Patrizia Sabatelli (6,7), Paola Braghetta (8), Francesco Argenton (3), Paolo Bonaldo (8), Paolo Bernardi $(1,2)$

(1) Department of Biomedical Sciences, University of Padova; (2) Consiglio Nazionale delle Ricerche Neuroscience Institute, Padova; (3) Department of Biology, University of Padova; (4) University of Kansas Specialized Chemistry Center, Lawrence, Kansas; (5) Vollum Institute, Oregon Health and Science University, Portland Oregon; (6) Laboratory of Musculoskeletal Cell Biology, Istituto Ortopedico Rizzoli, Bologna; (7)Consiglio Nazionale delle Ricerche, Institute of Molecular Genetics, Bologna; (8) Department of Molecular Medicine, University of Padova, Italy

E-mail: paolo.bernardi@unipd.it
Ullrich Congenital Muscular Dystrophy and Bethlem Myopathy are inherited muscle diseases due to mutations in the genes encoding the extracellular matrix protein collagen VI. Opening of the cyclosporin A-sensitive mitochondrial permeability transition pore (PTP) is a causative event in disease pathogenesis, and a potential target for therapy [1]. Over the years, we have tested disease models as well as patients for their response to cyclophilin inhibitors [1-4]; in parallel, we have developed novel PTP inhibitors through high-throughput screening methods. I will report on our progress in the development and treatment of muscular dystrophies with PTP inhibitors.

1. Irwin WA, Bergamin N, Sabatelli P, et al. Mitochondrial dysfunction and apoptosis in myopathic mice with collagen VI deficiency. Nat Genet 2003;35,267-71.

2. Merlini L, Angelin A, Tiepolo T, et al Cyclosporin A corrects mitochondrial dysfunction and muscle apoptosis in patients with collagen VI myopathies. Proc Natl Acad Sci U S A 2008;105:5225-29.

3. Tiepolo T, Angelin A, Palma E, et al. The cyclophilin inhibitor Debio 025 normalizes mitochondrial function, muscle apoptosis and ultrastructural defects in Col6a1-/myopathic mice. Br J Pharmacol 2009;157,1045-52.

4. Zulian A, Rizzo E, Schiavone M, et al. NIM811, a cyclophilin inhibitor without immunosuppressive activity, is beneficial in collagen VI congenital muscular dystrophy models. Hum Mol Genet 2014;23:5353-63.

$* * * * *$

\section{Functional data and response to exercise in McArdle's disease: from reporting to training patients' empowerment}

Andrea Martinuzzi, Carla De Conti, Marinela Vavla, Enrico Trevisi

IRCCS E. Medea, Conegliano Research Centre, Italy E-mail: andrea.martinuzzi@ lanostrafamiglia.it

McArdle's disease, the most common muscle glycogenosis, is characterized by exercise intolerance and recurrent exercise induced myoglobinuria. The condition is associated with severe limitation not only in the capacity to sustain even brief acute strenuous efforts, but also in the significant reduction of skeletal muscle aerobic metabolism. The exercise limitation is typically eased after 6-7 minutes of warm-up, giving rise to the so called "second wind" phenomenon, likely linked to the mobilization and muscle uptake of blood borne glucose as fuel to sustain muscle contraction. $20-30 \%$ of patients develop fixed weakness and muscle atrophy, especially in the upper limbs and report difficulties even for routine mild activities like walking. ${ }^{1}$. No causal treatment is available for this myopathy. The supplement of oral sugar prior to acute effort and the aerobic training have been showed to provide relief and improve functioning. Diet manipulation with preference to carbohydrates has been also proposed as management strategy. ${ }^{2}$ Prescriptions in this direction are regular part of the medical management of the disease and form the core of the indications given to patients.In spite of the available evidences however, when interviewed at follow-up visit, patients often admit not being able to consistently follow the 


\title{
CIR-Myo News: Abstracts of the 2015 Spring Padua Muscle Days
}

\author{
Terme Euganee Padua (Italy), March 12 - 14, 2015
}

\section{Abstracts}

instructions in terms of regular exercise, being either overwhelmed by the fear of suffering exercise induced pain and acute muscle damage, or just not being able to regulate the intensity and frequency of the exercise sessions. Given these premises we designed a one week patient-tailored program of intensive functional evaluations, including direct assessment of peak $\mathrm{VO} 2$ and customized oversighted training, followed by detailed indications for home-work. The session was followed by active weekly telephone monitoring. We tested our novel strategy on a first group of 8 molecularly defined McArdle's patients. The intensive session was well tolerated by all but one patient who complained of growing pain and was later found to be suffering for an herniated lumbar disc. No rise in CK was observed after $45 \mathrm{~min}$ exercise sessions repeated twice a day for 4 days. The functional evaluations confirmed the significant reduction in aerobic power but also the inappropriate response to even mild exercise (e.g. $12 \mathrm{~min}$ walking test). The first post training evaluation at 3 months was available for 3 subjects, and provides evidence for mild but consistent improvement in most functional measures. Most importantly, the program was successful in modifying patient behavior and improve attitude towards regular exercising and more appropriate dietary habits. An appropriately designed and customized program of training coupled with active monitoring appears as a promising and efficient strategy to address functional limitations and reduce disability in McArdle's patients.

1. Martinuzzi A, Sartori E, Fanin M, Nascimbeni A, Valente L, Angelini C, et al.Phenotype modulators in myophosphorylase deficiency. Annals of Neurology 2003;53(4):497-502.

2. Quinlivan R, Martinuzzi A, Schoser B. Pharmacological and nutritional treatment for McArdle disease (Glycogen Storage Disease type V) (Review) Cochrane database syst rev. 2014 Nov 12;11:CD003458. doi: 10.1002/14651858.CD003458.pub5.

$$
* * * * *
$$

\section{FES in genetic neuromuscular disorders}

Andrea Marcante (1,2), Alfonc Baba (1), Paola Cudia (1), Antonio Merico (1), Corrado Angelini (1), Stefano Masiero (2), Francesco Piccione (1)

(1) IRCCS Fondazione Ospedale San Camillo, Lido di Venezia; (2) UOC Medicina Fisica e Riabilitativa, Department of Neuroscience, University of Padova, Italy. E-mail: francesco.piccione@ospedalesancamillo.net

Functional electrical stimulation (FES) is a widely accepted rehabilitative treatment. Its effects have been extensively investigated in patients with stroke ${ }^{1}$ or spinal cord injury ${ }^{2}$ and FES is also currently used in clinical and rehabilitative management of patients presenting these diseases. Nevertheless, only few studies ${ }^{3,4}$ show that this kind of electrical stimulation can be useful also in neuromuscular diseases. Through the present report we will present a case series that shows our initial experience and the obtained results by the treatment with FES of patients affected by two different neuromuscular diseases: Myotonic Dystrophy (Type 1) and Hereditary Spastic Paraparesis. The aim of the study was to assess the feasibility and the usefulness of FES treatment in neuromuscular diseases, and also verify the presence of any adverse effects. We selected 4 patients affected by Myotonic Dystrophy and 4 patients affected by Spastic paraparesis and all subjects were treated as inpatients in IRCCS San Camillo Hospital, for 4 consecutive weeks. The FES devices used in IRCCS San Camillo Hospital are: Cycling-FES for lower limbs (Hasomed RehaStim2 MOTOmed ${ }^{\circledR}$ viva2) and peroneal neuroprosthesis FES for foot drop (NESS ${ }^{\circledR}$ L300TM). Functional tests and clinical scales [i.e. Medical Research Council (MRC) Scale for Muscle Strength; Active Range of Motion (ankle; knee); Timed Up \& Go; 6 Minutes Walking Test] were performed, before and after treatment, in order to assess the effects of therapy. Our preliminary results show that there are no adverse effects of FES treatment in patients affected by this kind of neuromuscular disorders. FES treatment also seems to be useful in improving functional performances during deambulation, but further research with a larger number of treated patients is required to confirm this data.

1. Howlett O, Lannin NA, Ada L, McKinstry C. Functional electrical stimulation improves activity after stroke: A systematic review with meta-analysis. Arch Phys Med Rehabil. 2015, in press.

2. Hamid S, Hayek R. Role of electrical stimulation for rehabilitation and regeneration after spinal cord injury: an overview. Eur Spine J 2008;17:1256-69.

3. Chisari C, Bertolucci F, Dalise S, Rossi B. Chronic muscle stimulation improves muscle function and reverts the abnormal surface EMG pattern in myotonic dystrophy: a pilot study. J Neuroeng Rehabil 2013;10:94.

4. Marsden J, Stevenson V, McFadden C, et al. The effects of functional electrical stimulation on walking in hereditary and spontaneous spastic paraparesis. Neuromodulation 2013;16:256-60.

$$
* * * * *
$$

\section{P1. Abnormal lactate levels affect motor performance in Myotonic Dystrophy type 1}

Caterina Tramonti, Stefania Dalise, Federica Bertolucci, Bruno Rossi, Carmelo Chisari

Unit of Neurorehabilitation, Department of Neuroscience, University Hospital, Pisa, Italy.

E-mail: caterina.tramonti@gmail.com

Myotonic Dystrophy type 1 is a dominantly inherited disease comprehending multiple features such as myotonia, muscle weakness and multisystemic involvement. Fatigue and exhaustion during exercise are two common symptoms presented by dystrophic patients, thus representing the most significant factors that could negatively influence their quality of life and compliance to rehabilitation programs. ${ }^{1}$ Mitochondrial abnormalities and a significant increase in oxidative markers had been previously reported, suggesting the hypothesis of a mitochondrial functional impairment in the pathogenesis of the disease. ${ }^{2}$ The study aims at evaluating oxidative metabolism efficiency in Myotonic Dystrophy patients, through analysis of lactate levels at rest and after an incremental exercise test, in order to propose a safe and valid method to guide specific aerobic training in rehabilitation 


\title{
CIR-Myo News: Abstracts of the 2015 Spring Padua Muscle Days
}

\author{
Terme Euganee Padua (Italy), March 12 - 14, 2015
}

\section{Abstracts}

program of these subjects. We analyzed, in 18 Myotonic Dystrophy patients, resting and exercise-related blood lactate values, as an indirect marker of oxidative metabolism, comparing results to 15 healthy subjects forming the control group. The exercise protocol consisted of a submaximal incremental exercise on an electronically calibrated treadmill: patients performed 11 consecutive steps consisting of walking for two minutes at a constant speed of $3 \mathrm{Km} / \mathrm{h}$, while the inclination degree ranged from $0^{\circ}$ at baseline to $2.5 \%$ for each step. This way the physical exercise was maintained in predominantly aerobic condition. Lactate levels were assessed through venous blood samples, collected at rest and at 5, 10 and 30 minutes after the end of the exercise. Analysis of the differences in mean values in the two groups were performed using the $\mathrm{Z}$ test. ${ }^{3,4}$ The results showed early exercise-related fatigue in Myotonic Dystrophy patients, as they performed a mean number of 9 steps, while controls completed the whole exercise. Moreover, while resting values of lactate were comparable between the patients and the control group $(\mathrm{p}=0,69)$, after the exercise protocol, dystrophic subjects reached higher values of lactate, at any recovery time $(p<0,05)$. These observations suggest an early activation of anaerobic metabolism, not proportional to workload executed, thus evidencing an alteration in oxidative metabolism of such dystrophic patients. This safe and validate method could be used to evaluate muscle oxidative metabolism and provide an efficient help on physical therapy to be prescribed in such patients.

1. Tramonti C, Dalise S, Bertolucci F, et al. Abnormal lactate levels affect motor performance in Myotonic Dystrophy type. Eur J Trans Myol - Basic Appl Myol 2014;24:255-60

2. Usuki F, Ishiura S: Expanded CTG repeats in myotonin protein kinase increase susceptibility to oxidative stress. Neuroreport 1998, 9: 2291-2296.

3. Dalise S, Bertolucci F, Simonella C, Rossi B, Chisari C: Intensive aerobic training improves motor performances and oxidative metabolism efficiency in chronic polymyositis: A case report Neuromuscular Disorders 2012, 22: 221-225.

4. Bertolucci F, Neri R, Dalise S, Venturi M, Rossi B, Chisari C Abnormal Lactate levels in patients with Polymyositis and Dermatomyositis: the benefits of a specific Rehabilitative program, European Journal of Phisical and Rehabilitation Medicine, in press.

$$
* * * * *
$$

\section{The ERG1a K+ Channel Increases Intracellular Calcium Concentration and Calpain Activity in Skeletal Muscle Cells}

Amber Pond (1,2), Kevin Bradley (1), Evan Pratt (2), Ian Soderling (2), Wen-Horng Wang (2), and Gregory

$$
\text { Hockerman (2) }
$$

(1) Anatomy Department, Southern Illinois School of Medicine, Carbondale, IL, U.S., (2) Medicinal Chemistry and Molecular Pharmacology, Purdue University, West Lafayette, IN, U.S.

E-mail: apond@siumed.edu
In heart, the functional ERG1 (ether-a-gogo related gene) $\mathrm{K}^{+}$ channel is composed of both ERG1a and $1 \mathrm{~b}$ splice variant proteins and is known to be partially responsible for late phase repolarization of the cardiac action potential. $\left.{ }^{1,2}\right] \mathrm{We}$ have shown that the mouse ERG1a (MERG1a) splice variant protein is increased in skeletal muscle of mice experiencing atrophy as a result of hindlimb suspension (disuse). Additionally, we have shown that ectopic expression of Merg1a in mouse skeletal muscle (a result of the electroporation of expression plasmid into gastrocnemius muscle) increases activity of the ubiquitin proteasome pathway (UPP) ${ }^{3}$ ] a proteolytic pathway responsible for the majority of protein degradation that contributes to muscle loss in atrophic skeletal muscle. ${ }^{4}$ Further, we have shown that this ectopic expression increases protein levels of the UPP component E3 ligase MURF1, although not that of ATROGIN1. Because the calcium activated enzyme calpain is also known to contribute to proteolysis in skeletal muscle atrophy, we wanted to determine if Merg1a expression would also affect intracellular calcium levels and calpain activity. Thus, we infected $\mathrm{C} 2 \mathrm{C} 12$ myotubes with an adenovirus containing the human ERG1a (HERG1a) construct and determined that, as in mouse muscle, ERG1a expression increased levels of MURF1 protein, but not that of Atrogen. Interestingly, it also increased levels of intracellular calcium in $\mathrm{C} 2 \mathrm{C} 12$ myotubes by $86 \%$ in response to depolarization. Further, Merg1a expression also increased calpain activity in mouse gastrocnemius muscle by $58.8 \%$ ( $<<0.1$, relative to day 0 ) at day 3 after electroporation and by $79.4 \%$ ( $<<0.1$, relative to day 0) 4 days after electroporation.

1. Curran ME, Splawski I, Timothy KW, Vincent GM, Green ED, Keating MT. Cell 1995; 80:795-803.

2. Jones E. M., Roti Roti E. C., Wang J., Delfosse S. A., Robertson G. A. J. Biol. Chem.2004; 279:44690-94.

3. Wang X, Hockerman GH, Green 3rd HW, Babbs CF, Mohammad SI, Gerrard D, Latour, MA, London B, Hannon KM, Pond AL. FASEB J 2006; 20(9):1531-3.

4. Franch HA, Price SR. Curr Opin Clin Nutr Metab Care 2005; 8:271-275.

\section{P8. Age upper limits for everyday key tasks: indications from the decline of the Master World Record Series}

$$
\text { Paolo Gava (1), Helmut Kern (2,3), Ugo Carraro (4) }
$$

(1) CIR-Myo, Department of Biomedical Science, University of Padova, Italy; (2) Ludwig Boltzmann Institute of Electrical Stimulation and Physical Rehabilitation, Vienna, Austria; (3) Department of Physical Medicine, Wilhelminenspital, Vienna, Austria; (4) IRRCS Fondazione Ospedale San Camillo, Venezia, Italy

E-mail: paologavastra@alice.it

The power developed by the skeletal muscle decreases with aging: no question. The decline rate can be deduced from the decline of the world records of Masters athletes in various track and field discipliness. ${ }^{1,2}$ Studies on this subject are numerous and the results of our, in line with those of others, indicate trend-lines for the power decline starting at the age of 30 and pointing to zero at the age of 110 years. ${ }^{3,4}$ The normalized decline rate (from 1 to 0 ) of the skeletal muscle power with aging of any ordinary people, if always healthy, 


\title{
Eur J Transl Myol/Basic Appl Myol 2015; 25 (3): 145-182 \\ CIR-Myo News: Abstracts of the 2015 Spring Padua Muscle Days
}

\author{
Terme Euganee Padua (Italy), March 12 - 14, 2015
}

\section{Abstracts}

is the same of the normalized decline rate of the world records of the Masters athletes. The most important everyday functional tasks can be related to the main disciplines of the track and field competitions. A short walk can be associated to the short runs; a long walk to the endurance runs; the stairs climb $^{1}$ to the jumping events (all imply mainly the legs power to raise the body centre of gravity) and finally the raise of a weighted supermarket bag on to the kitchen table can be associated to the throwing events (they require mainly the power of the upper limbs). ${ }^{4,5}$ The decline rate of the Masters world records for selected events (short runs, long runs, simple jumps and selected throwing events) have been analysed and plotted vs age. The declining trend lines of the Masters word records can indicate the upper level of the age limits of the key everyday tasks for everyone (short walk; long walk, stairs climb and bag raise). Accepting some simple assumptions for the performance of the main functional tasks (limiting power from 20\%, to 5\% of the youthful power), then the limit age ranges from 85 to over 105 years. An earlier occurrence is, thus, the product of a disease, severe muscle disuse included.

U.C. thanks the Interdepartmental Research Center of Myology at the Department of Biomedical Sciences, University of Padova, Italy for collaboration and hospitality and the Ludwig Boltzmann Institute of Electrical Stimulation and Physical Rehabilitation of Vienna at the Department of Physical Medicine, Wilhelminenspital, Vienna, Austria for support and collaboration.

1. Hill AV. The physiological basis of athletic records. Sci Monthly 1925;21: 409 - 28.

2. Mitchell W K, Williams J, Atherton P. Sarcopenia, dynapenia, and the impact of advancing age on human skeletal muscle size and strength; a quantitative review. Frontiers in Physiology; 2012; 3: 1-18.

3. Gava P, Kern H, Carraro U. Age-associated power decline from running, jumping, and throwing male Masters world records. Experimental Aging Research 2015;41, in press.

4. Baker AB, Tang, YQ, Turner MJ. Percentage decline in masters superathlete track and field performance with aging. Exp Aging Res 2003;29, 47-65.

5. Runge M, Rittweger J, Russo CR, Schiessl H, Felsenberg D. Is muscle power output a key factor in the age-related decline in physical performance? A comparison of muscle cross section chair-rising test and jumping power. Clin Physiol Funct Imaging 2004;24:335-40.

$$
* * * * *
$$

\section{Role of tissue niche in aging and neuromuscular disease}

Laura Pelosi, Laura Forcina, Elisa Vizzaccaro, Antonio $\underline{\text { Musarò }}$

(1) Institute Pasteur Cenci Bolognetti, Unit of Histology and Medical Embryology, IIM, Sapienza University of Rome, Italy

E-mail: "Antonio Musaro" >antonio.musaro@ uniroma1.it>

The prolongation of skeletal muscle strength in neuromuscular disease has been the objective of numerous studies employing a variety of approaches. Stem cell therapy represents a promising tool to cure genetic diseases.
However, this approach is not definitive yet and several hurdles limit the immediate translation of this strategy into clinic. One of the crucial parameters of tissue regeneration is the microenvironment in which the stem cell populations should operate. ${ }^{1}$ Stem cell microenvironment, or niche, provides essential cues that regulates stem cell proliferation and that directs cell fate decisions and survival. It is therefore plausible that loss of control over these cell fate decisions might lead to a pathological transdifferentiation and contribute to the exacerbation of a pathologic condition, such as muscular dystrophy. Among critical parameters, the activation and persistence of inflammatory and fibrotic pathways may render the dystrophic muscle incapable to sustain and complete an efficient muscle regeneration, ${ }^{2}$ leading to a progressive loss of muscle tissue due to chronic degeneration of muscle and to the exhaustion of satellite cells that replace damaged fibers. ${ }^{3}$ Indeed, the progressive loss of tissue function and integrity observed in dystrophic muscles are the eventual consequences of a history of continuous rounds of degeneration and regeneration. Specific factors are required to trigger stem cells toward a specific lineage, to improve their survival, and to render them effective in contributing to tissue repair. Studies on stem cell niche leaded to the identification of critical players and physiological conditions that improve tissue regeneration and repair. Preliminary evidences demonstrated that the local form of Insulin-like Growth Factor-1 (mIGF-1) ${ }^{4,5}$ sustains muscle hypertrophy and regeneration in senescent skeletal muscle, enhances the recruitment of circulating stem cells in injured muscle and counteracts muscle wasting in $\mathrm{mdx}$ dystrophic mice, reducing the inflammatory response and improving muscle mass and strength and elevating pathways associated with muscle survival and regeneration. Among the factors modulated by mIGF-1, we observed a specific downregulation of the inflammatory cytokines IL-6, which has been associated with the switch from acute to a chronic inflammatory response that therefore can exacerbate the dystrophic phenotype. We will discuss the role of mIGF-1 and IL-6 in the modulation of muscle regeneration under physiological and pathologic conditions.

1. Carosio S, Berardinelli MG, Aucello M, Musaro A. 2011. Impact of ageing on muscle cell regeneration. Ageing Res Rev 10, 35-42.

2. Porter JD, Khanna S, Kaminski HJ, Rao JS, Merriam AP, Richmonds CR, Leahy P, Li J, Guo W, Andrade FH. 2002. A chronic inflammatory response dominates the skeletal muscle molecular signature in dystrophindeficient mdx mice. Hum Mol Genet 11:263 - 272.

3. Cohn RD, Campbell KP. 2000. Molecular basis of muscular dystrophies. Muscle Nerve 23:1456 - 1471.

4. Musaro A, McCullagh K, Paul A, Houghton L, Dobrowolny G, Molinaro M, Barton ER, Sweeney HL, Rosenthal N. 2001. Localized Igf-1 transgene expression sustains hypertrophy and regeneration in senescent skeletal muscle. Nat Genet 27:195 - 200.

5. Scicchitano BM, Rizzuto E, and Musarò A. 2009 Counteracting muscle wasting in aging and neuromuscular diseases: the critical role of IGF-1. Aging; 1: $451-457$ 


\title{
CIR-Myo News: Abstracts of the 2015 Spring Padua Muscle Days
}

\author{
Terme Euganee Padua (Italy), March 12 - 14, 2015
}

\section{Abstracts}

\section{Exercise in sedentary elderlies: effects on muscle trophism and MCU expression}

Sandra Zampieri (1,2), Simone Mosole (1,2), Stefan Löfler (2), Hannah Fruhmann (2), Samantha Burggraf (2), Antonio Musarò (3), Marco Sandri (1,4), Ján Cvečka (5), Dušan

Hamar (5), Milan Sedliak (5), Veronica Tirptakova (5), Nejc

Šarabon (6), Cristina Mammuccari (1), Ugo Carraro (7), Helmut Kern (2)

(1) Department of Biomedical Sciences, Padova, Italy; (2) Ludwig Boltzmann Institute of Electrical Stimulation and Physical Rehabilitation, Vienna, Austria; (3) DAHFMO-Unit of Histology and Medical Embryology, IIM, Sapienza University of Rome, Italy. (4) Venetian Institute of Molecular Medicine, Dulbecco Telethon Institute; (5) Faculty of Physical Education and Sport, Comenius University, Bratislava, Slovakia; (6) University of Primorska, Science and Research Centre, Institute for Kinesilogical Research, Koper, Slovenia; (7) IRRCS Fondazione Ospedale San Camillo, Venezia, Italy.

E-mail: sanzamp@unipd.it

Ageing is associated with detrimental changes in function, mass and structure of skeletal muscle that are also predisposing factors to disability and increased the risk of falling in elderlies. Lifestyle interventions including increased physical activity are one of the primary approach to prevent age related muscle decline. Our recent results from a peculiar group of lifespan trained seniors, suggest that long term physical exercise have beneficial effects on age related decay not only affecting muscle trophism and phenotype, but also counteracting denervation atrophy by promoting reinnervation. ${ }^{1-3}$ By this rationale, we trained 70 years sedentary seniors either with leg press (LP) or electrical stimulation (ES) for 9 weeks. We investigated the effects of training on mobility and muscle trophism by functional tests and morphological, biochemical and molecular analyses of Vastus Lateralis muscle biopsies, before and after the training. The effects of increased muscle activity with respect to sedentary lifestyle were also analysed focusing on the effect on mitochondrial proteins associated with $\mathrm{Ca}^{2+}$ uptake and respiratory chain function. The results show that either LP or ES training induce similar force and functional improvements without damaging skeletal muscle fibers. Indeed, ES more efficiently in comparison to LP, attenuate muscle mass decline maintaining the overall size of muscle fibers and increasing the number and the size of the fast type fibers, also activating satellite cells. Both trainings, without significantly affecting the overall number of mitochondria and respiratory chain enzymes (SDH and COXIV), induced an increased expression of MCU regulating the mitochondria $\mathrm{Ca}^{2+}$ uptake in post training biopsies, which seems to be associated to the increase of fast fibers diameter in ES trained subjects. ${ }^{4-6}$ Altogether these findings show that training sedentary elderlies with ES is a safe and effective intervention to counteract muscle fiber atrophy and to improve the performances of aging muscles.

U.C. thanks the Interdepartmental Research Center of Myology at the Department of Biomedical Sciences, University of Padova, Italy for collaboration and hospitality and the Ludwig Boltzmann Institute of Electrical Stimulation and Physical Rehabilitation of Vienna at the Department of
Physical Medicine, Wilhelminenspital, Vienna, Austria for support and collaboration.

1. Kern H, Barberi L, Löfler S, et al. Electrical stimulation counteracts muscle decline in seniors. Front Aging Neurosci 2014;6:189. doi: 10.3389/fnagi.2014.00189. eCollection 2014.

2. Mosole S, Carraro U, Kern H, et al. Long-term high-level exercise promotes muscle reinnervation with age. J Neuropathol Exp Neurol 2014;73:284-94. doi: 10.1097/NEN.0000000000000032.

3. Zampieri S, Pietrangelo L, Loefler S et al. Lifelong physical exercise delays age-associated skeletal muscle decline. J Gerontol A Biol Sci Med Sci 2015;70:163-73. doi: 10.1093/gerona/glu006. Epub 2014 Feb 18.

4. Patron M, Raffaello A, Granatiero V, et al. The mitochondrial calcium uniporter (MCU): molecular identity and physiological roles. J Biol Chem 2013;288:10750-8. doi: 10.1074/jbc.R112.420752. Epub 2013 Feb 11.

5. Rizzuto R, De Stefani D, Raffaello A, Mammucari C. Mitochondria as sensors and regulators of calcium signalling. Nat Rev Mol Cell Biol 2012;13:566-78. doi: 10.1038/nrm3412. Epub 2012 Aug .

6. Mammucari C, Gherardi, G, Zamparo, et al. (2015) The Mitochondrial Calcium Uniporter controls skeletal muscle trophism in vivo. Cell reports, in press.

$* * * * *$

\section{Migrating tumor cells and muscle wasting: Role of DOG1 (Discovered On Gastrointestinal Stromal Tumor 1) in tumor cell motility and skeletal muscle colonization}

Emanuele Berardi (1), Daniela Annibali (2), Ilaria Perini (1), Marco Cassano (3), Carolina Ortiz (1), Simona Ultimo (1), Domiziana Costamagna (1,4), Paola Costelli (4), Antonio Di Grazia (5), Hanne Grosemans (1), Maurilio Sampaolesi (1)

(1) Translational Cardiomyology Laboratory, Department of Development and Reproduction, KUL University of Leuven Leuven, Belgium; Interuniversity Institute of Myology Italy; (2) Laboratory of Gynecological Oncology, Oncology Department, University Hospitals Leuven,KU Leuven, Belgium; (3) School of Life Sciences, Ecole Polytechnique Fédérale de Lausanne Lausanne, Switzerland; (4) Unit of Experimental and Clinical Pathology, Department of Clinical and Biological Sciences, University of Turin, Turin, Italy; (5) Istituto Pasteur Fondazione Cenci Bolognetti, Department of Biochemical Sciences "A. Rossi Fanelli", Sapienza University of Rome, Italy.

E-mail: Emanuele.Berardi@med.kuleuven.be

Cancer cachexia is a degenerating syndrome characterized by a severe body weight loss occurring in the advanced stage of tumor progression. Muscles wasting, together with a remarkable loss of fat tissue, are known hallmarks of cancer cachexia. Clinically, cancer-related cachexia accounts for almost 2 million of deaths per year and until $80 \%$ of tumors can develop cachexia. So far, many studies identified a pivotal role of several circulating tumor factors (i.e. proteolysis-inducing factor, PIF) in mediating muscle degeneration observed in skeletal muscle tissue. In particular, pro-inflammatory cytokines (i.e. IL-1, IL-6 and TNF- $\alpha$ ) from 


\title{
Eur J Transl Myol/Basic Appl Myol 2015; 25 (3): 145-182 \\ CIR-Myo News: Abstracts of the 2015 Spring Padua Muscle Days
}

\author{
Terme Euganee Padua (Italy), March 12 - 14, 2015
}

\section{Abstracts}

tumor origin are the main players in triggering muscle wasting. ${ }^{1}$ They sustain the inflammatory network between tumor and skeletal muscle tissue accountable for the main catabolic pathways activated into the skeletal muscle fibers such as the ubiquitin-proteasome pathway (UPP), proteasesmediated degradation and autophagocytosis. ${ }^{2}$ Since the inflammatory burden is considered the most important mediator of muscle degenerative processes, many efforts pointed to the treatment of cancer-related cachexia with antiinflammatory drugs. Nevertheless, soluble antibodies against TNF- $\alpha$ and their receptors still showed poor beneficial effects in the clinics.We investigated cancer-related cachexia in Gastrointestinal Stromal Tumor (GIST) xenograft-mouse model. GIST is the most common mesenchymal tumor of the gastrointestinal tract, characterized by high expression of KIT, PDGFR ${ }^{3}$ and DOG1, a calcium activated chloride channel. ${ }^{4}$ By transplanting of human GIST fragments in nude mice, we found that $30 \%$ of tumor bearing mice developed severe muscle wasting characterized by a strong reduction muscle fibers area associated with increase of Atrogin1 expression, a known marker of muscle atrophy. ${ }^{5}$ Expression profile analysis of inflammatory markers showed that muscle wasting in cachectic mice was not sustained by the increasing of pro-inflammatory cytokines from tumor, nor by changes in their expression in skeletal muscles. Furthermore, histological analysis revealed the presence of tumor cells within the connective tissue surrounding the fibers. In vivo analyses showed that both tumor cell migration and colonization of cachectic muscles are processes closely associated with the expression of DOG1. Loss of function experiments further supports our hypothesis. Finally, histological analysis performed on skeletal muscle tissues of colon carcinoma C26-bearing mice shows the colonization of tumor cells in muscle microenvironment. Thus, tumor cells can also colonize skeletal muscle tissues in immunocompetent murine model of cancer cachexia.

1. Tisdale MJ. Mechanisms of cancer cachexia. Physiol rev 2009:89, 381-410.

2. Fearon KC, Glass DJ, Guttridge DC. Cancer cachexia: mediators, signaling, and metabolic pathways. Cell metabolism 2012;16:153-66.

3. Reichardt P, Hogendoorn PC, Tamborini E, et al. Gastrointestinal stromal tumors I: pathology, pathobiology, primary therapy, and surgical issues. Seminars in oncology 2009;36:290-301.

4. Wada T, Tanabe S, Ishido K, et al. DOG1 is useful for diagnosis of KIT-negative gastrointestinal stromal tumor of stomach. World Journal of Gastroenterology : WJG 2013:19,9133-6.

5. Gomes MD, Lecker SH, Jagoe RT, et al. Atrogin-1, amuscle-specific F-box protein highly expressed during muscle atrophy. Proc Natl Acad Sci U S A. 2001;98:14440-5. Epub 2001 Nov 20.

\section{Perilipins in human muscle aging and atrophy}

Maria Conte (1), Francesco Vasuri (2), Enrico Bertaggia (3), Andrea Armani (3), Alessio Degiovanni (2), Antonia

D’Errico-Grigioni (2), Marco V Narici (5), Marco Sandri (3), Claudio Franceschi (1), Stefano Salvioli (1)

(1) Department of Experimental, Diagnostic and Specialty Medicine (DIMES) and Interdepartmental Centre "L. Galvani” (CIG), University of Bologna, 40126 Bologna, Italy: (2) "F. Addarii" Institute of Oncology and Transplant Pathology, Department of Experimental, Diagnostic and Specialty Medicine, S.Orsola-Malpighi Hospital, University of Bologna, 40138 Bologna, Italy; (3) Dulbecco Telethon Institute, Venetian Institute of Molecular Medicine, 35129 Padova, Italy; (4) Reconstructive Hip and Knee Joint Surgery, Istituto Ortopedico Rizzoli, Bologna Italy; (5) School of Graduate Entry Medicine and Health, Division of Clinical Physiology, Derby Royal Hospital, University of Nottingham, Derby, DE22 3DT, UK

E-mail:m.conte@unibo.it

Human aging is characterized by the progressive loss of muscle mass and strength, phenomenon known as sarcopenia. The etiology of sarcopenia is still not clear and involves several factors. Among these, sedentary lifestyle acts synergistically with age to determine the decrease of muscle mass and strength. ${ }^{1}$ Aging and inactivity play a role also in the accumulation of inter-muscular adipose tissue, another factor involved in the development of sarcopenia. ${ }^{2}$ In skeletal muscle, fat accumulates also as intra-muscular triglycerides (IMTGs). Little is known about the possible role of IMTGs in muscle aging and atrophy. IMTGs accumulate in form of lipid droplets (LDs) characterized by the presence of Perilipins (Plins). In skeletal muscle the most abundant are Plin2 and Plin5. The exact role of these Plins is currently unknown, however it is well established that these proteins correlate positively with the levels of IMTG and are upregulated by lipid accumulation under both physiological and pathological conditions. ${ }^{3}$ Recently, we found that the expression level of Plin2 increases with age and inactivity, and it is inversely associated with muscle mass and strength. ${ }^{4}$ On the basis of these findings, we compared the expression of Plin2 and Plin5 during muscle aging and inactivity, and analysed the possible consequences of such expression on muscle mass and strength. To this purpose, we investigated the levels of these two proteins in Vastus lateralis muscle biopsies from subjects of different age: healthy donors and patients with limb mobility impairment, that gave us the possibility to analyse the expression level of Plins in condition of chronic physical inactivity. Our data indicate that of Plin2, but not Plin5, increases with age and inactivity. Moreover Plin2, but not Plin5, is associated not only with active form of $\mathrm{p} 53$, but also with muscle atrophy-related genes, such as MuRF-1 and Atrogin, suggesting a role only for Plin2 in muscle aging and atrophy. To further reinforce our observation on this association, we took advantage of an experimental model of muscle atrophy induced by cutting the sciatic nerve. In this model, an increase in Plin2 and a concomitant decrease of Plin5 was observed, adding further evidence that these proteins have different role in skeletal muscle and notably during atrophy. 


\title{
Eur J Transl Myol/Basic Appl Myol 2015; 25 (3): 145-182 \\ CIR-Myo News: Abstracts of the 2015 Spring Padua Muscle Days
}

\author{
Terme Euganee Padua (Italy), March 12 - 14, 2015
}

\section{Abstracts}

1. Breen L, Phillips SM. Interactions between exercise and nutrition to prevent muscle waste during aging. Br J Clin Pharmacol 2013;75:708-15. doi: 10.1111/j.13652125.2012.04456.x.

2. Marcus RL, Addison O, LaStayo PC. () Intramuscular adipose tissue attenuates gains in muscle quality in older adults at high risk for falling. A brief report. J Nutr Health Aging. 2013;17:215-8. doi: 10.1007/s12603-0120377-5.

3. Bosma M, Sparks LM, Hooiveld GJ, et al. Overexpression of PLIN5 in skeletal muscle promotes oxidative gene expression and intramyocellular lipid content without compromising insulin sensitivity.

4. Conte M, F Vasuri, G Trisolino, et al. Increased Plin2 expression in human skeletal muscle is associated with sarcopenia and muscle weakness. PLoS One. 2013;8:e73709. DOI: 10.1371/journal.pone.0073709

$$
* * * * *
$$

\section{Ergo-Cell: A multi-sensor system offering new} opportunities for research in elderly

\section{Nejc Sarabon (1,2), Miha Perosa (1), Jure Praznikar (1), Helmut Kern (3)}

(1) University of Primorska, Andrej Marusic Institute, Department of Health Study, Koper, Slovenia; (2) S2P, Science to Practice, d.o.o., Laboratory for Motor Control and Motor Behaviour, Ljubljana, Slovenia; (3) Wilhelminenspital, Ludwig Boltzmann Institute of Electrical Stimulation and Physical Rehabilitation, Vienna, Austria. E-mail: nejc.sarabon@s2p.si

Interest, development and market offer of sensor based systems have grown tremendously over the last few years. Today technology allows us to monitor all segments of our lives, from health aspects to living environment and others. Current multi-sensor systems usually operate in a way that the measured data are stored and analysed only later (post hoc) by the user. Ergo-Cell is a novel multi-sensor device that offers the ability to transfer data from multiple sensors to the cloud in real time. Coach, doctor or any other end user can $\log$ into a web based application from anywhere in the World and check and/or compare the measured parameters from one or more remote systems simultaneously. Ergo-Cell is an embedded modular system composed of three main parts: i) the base board, ii) the battery module and iii) wireless sensors. The biggest challenge while developing the Ergo-Cell was the ability to use multiple sensors without affecting the measuring performance. A real time operating system is therefore used to ensure that all operations are executed on every cycle at a given time, without any delay. The beating heart of the new device is the ARM Cortex-M3 microcontroller; FreeRTOS is used for the operating system. ${ }^{1}$ The program runs on multiple threads, one task reads data of board sensors (temperature, humidity, accelerometer and gyroscope), the second reads data from the GPS (Global Positioning System), while the third task takes care for the wireless communication etc. ANT+ is the main wireless technology that allows devices to talk to each other. The main feature is the interoperability which means that ANT+ products can be used from multiple brands ${ }^{2}$ The first version of EC incorporates ANT module for reading heartbeat data, later on we plan to extend the list of supported sensors to cadence, speed, blood pressure and other. We also plan to take ANT+ advantages and produce custom wireless sensors for our needs. The last important part of the new multi-sensor system is the GPRS (General Packet Radio Service) modem, used for sending data to the cloud. EC is connected to the server in a "transparent" mode so bidirectional communication is possible in real time. The dimensions of the first Ergo-Cell version are $10 \times 10 \times 5 \mathrm{~cm}$, weight is about $500 \mathrm{~g}$, battery life $20+$ hours. We believe that Ergo-Cell offers new interesting opportunities for studies in the field of general mobility, ergonomics and specific physical activity in elderly.

1. http://www.freertos.org

2. http://www.thisisant.com/consumer/ant-101/what-is-ant

$* * * * *$

Effect of high versus normal intensity acute resistance exercise on bone metabolism in pre- and postmenopausal women: rationale and design

Jana Kovárová, Dušan Hamar, Peter Schickhofer, Ján

Cvečka, Veronika Tirpáková, Lubica Böhmerová, Matej Vajda, Milan Sedliak

Department of Sport Kinantropology, Faculty of Physical Education and Sports, Comenius University in Bratislava, Slovakia.

E-mail: "Jan_Cvecka" <cvecka@fsport.uniba.sk>

The study will examine acute response of bone turnover in pre- and postmenopausal women to resistance exercise. ${ }^{1}$ A randomized repeated measures crossover design will be used to find out the effect of two single sessions of resistance exercise on serum bone metabolism markers: bone alkaline phosphatase (bALP), amino-terminal propeptide of type 1 procollagen (P1NP), beta-crosslaps (bCTx) and sclerostin. All the subjects will perform one session with a constant resistance $(75 \%$ of $1 \mathrm{RM})$ and one isokinetic session with repeated $(10 \mathrm{~Hz})$ counter-movements in concentric and accelerations in eccentric phase of the movement on different days. Each session will consist of 6 sets of 6 repetitions with a 2-minute rest and will be conducted between 8 and 9 AM. Blood samples will be obtained by venipuncture of antecubital vein before (after a 10-12 hour over-nigh fast), 15 minutes, 24 and 48 hours after the exercise.

1. Zampieri S, Pietrangelo L, Loefler S, et al. Lifelong physical exercise delays age-associated skeletal muscle decline. J Gerontol A Biol Sci Med Sci. 2015 Feb;70(2):163-73. doi: 10.1093/gerona/glu006. Epub 2014 Feb 18

$$
* * * * *
$$

P2. Muscle analysis to assess motor impairment in cancer patients

Caterina Tramonti, Stefania Dalise, Federica Bertolucci, Bruno Rossi, Carmelo Chisari

Unit of Neurorehabilitation, Department of Neuroscience, University Hospital, Pisa, Italy

E-mail: c.chisari@ao-pisa.toscana.it 


\section{CIR-Myo News: Abstracts of the 2015 Spring Padua Muscle Days}

Terme Euganee Padua (Italy), March 12 - 14, 2015

\section{Abstracts}

Cachexia is a metabolic syndrome characterized by skeletal muscle wasting and weight loss, associated with an underlying disease and chronic inflammation. Cancer cachexia affects the majority of cancer patients, leading to weakness, decreased mobility and quality of life and negatively interfering with anticancer therapies. Furthermore, no effective therapy of cachexia exists as far. ${ }^{1,2}$ One of the reasons of this failure is the lack of a deep knowledge of the basic mechanisms underlying muscle wasting in this condition $^{3}$ The goal of this study is to characterize muscle deficits in cancer cachexia, and to develop novel approaches to counteract cachexia. The muscular function of 15 oncologic patients ( 13 males, 2 females, $47.07 \pm 17.58$ years of age) and 16 controls (13 males, 3 females, $49.13 \pm 17.55$ years of age) was analyzed in this study, by means of: (a) the analysis of the oxidative metabolism, indirectly evaluated trough dosage of blood lactate levels before and after a submaximal incremental exercise on a treadmill; (b) the analysis of strength and resistance, in both proximal and distal muscles, by means of an isokinetic dynamometer, expressed as the average of strength applied during 3 maximal voluntary contractions (MVC) lasting 3 seconds each and the decay of the strength exerted, expressed as a percentage of maximum voluntary contraction (\%MVC) during an isometric contraction sustained for 60 seconds. Results were then analyzed and compared with obtained by a group of healthy subjects. Mann-Whitney U test was used for statistical analysis. Patients were affected by solid tumors of different severity located in different sites (neck, lung, testicle and neuroendocrine tumors) and in different stages of disease. During the study patients were being subjected to chemotherapic treatments. Analysis of oxidative metabolism during the incremental exercise on the treadmill showed that patients performed a reduced number of steps respect to the controls. Lactate levels were significantly $(\mathrm{p}<0.05)$ higher in patients than in the control group at each blood sample. Muscle strength analysis showed a reduction of MVC during the isometric contraction and also a reduction of endurance, expressed as $\% \mathrm{MVC} / 60 \mathrm{~s}$ in the patients group. The data emerging from this study highlight a precocious fatigability and an impairment of muscle oxidative metabolism in subject affected by a pre-cachectic stage of cancer. A trend of precocious fatigability and an impairment of muscle strength production was also observed. These data underline the relevance of assessing muscle function in order to develop novel rehabilitative approaches able to counteract the motor impairment and eventually to prevent cachexia in these patients.

1. Evans WJ Morley JE, Argilés J, et al. Cachexia: a new definition Clin Nutr 2008; 27: 793-9. doi: 10.1016/j.clnu.2008.06.013. Epub 2008 Aug 21.

2. Evans WJ. Skeletal muscle loss: cachexia, sarcopenia, and inactivity.Am J Clin Nutr 2010;91:1123S. doi: 10.3945/ajcn.2010.28608A. Epub 2010 Feb 17.

3. Gomes MD, Lecker SH, Jagoe RT, Navon A, Goldberg AL. Atrogin-1, a muscle-specific F-box protein highly expressed during muscle atrophy Proc Natl Acad Sci U.S.A 2001;98: 14440-5. Epub 2001 Nov 20.

\author{
Novel routes for muscle mass regulation: focus on \\ mitochondria
}

Caterina Tezze (1)*, Vanina Romanello (1)*, Tatiana Varanita (1,2), Mariam Desbats (3), Maria Eugenia Soriano $(1,2)$, Alberto Casarin (3), Mattia Albiero (1), Stefan Loefler (4), Helmut Kern (4), Bert Blaauw (1), Sandra Zampieri (5), Leonardo Salviati (3), Luca Scorrano (1,2), Marco Sandri

(1) Venetian Institute of Molecular Medicine, Padova, Italy; (2) Department of Biology, University of Padova, Italy; (3) Clinical Genetics Unit, Department of Woman and Child Health, University of Padova, Italy; (4) Ludwig Boltzmann Institute of Electrical Stimulation and Physical Rehabilitation, Vienna, Austria; (5) Department of Biomedical Science, University of Padova, Italy.

E-mail: marco.sandri@unipd.it

*Equal contribution The maintenance of a functional mitochondrial network is particularly important in highly structured and metabolically active tissues like muscle, but whether and how changes in mitochondrial fusion and fission cause extramitochondrial responses at the cellular and organismic level is unknown. Here we show that age related muscle loss and weakness in humans (sarcopenia) correlates with the decreased expression of the inner mitochondrial membrane fusion protein Optic Atrophy 1 (Opa1). Mechanistically, the endoplasmic reticulum (ER) senses conditional and inducible skeletal muscle ablation of Opa1 and signals via the Unfolded Protein Response (UPR) a nuclear program that triggers muscle and adipose tissue loss. Conditional as well as inducible Opa1 deletion in muscle altered mitochondrial morphology and function but not DNA content, ultimately reducing growth and lifespan. Mechanistically, UPR activation mediated muscle stem cells loss, protein synthesis inhibition, ubiquitinproteasome activation and FGF21 expression leading to a fasting-like condition. Indeed, pharmacological inhibition of ER stress blunted the atrophy program and restored a normal muscle mass. Thus, the communication between mitochondria and ER controls muscle and systemic response to mitochondrial malfunction.

1. Romanello V, Sandri M. Mitochondrial biogenesis and fragmentation as regulators of protein degradation in striated muscles. J Mol Cell Cardiol. 2013;55:64-72. doi: 10.1016/j.yjmcc.2012.08.001. Epub 2012 Aug 10.

$$
* * * * *
$$

\section{Mitochondrial biogenesis and autophagy as targets for improved longevity}

\section{Christiaan Leeuwenburgh}

University of Florida, Institute on Aging, College of Medicine, Department of Aging and Geriatric Research, US. E-mail: "Leeuwenburgh,Christiaan" <cleeuwen@ufl.edu>

Dysfunctional mitochondria and insufficient autophagy are common in aged muscle and neuronal tissues. The mitochondrial theory of aging is based on the premise that cumulative damage caused by the production of oxidants can alter nuclear and mtDNA (e.g., point mutations and deletions). It is well established that defects in mtDNA lead 


\title{
CIR-Myo News: Abstracts of the 2015 Spring Padua Muscle Days
}

\author{
Terme Euganee Padua (Italy), March 12 - 14, 2015
}

\section{Abstracts}

to a decline in mtDNA abundance (content) and a reduced number of genes encoding mitochondrial proteins that are essential for the proper assembly and maintenance of mitochondria. Additionally, lower mitochondrial protein synthesis rates, disturbances in mitochondrial enzyme activities, altered import protein machinery, and lower oxidative capacity and ATP synthesis have been documented to occur in aged tissues. Besides being less bioenergetically efficient, damaged mitochondria also produce increased amounts of reactive oxygen species, specifically under stressed conditions (ischemia, reperfusion, environmental toxins). The age-related accumulation of dysfunctional mitochondrial likely results from the combination of impaired clearance of damaged organelles by autophagy and inadequate replenishment of the cellular mitochondrial pool by mitochondriogenesis. Muscle has been studied extensively, but less attention has been paid to the declining function of peripheral nerves with age. We recently showed in animals a drastic decline in the expression of glial and neuronal proteins in myelinated peripheral nerves with age, which is significantly ameliorated by lifelong calorie restriction. We also noted an improvement in nerve architecture with caloric restriction due to a sustained expression of protein chaperones, markers of the autophagylysosomal pathway and marked reduction in oxidative stress and inflammation. In muscle of humans, we recently examined whether mitochondrial regulation differed in muscle from elderly subjects classified as high- or low functioning, when compared to young subjects. Mitochondrial respiration rates, PGC- $1 \alpha$, a mitochondrial regulator, Sirt3, a mitochondrial deacetylase, the mitochondrial fusion protein Opa1, were all markedly suppressed in both high and low functioning subjects compared to healthy controls. In addition, we will present data related to a human study in which we show that a combination of diet and exercise has a beneficial effects on protein quality control, autophagy and mitochondrial biogenesis in muscle. This study was to assess the effects of a 6-month weight loss program combined with moderateintensity exercise on the cellular quality control mechanisms of autophagy and ubiquitin-proteasome, as well as mitochondrial function in the skeletal muscle of older obese women. Taken together, the age-related decline in functional molecules benefitting mitochondrial function (and impaired mitochondrial regulatory pathways) and autophagy (including alterations in other cellular protein homeostatic mechanisms), play a major role to cellular dysfunction with age and provide us with biological targets for intervention studies. Future studies should consider combining exercise with other metabolic and/or anabolic factors (i.e., testosterone, resveratrol, or other bioactive compounds or drugs) to enhance response on major parameters of biological and physical health compared to either regimen alone. Our goal is to provide additional validation to findings that exercise combined with natural and/or pharmaceutical compounds have the most powerful hormetic response to achieve optimal health.

1. Joseph, AM, Adhihetty PJ, Wawrzyniak et al. Dysregulation of mitochondrial quality control processes contribute to sarcopenia in a mouse model of premature aging. PloS one 2013;8:e69327. doi: 10.1371/journal. pone.0069327. Print 2013.

2. Marzetti E, Calvani R, Cesari M,et al. Mitochondrial dysfunction and sarcopenia of aging: From signaling pathways to clinical trials. Int $\mathbf{J}$ Biochem Cell Biol 2013;45:2288-301. doi: 10.1016/j.biocel.2013.06.024.

3. Joseph, AM, Adhihetty PJ, Buford TW, et al. The impact of aging on mitochondrial function and biogenesis pathways in skeletal muscle of sedentary high- and lowfunctioning elderly individuals. Aging cell 2012;11:8019. doi: 10.1111/j.1474-9726.2012.00844.x.

4. Wohlgemuth SE, Lees HA, Marzetti E, et al. An exploratory analysis of the effects of a weight loss plus exercise program on cellular quality control mechanisms in older overweight women. Rejuvenation Res 2011;14:315-24. doi: 10.1089/rej.2010.1132.

5. Wohlgemuth SE, Seo AY, Marzetti E, et al. Skeletal muscle autophagy and apoptosis during aging: effects of calorie restriction and life-long exercise. Exp Gerontol 2010;45:138-48. doi: 10.1016/j.exger.2009.11.002.

6. Kujoth GC, Hiona A, Pugh TD, et al. Mitochondrial DNA mutations, oxidative stress, and apoptosis in mammalian aging. Science 2005;309:481-4.

$$
* * * * *
$$

\section{The Mitochondrial Calcium Uniporter controls skeletal muscle trophism in vivo}

\section{Cristina Mammucari}

Department of Biomedical Sciences, University of Padova, Italy

E-mail: cristina.mammucari@unipd.it

Muscle atrophy contributes to the poor prognosis of many pathophysiological conditions, but pharmacological therapies are still limited. Muscle activity leads to major swings in mitochondrial $\left[\mathrm{Ca}^{2+}\right]$ which control aerobic metabolism, cell death and survival pathways. We have investigated in vivo the effects of mitochondrial $\mathrm{Ca}^{2+}$ homeostasis in skeletal muscle function and trophism, by overexpressing or silencing the Mitochondrial Calcium Uniporter (MCU). The results demonstrate that both in developing and in adult muscles MCU-dependent mitochondrial $\mathrm{Ca}^{2+}$ uptake has a marked trophic effect that does not depend on aerobic control, but impinges on two major hypertrophic pathways of skeletal muscle, PGC-1 $\alpha 4$ and IGF1-AKT/PKB. In addition, MCU overexpression protects from denervation-induced atrophy. These data reveal a novel $\mathrm{Ca}^{2+}$-dependent organelle-tonucleus signaling route, which links mitochondrial function to the control of muscle mass and may represent a possible pharmacological target in conditions of muscle loss.

1. Mammucari C, Gherardi, G, Zamparo, et al. (2015) The Mitochondrial Calcium Uniporter controls skeletal muscle trophism in vivo. Cell reports, in press.

2. Rizzuto R, De Stefani D, Raffaello A, Mammucari C. Mitochondria as sensors and regulators of calcium signalling. Nat Rev Mol Cell Biol 2012;13:566-78. doi: $10.1038 / \mathrm{nrm} 3412$. 


\title{
Eur J Transl Myol/Basic Appl Myol 2015; 25 (3): 145-182 \\ CIR-Myo News: Abstracts of the 2015 Spring Padua Muscle Days
}

\author{
Terme Euganee Padua (Italy), March 12 - 14, 2015
}

\section{Abstracts}

Keeping mitochondria in shape: a matter of life or death

Luca Scorrano

Department. of Biology and Dulbecco-Telethon Institute, Venetian Institute of Molecular Medicine, University of Padua, Italy

E-mail: luca.scorrano@unipd.it

Mitochondrial ultrastructural and morphological changes have been implied in the control of several physiological and pathological changes, including the progression of apoptosis. ${ }^{1}$ However, the precise role of mitochondrial dynamics in the response to reversible and irreversible cellular damage is not completely understood. Today we will present our recent data obtained in genetic models of ablation and up-regulation of the key mitochondrial shaping proteins Optic atrophy 1 (Opa1) and mitofusin in D. melanogaster and in the mouse. The in vivo experiments of tissue damage by inducing atrophy, apoptosis or ischemia/reperfusion indicate that the master cristae biogenetic regulator Opa1 can prevent multiple forms of tissue damage by controlling mitochondrial cytochrome c release and metabolic efficiency. On the other hand, the interplay between mitochondria and the endoplasmic reticulum is highlighted by the ablation of the multifunctional mitofusin of the fruitfly, that results in the development of ER stress that contributes to the lethality of the phenotype. Our data indicate that the shape of mitochondria dictates function of the organelle and therefore complex tissue responses, opening the possibility for treatment of pathological conditions where mitochondrial dysfunction plays a crucial role.

1. Kasahara A, Scorrano L. Mitochondria: from cell death executioners to regulators of cell differentiation. Trends Cell Biol 2014;2:761-70. doi: 10.1016/j.tcb.2014.08.005.

2. Marini ES, Giampietri $C$, Petrungaro $S$, et al.The endogenous caspase-8 inhibitor c-FLIPL regulates ER morphology and crosstalk with mitochondria. Cell Death Differ. 2014 Dec 12. doi: 10.1038/cdd.2014.197.

$* * * * *$

Link between malignant hyperthermia and environmental heat stroke: just a medical hypothesis?

Feliciano Protasi

CeSI - Center for Research on Ageing \& DNICS - Dept. Neuroscience, Imaging, and Clinical Sciences; University G. d'Annunzio of Chieti, Italy.

E-mail: fprotasi@unich.it

Background. EC coupling in muscle links the transverse (T)tubule depolarization to release of $\mathrm{Ca}^{2+}$ from the sarcoplasmic reticulum (SR). ${ }^{1,2}$ These membranes communicate in specialized intracellular junctions, i.e. calcium release units (CRUs), thanks to a cross-talk between voltage-dependent $\mathrm{C}^{2+}$ channels $\mathrm{CaV}_{1.1}$ (or dihydropyridine receptors, DHPRs) in the T-tubule and $\mathrm{Ca}^{2+}$ release channels, or ryanodine receptors type-1 (RYR1), in the SR. Mutations in the gene encoding for RYR1), the $\mathrm{SR} \mathrm{Ca}^{2+}$ release channel, underlie debilitating, life-threatening muscle disorders such as central core disease (CCD) and malignant hyperthermia $(\mathrm{MH}){ }^{3,4}$ To date, $\mathrm{MH}$ is only seen as a clinical syndrome in which genetically predisposed individuals respond to volatile anesthetics in the operating room with potentially lethal episodes characterized by elevations in body temperature and rhabdomyolysis of skeletal muscle fibers. However, virtually identical over-heating episodes have been reported in individuals also after exposure to environmental heat, physical exertion, or even during febrile illness. ${ }^{5}$ The life-threatening nature of $\mathrm{MH}$ and environmental heat stroke (EHS) underscore the critical need for a deeper mechanistic understanding of these syndromes and for the development of new and effective treatments. Specific Gaps of Knowledge. A) Mutations in RYR1 have been found in many, but not all, MH cases suggesting the potential involvement of additional genes in the pathogenesis of this syndrome. B) The relationship between classic $\mathrm{MH}$ and over-heating episodes triggered by different stressors (heat, exertion, fever, etc.) is not yet widely recognized. C) The cascade of molecular mechanisms that from $\mathrm{SR} \mathrm{Ca}^{2+}$ leak leads to rhabdomyolysis of muscle fibers are still unclear and needs to be fully elucidated.

Recent breakthroughs. In the last years, thanks to the support of Telethon (GGP08153 and GGP13213), we have moved significant steps forward demonstrating in animal models that: A) MH episodes can result not only from mutations in RYR1, but also from mutations in proteins that interact with RYR1 (such as Calsequestrin-1, CASQ1); B) the mechanisms underlying hyperthermic episodes triggered by anesthetics and by heat/exertion are virtually identical, suggesting that these syndromes could be possibly treated/prevented using similar treatments; C) during lethal MH/EHS crises $\mathrm{Ca}^{2+}$ leak from intracellular stores results in a feed-forward mechanism mediated by excessive production of oxidative species of oxygen and nitrogen (ROS and RNS), which eventually will lead to depletion of the SR and to massive activation of Store Operated $\mathrm{Ca}^{2+}$ Entry (SOCE).

1. Schneider MF. Control of calcium release in functioning skeletal muscle fibers. Annu Rev Physiol 1994;56:46384.

2. Franzini-Armstrong C, Protasi F. Ryanodine receptors of striated muscles: a complex channel capable of multiple interactions. Physiol Rev 1997;77:699-729.

3. Jungbluth H. Central Core Disease. Orphanet J Rare Dis 2007;2:25-30.

4. Denborough M. Malignant hyperthermia. Lancet 1998;352:1131-6.

5. Lehmann-Horn F, Klingler W, Jurkat-Rott KNonanesthetic malignant hyperthermia. Anesthesiology 2011;115:915-7.

\section{Efficiency of electric neurostimulation in treatment of presbyphonia in an animal model}

Markus Gugatschka (1), Sophie Bachna-Rotter (1), Claus Gerstenberger (1), Jonathan Jarvis (2), Hans-Jörg Schlager (1), Gerhard Friedrich (1)

(1) ENT University Hospital Graz, Dept. of Phoniatrics, Medical University Graz, Austria; (2) John Moores University, Liverpool, UK

E-mail: Markus.Gugatschka@klinikum-graz.at

Age related changes of the muscle and its adjacent structures also affect the larynx. This is not only important for speech 


\section{CIR-Myo News: Abstracts of the 2015 Spring Padua Muscle Days}

Terme Euganee Padua (Italy), March 12 - 14, 2015

\section{Abstracts}

production, but plays a significant role for swallowing function too. Chronic electrical stimulation of the afferent nerve (recurrent laryngeal nerve) is a completely new therapeutic option that has not been tested before. 18 male Wistar rats were implanted with a unilateral nerve stimulator. Stimulation period was 8 weeks in all animals, twice daily. Changes were observed on the muscular level (cross section area, fiber size) as well as (immuno)-histochemically (e.g. myosin-heavy chain distribution). Compared to control group, chronical stimulation lead to changes in the parameters mentioned above. In conclusion, chronic electrical stimulation can be a new treatment option for age related changes of the larynx. The findings need to be proven in larger animals before going into human studies.

1. McMullen CA, Butterfield TA, Dietrich M, et al. Chronic stimulation-induced changes in the rodent thyroarytenoid muscle. J Speech Lang Hear Res 2011;54:845-53.

2. Shiotani A, Nakagawa H, Flint PW. Modulation of myosin heavy chains in rat laryngeal muscle. Laryngoscope. 2001;111:472-7.

3. Suzuki T, Connor NP, Lee K, et al. Age-related alterations in myosin heavy chain isoforms in rat intrinsic laryngeal muscles. Ann Otol Rhinol Laryngol. 2002;111:962-7

$$
* * * * *
$$

\section{Generation of 3D-models of laryngeal structures with special attention to the intrinsic laryngeal muscles}

Claus Gerstenberger, Hans-Jörg Schlager, SophieBachnaRotter, Matthias Graupp, Elke Fröhlich-Sorger, Karl Kiesler, Gerhard Friedrich, Marcus Gugatschka

ENT University Hospital Graz, Department of Phoniatrics, Medical University Graz, Austria

E-mail: Claus.Gerstenberger@klinikum-graz.at

The access to different structures in the larynx - especially to the intrinsic muscles in vivo - is limited. Additionally the volumetric quantification is problematic due to their covering with mucosa. Nevertheless it is necessary to generate accurate models of these structures for the purpose of answering muscle-specific issues. Nowadays this is possible with modern imaging procedures such as micro-CT scanning. This technology has advantages over MRI in terms of better resolution and the samples are not destroyed during the imaging process as in histologic sampling. To differentiate the muscles from the soft tissue, the samples are fixed and preserved in neutral buffered formalin $(\mathrm{NBF})$ and stained with iodine to enhance contrast in the CT-scan. The purpose of this study is to generate a 3D-model of the skeleton and the intrinsic laryngeal muscles with the segmentation and finite-element generation and 3D-analysis-software Amira ${ }^{\circledR}$. This modeling technique will be used in further experiments in the field of muscle stimulation for analyzing of the results, especially muscle volume and structure.

1. Jeffery NS, Stephenson RS, Gallagher JA,et al. Microcomputed tomography with iodine staining resolves the arrangement of muscle fibres. J Biomech 2011;44:18992.

2. Bakhshaee H, Moro C, Kost K, Mongeau L. ThreeDimensional Reconstruction of Human Vocal Folds and
Standard Laryngeal Cartilages Using Computed Tomography Scan Data. J Voice 2013;27:769-77.

3. Inagi K, Schultz E, Ford CN. An anatomic study of the rat larynx: Establishing the rat model for neuromuscular function. Otolaryngol Head Neck Surg 1998;118:74-81.

$* * * * *$

Laryngeal pacing for the treatment of bilateral vocal fold paralysis: preliminary long-term results of a prospective first-in-human study

Werner Lindenthaler (1), Andreas Harald Mueller (2), Rudolf Hagen (3), Gerhard Foerster (2), Wilma Harnisch, (3) Katrin Baumbusch (4), Claus Pototschnig (4)

(1) Med-El medical electronics, Innsbruck, Austria; (2) Department of Otorhinolaryngology, SRH Wald-Klinikum Gera, Gera, Germany; (3) Department of Otorhinolaryngology, University of Würzburg, Würzburg, Germany; (4) Department of Otorhinolaryngology, Innsbruck University Hospital, Innsbruck, Austria

E-mail: werner.lindenthaler@medel.com

Bilateral vocal fold paralysis (BVFP) is a potentially lifethreatening medical condition, which causes persistent dyspnoeic symptoms and has a significant impact on the patient's quality of life. Endoscopic enlargement techniques have been the standard treatment for BVFP for decades. ${ }^{1,2}$ Laryngeal pacing is a potential treatment based on the electrostimulation of the posterior cricoarytenoid (PCA) muscle. The data herein present the results of the long term performance of a new Laryngeal Pacemaker (LP) System implanted for 24-months in one patient. In a prospective multicentre study, 9 patients were implanted unilaterally with the LP System. 2 left the study prematurely. Of the 7 patients who completed the study, 1 has been implanted for 24months. Respiration quality; swallowing capacity; quality of life (SF-36 and GBI); six minute walk test (6MWT); jitter; maximum phonation time (MPT); voice range profile (VRP); Dysphonia Severity Index (DSI); Voice Handicap Index-12 (VHI-12); roughness, breathiness and hoarseness (RBH) were evaluated pre-operatively, 1-, 6-, 12-, and 24-months after implantation. Results: Respiratory, voice and life quality generally improved 6-months after implantation and remained stable between 6- and 24-months postimplantation. The results of VHI-12, MPT, DSI, Peak Expiratory Flow (PEF) and Peak Inspiratory Flow (PIF) further improved between 6- and 24-months after implantation. Swallowing quality remained unchanged. These preliminary results showed that the LP System is effective in reducing the symptoms of the BVFP in both the middle- and long-term. In particular, the implantation of the LP System correlated with improved respiratory, voice and life quality. Improvements were stable or increased further between 6- and 24-months after implantation. Evaluation after 24-months of follow-up of all the other patients of the study and larger cohort studies are planned to confirm these initial findings.

1. Mueller AH. Laryngeal pacing for bilateral vocal fold immobility. Curr Opin Otolaryngol Head Neck Surg 2011;19:439-43. 


\title{
CIR-Myo News: Abstracts of the 2015 Spring Padua Muscle Days
}

\author{
Terme Euganee Padua (Italy), March 12 - 14, 2015
}

\section{Abstracts}

2. Forster G, Arnold D, Bischoff SJ, et al. Laryngeal pacing in minipigs: in vivo test of a new minimal invasive transcricoidal electrode insertion method for functional electrical stimulation of the PCA. Eur Arch Otorhinolaryngol 2013;270:225-31.

3. Mueller AH. Laryngeal Neuroprothesis. ENT \& Audiology News 2012. $* * * * *$

\section{Optimized stimulation patterns for increased efficacy of muscle activation \\ Paolo Cavallari}

Section Human Physiology - DePT, Università degli Studi di Milano, Italy

E-mail: paolo.cavallari@unimi.it

A unit's "optimal pulse sequence" does not depend on the number of pulses in the train. This property ensures that a motor unit will always develop maximum tension-time area per pulse without a priori knowledge of how long its discharge burst will be, as long as its spikes are generated in time with a pattern corresponding to its optimal pulse sequence. Optimal pulse sequences always began with one or two short interpulse intervals. To produce the repetitive, submaximal, short duration muscle contractions with a rapid rate of force production that are needed during many of the movement patterns elicited during FES, high frequency, moderate-intensity, short-duration trains could be used. These trains have the disadvantage of producing a rapid rate of fatigue of muscle tension. Lower-frequency trains (e.g., 20 pps) produce much less fatigue, but produce a much slower rate of rise of force than higher-frequency trains. Properties of electrical stimulus pulses, patterns and trains producing maximum force, tension, minimum fatigue, to reduce the level of stimulus pain during high levels of muscle stimulation or reducing electrical energy for powering the stimulation will be presented.

$$
* * * * *
$$

\section{Neuromodulation with implanted devices: models of resistance exercise in rodent limbs}

Martin Schmoll, Ewald Unger, Martin Bijak, Herman Lanmueller, Jonathan C. Jarvis

(1) Liverpool John Moores University, UK; (2) Medical University of Vienna, Austria

E-mail: "Jarvis, Jonathan" <J.C.Jarvis@1jmu.ac.uk>

Much has been learned about the response of muscle to changes in activity by means of implantable neuromodulators. $^{1-3}$ The pattern of daily activity of a particular muscle group can be reliably changed over periods of weeks, and in many cases, an internal control (for example an unstimulated contralateral limb) is available. One limitation of such studies is that the load on the stimulated muscle is more difficult to control than the activation and thus experiments on resistance exercise in small animals have relied on methods of weight lifting that require specific training or repetitive anaesthesia. ${ }^{4}$ We have investigated in experiments under anaesthesia the possibility of loading the dorsiflexors of the foot by co-activation of the plantarflexors.
We have made preliminary experiments to determine the difference in force produced in the tibialis anterior tendon with: 1) unloaded contractions (common peroneal stimulation, foot free to move), 2) isometric contractions (common peroneal stimulation, foot held externally) and 3) co-contractions (common peroneal stimulation with spillover stimulation of the tibial nerve to recruit plantarflexion). We have used a new design of in-line load cell to report the force experienced by the tibialis anterior tendon. We can begin to give numerical values to the difference in force experienced by the musculoskeletal system when movement is restricted. This will allow us to include not only the activity pattern but also the loading pattern in our analysis of changes in muscle due to increased activity.

1. Vickerton P, Jarvis JC, Gallagher JA, et al. Morphological and histological adaptation of muscle and bone to loading induced by repetitive activation of muscle. Proc Biol Sci 2014 Aug 7;281(1788):20140786. doi: 10.1098/rspb.2014.0786.

2 Ashley Z, Sutherland H, Russold MF, et al.Therapeutic stimulation of denervated muscles: the influence of pattern. Muscle Nerve 2008;38:875-86.

3. Sutherland H, Jarvis JC, Salmons S. Pattern dependence in the stimulation-induced type transformation of rabbit fast skeletal muscle. Neuromodulation. 2003;6:176-89.

4. Wong TS, Booth FW. Skeletal muscle enlargement with weight-lifting exercise by rats. J Appl Physiol 1988;65:950-4.

\section{Facial nerve pacing to restore eye blink in acute facial palsy, act II}

Paolo Cavallari (1), Alice Frigerio (1), Tessa Hadlock (2), Chris Knox (2), Marc Hohman (2), James Heaton (2)

(1) Section Human Physiology - DePT, Università degli Studi di Milano, Italy; (2) Facial Nerve Center, MEEI, Harvard Medical School

E-mail: paolo.cavallari@unimi.it

Facial nerve pacing may restore eye blink, and to a lesser degree facial expression, using electrical stimulatory techniques. The feasibility of facial pacing via functional electrical stimulation (FES) has already been assessed in animal models (e.g. rabbit and dog) and healthy human volunteers. We performed a feasibility study of whether eye blink can be elicited by transcutaneous electrical nerve stimulation in patients with acute facial palsy, and obtained real-time sensation feedback from participants to determine whether stimulation would be tolerable for daily eye blink restoration. Methods: A cohort of 9 individuals (4 males and 5 females) experiencing paralysis of orbicularis oculi muscle were enrolled at 6-42 days from onset. Unilateral stimulation of zygomatic facial nerve branches to elicit eye blink via orbicularis oculi contraction was achieved with brief bipolar constant-current pulse trains, delivered transcutaneously by epicutaneous electrode placement. Stimulation trains patterns fell in the range of $0.2-1.2 \mathrm{~ms}$ pulse duration, $150-250 \mathrm{~Hz}$ pulse frequency, and 1-15 mA pulse amplitude. The relationship between stimulation parameters and cutaneous sensation was obtained using the the Wong-Baker Faces Pain Rating Scale in a continuous manner throughout stimulation 


\title{
Eur J Transl Myol/Basic Appl Myol 2015; 25 (3): 145-182 \\ CIR-Myo News: Abstracts of the 2015 Spring Padua Muscle Days
}

\author{
Terme Euganee Padua (Italy), March 12 - 14, 2015
}

\section{Abstracts}

trials. By studying an initial 9 individuals, we established descriptive statistics regarding the average stimulation thresholds for initial twitch, complete closure, and the relationship between those thresholds and the corresponding level of stimulation discomfort (Wong-Baker Pain Rating Scale scores). In conclusion, facial nerve pacing can potentially restore eye blinks in individuals with acute facial paralysis. Despite variability in reported levels of stimulation-related discomfort, effective pulse trains could be delivered at tolerable current levels. These patients would benefit from a biomimetic device to facilitate eye closure during waking hours in the weeks between paralysis onset and the return of normal blink.

$$
* * * * *
$$

\section{Implantable neuromuscular stimulator for small animals}

Hermann Lanmüller(1), Manfred Bijak(1), Martin Schmoll(2), Michael Haller(1), Ewald Unger(1), Jonathan C. Jarvis (2)

(1) Medical University of Vienna, Austria, (2) Liverpool John Moores University, UK

E-mail: Hermann.Lanmueller@ meduniwien.ac.at

Neuromuscular basic research is mainly done in small laboratory animals, particularly in mice and rats. Long term electrical activation of investigated muscles can only be reliably done when implantable pulse generators are used. A major criterion for such implants is a small volume. The applied daily stimulation patterns depend strongly on the research goal and can be rather different. Consequently, highly adjustable pattern generation and easy handling are essential. The presented implantable neuromuscular stimulator, called MiniStim fulfills these requirements.

The MiniStim is powered by a single Lithium primary cell and generates monophasic constant current pulses followed by a charge balancing exponential reverse current. The amplitude can be set with a resolution of 8 bit up to $2 \mathrm{~mA}$. The minimum pulse duration is $130 \mathrm{us}$ and can be increased in steps of 130us. All pulse parameters and stimulation sequence parameters are stored and controlled by a microcontroller. Two different stimulator types have been developed and tested up till now. ${ }^{1}$ MiniStim type A is preprogrammed during the manufacturing process. Different stimulation patterns can be selected by the use of a permanent magnet. Each touch close to the pulse generator is detected by the internal reed switch and advances to the next in the pre-programmed palette of patterns. Ministim A is intended for experimental studies, which do not require adjustment of stimulation parameters during a period of programmed activity. MiniStim type B can be programmed freely by a bidirectional RF link between a programming tablet and the implanted device. Stimulation data are designed or modified on an Android based tablet computer, which is linked by Bluetooth to the programmer device. Amplitude shift keying is used for data transfer from programmer to the pulse generator and pulse shift keying for transmission in the opposite direction, all operating on a carrier frequency of $400 \mathrm{kHz}$. The transmission link works reliably within an axial and radial displacement of up to $40 \mathrm{~mm}$. Epoxy resin or silicone rubber are used for implant encapsulation. Both implant types have been tested successfully in rats for a period of up to 2 months. The implemented stimulation patterns differ markedly and range from 24 bursts daily up to 1452 bursts. There is therefore the possibility to test the effect of complex patterns of stimulation such as those made up of intensive bursts of activity separated by variable rest periods.

1. Jarvis J, Bijak M, Lanmüller $H$. et. al. New Vienna/Liverpool implantable stimulator. Eur $\mathrm{J}$ Trans Myol 2013;23:184.

$$
* * * * *
$$

\section{The Vienna Liverpool mini neuromodulator: improved lifetime, user interface and versatility}

Martin Schmoll (1), Manfred Bijak (2), Ewald Unger (2), Jonathan C. Jarvis (1), Hermann Lanmueller (2)

(1) Liverpool John Moores University, UK; (2) Medical University of Vienna, Austria,

E-mail: "Jarvis, Jonathan" <J.C.Jarvis@ljmu.ac.uk>

The Vienna/Liverpool implantable stimulator is the result of many years of development taking advantage of the steady progress in miniaturisation and gate density of integrated circuits. Functions such as digital to analogue conversion and amplification that formerly required the use of additional chips are now packaged into very small plastic packages that are suitable for experimental implants. We have demonstrated that there is sufficient processing power now for a device that is remotely programmable via a short range radio frequency link that allows complete specification of amplitude and pattern a stimulation regime at any stage of an experiment.

1. Jarvis $J$, Bijak $M$, Lanmüller $H$. et. al. New Vienna/Liverpool implantable stimulator. Eur $\mathbf{J}$ Trans Myol 2013;23:184.

$$
* * * * *
$$

Daily electrical muscle stimulation enhances functional recovery and upregulates Muscular Brain Derived Neurotrophic Factor (BDNF) following nerve transection and repair in rats

Michael P. Willand (1), Jennifer J. Zhang (1), Cameron Chiang (1), Elyse Rosa (2), Stephen W.P. Kemp (1), Margaret Fahnestock (2), Gregory Borschel (1), Tessa Gordon (1)

(1) Division of Plastic and Reconstructive Surgery, The Hospital for Sick Children, Toronto, ON, Canada. (2) Department of Psychiatry \& Behavioural Neurosciences, McMaster University, Hamilton, ON, Canada.

E-mail: mike.willand@gmail.com

The use of chronic electrical muscle stimulation for treating partially or completely denervated muscle is controversial. Recently, we used a daily electrical muscle stimulation paradigm over a two week period after nerve injury and immediate repair. We showed that muscle stimulation significantly increases the number of motor nerves reinnervating muscles and axon outgrowth within the distal nerve stump. ${ }^{1}$ Activity-dependent intramuscular trophic factor release acts on regenerating axons, which may explain the increased early regeneration in stimulated muscle. 


\title{
CIR-Myo News: Abstracts of the 2015 Spring Padua Muscle Days
}

\author{
Terme Euganee Padua (Italy), March 12 - 14, 2015
}

\section{Abstracts}

However, chronic electrical muscle stimulation applied throughout the entire reinnervation period has not been previously assessed. In the present study we hypothesized that stimulation would enhance functional recovery over three months and that stimulation enhances early intramuscular trophic factor release. Six groups of Thy1-GFP transgenic male rats underwent tibial nerve transection and immediate repair using two epineurial sutures. One group of rats underwent daily electrical muscle stimulation of the gastrocnemius with a paradigm producing 600 equally separated contractions throughout one hour, delivered 5 days per week. Rat gastrocnemius muscles were electrically stimulated for 1, 2, or 3 months and then we assessed muscle force, contractile properties, motor unit numbers, and wet muscle weight. Rats in the 3 month group were serially evaluated using a tapered beam test to evaluate skilled locomotion. Muscles underwent immunohistological examination of motor end plate reinnervation. Two additional groups of rats were subjected to the same nerve injury and were used to investigate early intramuscular trophic factor release following two weeks of electrical muscle stimulation or no treatment. The number of motor units was significantly increased after daily muscle stimulation for all three time points $(1,2$, and 3 months). Mean motor unit sizes were significantly smaller in stimulated muscles, suggesting that muscle stimulation may inhibit terminal sprouting as reported by others. This may allow for a more natural course of reinnervation resulting in improved functional recovery. Indeed, skilled locomotion tests showed that stimulated muscles enhanced and maintained recovery at levels no different than normal functioning rats, whereas nonstimulated controls became progressively worse and did not recover to baseline. After two weeks of stimulation, BDNF was significantly upregulated in stimulated muscle compared to non-stimulated muscle. In conclusions, treatment of denervated muscle using electrical stimulation significantly enhanced muscle reinnervation, and upregulation of BDNF may explain this enhancement. As the muscle continues to reinnervate, tailoring the stimulation paradigm to improve muscle force and fatigability may further enhance muscle recovery.

1. Willand MP, Chiang CD, Zhang JJ, Kemp SW, Borschel GH, Gordon T. Daily Electrical Muscle Stimulation Enhances Functional Recovery Following Nerve Transection and Repair in Rats. Neurorehabil Neural Repair. 2014 Dec 11. pii: 1545968314562117.

$* * * * *$

\section{CAPs in an isolated sciatic nerve of a rat elicited with quasitrapezoidal stimulating pulses}

Janez Rozman (1,2), Polona Pečlin (1), Monika C. Žužek (3), Milka Vrecl (3), Robert Frangež (3)

(1) Center for Implantable Technology and Sensors, ITIS d. o. o. Ljubljana; (2) Institute of Pathophysiology, Medical Faculty, University of Ljubljana; (3) Institute of Physiology, Pharmacology and Toxicology, Veterinary Faculty, University of Ljubljana, Republic of Slovenia

E-mail: "Janez Rozman" <jnzrzmn6@gmail.com>
The method commonly used in FES is in general a nonselective nerve stimulation, which in turn causes frequent occurrence of undesirable side effects. To alleviate such problems, various models and electrode systems that selectively stimulate certain features have been developed. In this regard, we review the functional performance of quasitrapezoidal current biphasic stimulating pulse for fibertype selective nerve stimulation. The stimulus should predominantly stimulate myelinated $A \beta$-fibres, minimize the stimulation of the myelinated $A \alpha$-fibers and $A \delta$-fibres, and bypass the stimulation of the non-myelinated $\mathrm{C}$-fibres. The study including nerve conduction velocity and compound action potential (CAP) measurements is performed on an isolated segment of a rat sciatic nerve. A stimulus is applied to the nerve using a pair of hook platinum wire electrodes while CAP is measured at the two sites along the nerve using two couples of identical recording hook platinum electrodes. Positive recording electrodes of the two couples, are situated at the distance 9.6 for the first couple and $19.2 \mathrm{~mm}$ for the seconfd couple from the stimulating cathode. Results of the study show, that ascending parameters of the stimulus, namely, ic - intensity of the cathodic phase, tc - width of the cathodic phase, texp - width of the cathodic exponential decay and $\tau \exp$ - time constant of the cathodic exponential decay, influences the peak values and all the temporal parameters of the CAP1 and CAP2. Results also show that a peak value of the CAP1max - CAP1 maximum and CAP2max - CAP2 maximum can be obtained when parameters of the stimulus are set within the following range: $\mathrm{ic}=2.5$ to $3.35 \mathrm{~mA}, \tau \exp =330-440 \mu \mathrm{s}, \mathrm{tc}=325-430 \mu \mathrm{s}$ and texp $=330-440 \mu \mathrm{s}$, respectively. The most important finding of the present study however, is that when certain parameters of the stimulus waveform are selected, the contribution of the myelinated $A \alpha$-fibers and $A \delta$-fibres can be minimized, the contribution of the non-myelinated $\mathrm{C}$-fibres bypassed and the contribution of the myelinated $\mathrm{A} \beta$-fibres actualized. The confirmation of this statement can be found in the measured CAP1 and CAP2, where in spite of the three aforementioned types of nerve fibers are present within the nerve, only one peak in both the measured CAP1 and CAP2 is observed. It was also shown that only tc slightly shorter and slightly larger than $365 \mu \mathrm{s}$ and texp slightly shorter and slightly larger than $270 \mu \mathrm{s}$ are appropriate to be used with the predefined stimulus waveform and arrangement of stimulating and recording electrodes within this particular stimulating/recording setup. It can be concluded that the design of stimulating electrodes and stimuli waveform based on the obtained results, could act as a useful tool for nerve stimulating electrodes development that potentially enable fibre-type selective stimulation of nerve fibers.

1. Butson CR, Miller IO, Normann RA, Clark GA. Selective neural activation in a histologically derived model of peripheral nerve. J Neural Eng 2011;8:036009. doi: $\quad 10.1088 / 1741-2560 / 8 / 3 / 036009$. S17412560(11)74580-8

2. Gu D, Gander RE, Crichlow EC. Determination of nerve conduction velocity distribution from sampled compound action potentials. IEEE Transactions on Biomedical Engineering 1996;43: 829-38.

3. Oğuzhanoğlu A, Erdoğan C, Tabak E, Cenikli U. Comparison of conduction velocities of nerve fibers to 


\title{
Eur J Transl Myol/Basic Appl Myol 2015; 25 (3): 145-182 \\ CIR-Myo News: Abstracts of the 2015 Spring Padua Muscle Days
}

\author{
Terme Euganee Padua (Italy), March 12 - 14, 2015
}

\section{Abstracts}

smaller and larger muscles in rats. Int $\mathrm{J}$ Neurosci 2010;120: 76-9. (doi: 10.3109/00207450903389370.

$$
* * * * *
$$

\section{Resistance training and skeletal muscle: a healthy virtuous relationship}

\section{Antonio Paoli}

Nutrition and Exercise Physiology Laboratory, Department of Biomedical Sciences, University of Padova, Italy E-mail: antonio.paoli@unipd.it

Adaptive changes of muscle fibers can occur in response to variations in the pattern of neural stimulation, loading conditions, availability of substrates, and hormonal signals These signals stimulate pathways which may lead to changes in fiber size and/or fiber type. Fiber size is in a dynamic equilibrium between protein accumulation (hypertrophy) and protein loss (atrophy). The paradigmatic human models for muscle hypertrophy is resistance training (RT). But RT can influences not only muscle mass but also many other metabolic pathways that may affect positively health. Recently our group has demonstrated the different effects of circuit training carried out at low or high intensity on some anthropometric and metabolic variables. One of the major issue regarding the effects of RT on muscle mass is the extreme complexity of such kind of exercise. Resistance training may be carried out via different methods that have been shown to have differing effects on muscle metabolism and signalling pathways. As a matter of fact a resistance training program is a composite of several important variables including: 1) muscle action used, 2) type of resistance used, 3) volume (total number of sets and repetitions), 4) exercises selected and workout structure (e.g., the number of muscle groups trained), 5) the sequence of exercise performance, 6) rest intervals between sets, 7) repetition velocity and 8) training frequency that could be taken into account. For this reason we have investigated the effects of two different kind of RT on muscle signalling. 12 healthy and physically active subjects performed in two different moments and with different legs an high intensity resistance training (HIRT) and traditional resistance training (TRT). HIRT consisted in 2 sets at the leg extension performed with the following technique: 6 repetitions, 20 seconds rest, $2 / 3$ repetitions, 20 secs rest, $2 / 3$ repetitions with 2 min 30 secs rest between the sets. TRT consisted of 4 sets of 15 repetitions with 1 min 15 secs of rests between the sets. Biopsies from the vastus lateralis were taken one week before training $(\mathrm{Tb})$ sessions, immediately after training (T0), 6 hours after (T6) and 24 hours after (T24). Western blot analysis was performed to investigate mTOR, Akt, 4EBP1, S6, AMPK and ACC. Our results showed that different RT execution affects muscle pathways in a specific manner. These results suggest that a specific kind of RT should be studied to counteract atrophy mechanism related to disuse or ageing.

1. Patti A, Bianco A, Paoli A, et al. Effects of pilates exercise programs in people with chronic low back pain: a systematic review. Medicine (Baltimore). 2015 Jan;94(4):e383. doi: 10.1097/MD.0000000000000383.

$$
* * * * *
$$

The contribution of stem cell therapy to skeletal muscle remodeling in heart failure

Giorgio Vescovo (1), Chiara Castellani (2), Barbara Ravara (3), Chiara Franzin (4), Michela Pozzobon (4), Regina Tavano (3), Luisa Gorza (3), Emanuele Papini (3), Roberto Vettor (5), Gaetano Thiene (2), Annalisa Angelini (2)

(1) Internal Medicine, Sant Antonio Hospital, Padova; (2) Department of Cardiac, Thoracic and Vascular Sciences, University of Padua; (3) Department of Biomedical Sciences, University of Padua; (4) Fondazione Città della Speranza, Woman and Child Health Department, Stem Cells and Regenerative Medicine Lab, University of Padua; (5) Department of Medicine-Dimed, University of Padua, Italy E-mail: " gvescovo" <gvescovo@yahoo.it>

The aim of our study was to investigate whether stem cell (SC) therapy with human amniotic fluid stem cells (hAFS, fetal stem cells) and rat adipose tissue stromal vascular fraction cells-GFP positive cells (rSVC-GFP) was able to produce favorable effects on skeletal muscle (SM) remodeling in a well-established rat model of right heart failure (RHF). RHF was induced by monocrotaline (MCT) in Sprague-Dawley rats. Three weeks later, four millions of hAFS or rSVC-GFP cellswere injected via tail vein. SMremodeling was assessed by Soleus muscle fiber cross sectional area (CSA), myocyte apoptosis,myosin heavy chain (MHC) composition, satellite cells pattern, and SC immunohistochemistry. hAFS and rSVC-GFP injection produced significant SC homing in Soleus $(0.68 \pm 1.0$ and $0.67 \pm 0.75 \%$ respectively), with a $50 \%$ differentiation toward smoothmuscle and endothelial cells. Pro-inflammatory cytokines were down regulated to levels similar to those of controls. SC-treated (SCT) rats showed increased $\mathrm{CSA}(\mathrm{pb} 0.004$ vsMCT) similarly to controlswith a reshift toward the slow MHC1 isoform. Apoptosis was significantly decreased $(11.12 . \pm 8.8$ cells $/ \mathrm{mm} 3 \mathrm{hAFS}$ and $13.1+7.6 \mathrm{rSVC}$ GFP) (pb0.001 vs MCT) and similar to controls (5.38 \pm 3.0 cells $/ \mathrm{mm} 3)$. RHF rats showed a dramatic reduction of satellite cells(MCT $0.2 \pm 0.06 \%$ Pax7 native vs controls $2.60 \pm 2.46 \%$, pb0.001), while SCT induced a repopulation of both native and SC derived satellite cells (pb0.005). In conclusion, SC treatment led to SM remodeling with satellite cell repopulation, decreased atrophy and apoptosis. Modulation of the cytokine milieu might play a crucial pathophysiological role with a possible scenario for autologous transplantation of SC in pts with CHF myopathy.

\section{Multicomponent interventions against sarcopenia and frailty}

\section{Emanuele Marzetti}

Department of Geriatrics, Neurosciences and Orthopedics, Catholic University of the Sacred Heart School of Medicine, Teaching Hospital "Agostino Gemelli", Italy E-mail: "Emanuele Marzetti" <emarzetti@live.com>

The existing healthcare systems built around the traditional paradigm of patients suffering from a single acute illness are largely unprepared to face the increasing demands for health services that can specifically address the medical needs of 


\title{
CIR-Myo News: Abstracts of the 2015 Spring Padua Muscle Days
}

\author{
Terme Euganee Padua (Italy), March 12 - 14, 2015
}

\section{Abstracts}

older, multimorbid people. As a consequence, a large and growing segment of the older European population is currently suffering from medical conditions that cannot be efficiently managed by the available healthcare services. ${ }^{1}$ In this scenario, the geriatric syndrome of frailty plays a major role. Frailty is defined as a multidimensional condition characterised by decreased reserve and diminished resistance to stressors. ${ }^{2}$ Such extreme vulnerability exposes the older individual at an increased risk of morbidity, disability, inappropriate healthcare use, institutionalization, poor quality of life, and mortality. ${ }^{2}$ Detecting and contrasting frailty are thus crucial for impeding the progression of the syndrome, preventing its detrimental clinical consequences, and ensuring the sustainability of healthcare systems. Unfortunately, to date, no healthcare programs or pharmacological treatments are available for frail older people, largely because of the lack of a precise, universal definition of the condition. Such a barrier may be overcome by developing and validating a robust conceptual framework to achieve a practical operationalisation of frailty. The recognition of sarcopenia as a central component of physical frailty may allow overcoming the existing uncertainties in the field, while providing a biological substrate for preventive and therapeutic interventions. ${ }^{3}$ In this regard, it should be noted that monodimensional interventions may be insufficient at reversing the complex frailty status. Conversely, multi-component interventions have shown to be particularly useful when dealing with age-related syndromic conditions. ${ }^{4}$ Indeed, the simultaneous targeting of multiple and heterogeneous mechanisms underlying the disabling cascade may enhance the intervention effects. ${ }^{5}$ At the same time, multi-component interventions resemble what is commonly done in usual clinical practice, in which the intervention is designed around the needs and resources of the individual. Based on the existing evidence, it is expected that the combination of physical exercise and nutrition, with the eventual support of appropriate e-health services, may provide the greatest benefits in the management of physical frailty and sarcopenia.

1. Bien B, McKee KJ, Dohner H, Triantafillou J, Lamura G, Doroszkiewicz H, Krevers B, Kofahl C. Disabled older people's use of health and social care services and their unmet care needs in six European countries. Eur J Public Health. 2013;23:1032-8.

2. Fried LP, Tangen CM, Walston J, Newman AB, Hirsch C, Gottdiener J, Seeman T, Tracy R, Kop WJ, Burke G, McBurnie MA. Frailty in older adults: evidence for a phenotype. J Gerontol A Biol Sci Med Sci. 2001;56:M146-M156.

3. Marzetti E, Leeuwenburgh C. Skeletal muscle apoptosis, sarcopenia and frailty at old age. Exp Gerontol. 2006;41:1234-8.

4. Newman AB, Cauley JA. The epidemiology of aging. New York: Springer Dordrecht Heidelberg; 2012.

5. Cesari M, Landi F, Vellas B, Bernabei R, Marzetti E. Sarcopenia and physical frailty: two sides of the same coin. Front Aging Neurosci. 2014;6:192.
Role of mitochondrial DNA-TFAM interactions in the age-related mitochondrial dysfunction

\author{
Angela Maria Serena Lezza
}

Department. of Biosciences, Biotechnologies, and Biopharmaceutics, University of Bari, Italy

E-mail: angelamariaserena.lezza@uniba.it

Aging involves the progressive functional decline of tissues, which includes the dysfunction of the mitochondrial respiratory complexes leading to a reduced adenosine triphosphate (ATP) synthesis and to a decreased cell bioenergetics capability. Mitochondrial Transcription Factor A (TFAM) is regarded as a histone-like protein of mitochondrial DNA (mtDNA), performing multiple functions for this genome. Due to the close connection between mtDNA transcription and replication, TFAM has been supposed to participate to the regulation of mtDNA copy number. TFAM is also involved in the constitution of mtDNA nucleoids and might be part of a system responsible for sensing and repair of oxidative damage to mtDNA. Because of the relevant involvement in the regulation of mitochondrial biogenesis and transcription, TFAM expression has been investigated in tissues such as the brain, heart and skeletal muscle that have a high dependence on oxidative metabolism either in metabolically very active tissues, such as the liver. Aging affects mitochondria in a tissue-specific manner and so far only calorie restriction (CR) is able to delay or prevent the onset of several age-related alterations also in mitochondria. Age-related changes in mtDNA content were reported in various tissues. We detected TFAM amount, TFAM-binding to mtDNA and mtDNA content in three aged rat tissues. Samples of the frontal cortex (1) and soleus skeletal muscle (2) from 6- and 26-month-old ad libitum-fed and 26-month-old calorierestricted rats and of the livers (3) from 18- and 28-monthold ad libitum-fed and 28-month-old calorie-restricted rats were used. We found an age-related increase in TFAM amount in the frontal cortex, not affected by CR, versus an age-related decrease in the soleus and liver, fully prevented by CR. The semi-quantitative analysis of in vivo binding of TFAM to specific mtDNA regions, by mtDNA immunoprecipitation assay and following PCR, showed a marked age-dependent decrease in TFAM-binding activity in the frontal cortex, partially prevented by CR. An age-related increase in TFAM-binding to mtDNA, fully prevented by $\mathrm{CR}$, was found in the soleus and liver. MtDNA content presented a common age-related decrease, completely prevented by $\mathrm{CR}$ in the soleus and liver, but not in the frontal cortex. The common age-related loss of mtDNA might be explained by two different tissue-specific mechanisms. The age-related decrease in TFAM-binding in the frontal cortex might imply decreased mtDNA replication and/or increased mtDNA damage, not counteracted by the usual repair mechanisms. On the contrary, the age-related increased TFAM-binding at both origins of replication in the soleus and liver might explain the mtDNA loss through a hindered mtDNA replication. The modulation of TFAM expression, TFAM-binding to mtDNA and mtDNA content with aging and $\mathrm{CR}$ showed a trend shared by the skeletal muscle and liver, but not by the frontal cortex counterpart. A fine modulation of TFAM-binding to mtDNA may contribute to 


\title{
CIR-Myo News: Abstracts of the 2015 Spring Padua Muscle Days
}

\author{
Terme Euganee Padua (Italy), March 12 - 14, 2015
}

\section{Abstracts}

several tissue-specific changes involved in the age-related mitochondrial dysfunction as well as in the preventive effects of CR. This could have relevant consequences for future applications of CR or other nutritional regimens to delay the onset of pathologies, such as sarcopenia and neurodegeneration, related to the age-dependent mitochondrial dysfunction.

1. Picca A., Fracasso F., Pesce V., Cantatore P., Joseph A.M., Leeuwenburgh C., Gadaleta M.N., Lezza A.M.S. Age- and calorie restriction-related changes in rat brain mitochondrial DNA and TFAM binding, Age 2013;35:1607-20.

2. Picca A, Pesce V, Fracasso F, et al. A comparison among the tissue-specific effects of aging and calorie restriction on TFAM amount and TFAM-binding activity to mtDNA in rat. Biochim Biophys Acta 2014;1840:2184-91.

3. Picca A, Pesce V, Fracasso F, et al. Aging and calorie restriction oppositely affect mitochondrial biogenesis through TFAM binding at both origins of mitochondrial DNA replication in rat liver. PLoS One 2013:8 e74644, http://dx. doi.org/10.1371/journal.pone 0074644.

$$
* * * * *
$$

\section{Ageing causes severe ultra-structural modification of calcium release units and of mitochondria in cardiomyocytes}

Laura D’Onofrio*, Alessia Di Fonso*, Feliciano Protasi, Simona Boncompagni

* Equally contributing

CeSI - Center for Research on Ageing \& DNICS - Dept. Neuroscience, Imaging, and Clinical Sciences; University G. d'Annunzio of Chieti, Italy

E-mail: s.boncompagni@unich.it

Ageing is associated to a dramatic increase in the incidence of heart failure, even if the existence of a real age-related cardiomyopathy remains controversial. ${ }^{1-3}$ In this study we performed a morphological study of cardiac cells in hearts from adult and old mice ( 4 months and $\geq 24$ months of age, respectively) using confocal and electron microscopy. Our results indicates that the cross sectional areas (CSA) of cardiomyocytes is on the average increased in old hearts (adults: : $189 \pm 93 \mu^{2}$; aged: $282 \pm 155 \mu^{2}$ ), with the greater variability in size indicating also the presence of several atrophic cells. The increased average CSA may be the result of an increased presence of amorphic (and apparently empty) cytoplasmic space between myofibrils (adults: $2.2 \pm$ 0.3; aged: $9.7 \pm 0.6$ ). As effective contraction and relaxation of cardiomyocytes also depends on $\mathrm{Ca}^{2+}$ supply to myofibrils, handled by calcium release units (CRUs) and sarcoplasmic reticulum (SR) and on efficient ATP production (provided by mitochondria), we have also performed a qualitative and morphometric analysis of these intracellular organelles. The analysis of CRUs indicates that SR/transverse-tubules (TT) couplons becomes shorter with age and that the number of CRUs $/ 50 \mu \mathrm{m}^{2}$ is decreased of about $24 \%$ (adults: $5.1 \pm 3.3$; aged: $3.9 \pm 2.6$ ). Also mitochondria present structural modifications, with a significant increase in the percentage of organelles presenting severe alterations (3.5\% vs. $16.5 \%$, respectively in adult vs. aged). Importantly, both CRUs and mitochondria undergo a spatial re-organization with respect to sarcomeres/myofibrils: CRUs are may be miss-oriented (longitudinal) or miss-placed (found at the A band instead of being correctly placed in proximity of Z-lines), while mitochondria are often grouped in an abnormal fashion. These age-related ultra-structural changes may underlie an inefficient supply of $\mathrm{Ca}^{2+}$ and ATP to contractile elements, providing a possible structural explanation for heart dysfunction.

1. Boyle AJ, Shih H, Hwang J, et al. Cardiomyopathy of aging in the mammalian heart is characterized by myocardial hypertrophy, fibrosis and a predisposition towards cardiomyocyte apoptosis and autophagy. ExpGerontol 2011;46:549-59.

2. Wu H-D, Xu M, Li R-C,et al. Ultrastructural remodelling of $\mathrm{Ca} 2+$ signalling apparatus in failing heart cells. Cardiovasc Res 2012;95:430-8.

$$
* * * * *
$$

\section{Evolution of gravity detection and muscle tone}

\section{Hannes Petersen}

Department. of Anatomy, University of Iceland and dept. of Otorhinolaryngology, Landspitali University Hospital, Reykjavik, Iceland

E-mail: "Hannes Petersen" <hpet@hi.is>

Detection of the pull of earth's gravity (graviception) is essential to life and shared by all living things on earth, both plants and animals. Simple creatures as jellyfish through invertebrates have a simple form of graviception where biological mass (otoliths) act upon hair cells to detect the pull of gravity. With it's complex shape, sculpted in the petrosal part of the temporal bone, the mammalian inner ear is responsible of a dual function i.e. hearing and balance. The functional nominator is force transduction i.e. changing mechanical forces into electrical signals, called action potentials. ${ }^{1}$ The basis behind this force transduction property is two folded, i.e. inner ear morphological construction and physiological qualities. The later is based on potassium rich endolymph responsible for the endocochlear potential, hair cells depolarizing properties and recycling of potassium in the organ of Corti and nerve conduction of hair cell signal for central processing. The morphology of the inner ears are of highest interest, i.e. paired (right/left) construction with almost $100 \%$ symmetry not only regarding the size, but importantly regarding orientation of the semicircular canals, configuration of utricular and saccular maculae and coiling of the cochlea. This morphology was evolved in terrestrial environment in response to forces associated with sound conduction in air, gravity and fast head and body movements. In fish that live in aquatic environment, graviception is made possible through otolith that act upon hair cells within the utriculus and sacculus, i.e. big calcium crystals forms that allow the fish to detect the pull of earth's gravity, extremely reduced in the aquatic environment. During sea-to-land evolution the terrestrial environment forced upon the living creature much stronger gravity forces, that resulted in splitting of the otolith into otoconia, small calcium crystals embedded in gelatinous membrane in utriculus and sacculus of terrestrial vertebrates inner ears. Terrestrial environments allow also much quicker and more agile movements that calls 


\title{
CIR-Myo News: Abstracts of the 2015 Spring Padua Muscle Days
}

\author{
Terme Euganee Padua (Italy), March 12 - 14, 2015
}

\section{Abstracts}

upon more sophisticated semicircular canals which sets ground for vestibulo-ocular reflex essential for eye focusing. ${ }^{2}$ Muscle tone, the force with which a muscle resists being lengthened, depends on three fundamental issues. The intrinsic elasticity or stiffness of the muscles, the stretch reflex feedback loop and "higher" neural contribution. The fundamental of this higher neural contribution are signals from the inner ear i.e. the graviceptal information relayed through vestibulo-spinal pathways described partly by Sherrington some 100 years ago. ${ }^{3}$ Whales are mammals that have adapted holaquatic life style, where similar terrestrial mechanical forces are severely reduced and due to that the inner ear has changed dramatically again. The driving forces of those evolutional changes are probably due to seasickness.

1. Forge A and Wright $\mathrm{T}$. The molecular architecture of the inner ear. Br Med Bull 2002; 63: 5-24.

2. Beisel KW, Wang-Lundberg Y, Makland A, Fritzsch B. Development and evolution of the vestibular sensory apparatus of the mammalian ear. J Vest Res. 2005;15:225-41

3. Sherrington CS. 1906. Integrative Actions of the Nervous System. New Haven, CT: Yale Univ. Press.

$$
* * * * *
$$

\section{Postural Control of Elderly}

\section{Hannes Petersen}

Department of Anatomy, University of Iceland and dept. of Otorhinolaryngology, Landspitali University Hospital, Reykjavik, Iceland.

E-mail: "Hannes Petersen" <hpet@hi.is>

Dizziness, vertigo, impairment of balance and fear of falling are common complaints in elderly. The underlying processes are not fully understood but a significant number of symptoms have been linked to vestibular pathology, and inadequate postural control. Dizziness ranks among the most common complaints in medicine, affecting in some forms approximately $40 \%$ of general population during the course of life. ${ }^{1}$ In a Swedish cohort study the daily occurrence of balance problems was present in $33 \%$ among elderly at age 70 years and increased to $50 \%$ for elderly at age of 80 years or more. ${ }^{2}$ In literature the terms dizziness and vertigo have been used to identify the site of pathology, i.e. that dizziness would originate from non-vestibular sites and vertigo would be specific to the vestibular part of the inner ear. Finally the balance symptoms have been addressed less specifically to conflicts in postural control. The AGES Reykjavík study is based on the Reykjavik study, which started in 1967 and comprises health information of more than 20.000 individuals who at present are older than 69 years. The study, started in the year 2001 and is ongoing. The study includes several aspects of human aging and a total sample of more than 5000 subjects have been evaluated for balance and hearing functions, i.e. the ageing of CNS and its postural control have been thoroughly studied. ${ }^{3}$ Beside cognitive tests and MRI of the CNS, the focus is on following balance research: Questionnaire regarding brain, inner ear and balance problems. Motor functions test as timed up and go and 6 meter walk. Strength in the lower extremities.
Posturography (force) platform, where four main tests are performed: a) chair stand b) quiet stands with open and closed eyes c) target hunting d) step test. Hearing evaluation (PTA, impedens audiometry). Main findings are that the reaction time for both men and women decrease as well as the stabilization time in the chair test with increased age. Hearing thresholds in all frequencies tested decreases with increased age in both ears. All data available is now under thorough investigation schedule, which can be coupled with all other fields of data, harvested in the AGES Reykjavík study. The AGES Reykjavík study is one of the largest epidemiology studies in to ageing carried out.

1. von Brevern M, Radtke A, Lezius F, et al. Epidemiology of benign paroxysmal positional vertigo: a population based study. J Neurol Neurosurg Psychiatry 2007;78:663.

2. Jönsson R, Sixt E, Landahl S, Rosenhall U. Prevalence of dizziness and vertigo in an urban elderly population. J Vestib Res 2004;14:47-52

3. Rasku J, Pyykko I, Juhola M, et al. Evaluation of the postural stability of elderly persons using time domain signal analysis. J Vestib Res 2013;22:243-52.

$$
* * * * *
$$

Functional Magnetic Stimulation (FMS) and FES, useful synergic options with specific limitations

\section{Winfried Mayr, Matthias Krenn, Dietmar Rafolt}

Centre for Medical Physics and Biomedical Engineering, Medical University of Vienna, Vienna, Austria

E-mail: winfried.mayr@meduniwien.ac.at

FMS is a non-invasive technique to induce eddy currents in living tissue and activate action potentials in neuron structures. The main advantage in comparison to electrical stimulation via skin attached electrodes is the fact that pain sensors in the upper skin layers are not excited by FMS, as due to tissue impedance properties induced currents remain more or less below threshold. ${ }^{1,2}$ On the other hand, induction of effective electrical field strength requires power electronics capable of driving application coils with high impulse current in the range of $\mathrm{kA}$, which is an engineering challenge and rises problems of heat management and size and weight of equipment. Application of FMS has for long time been limited to delivering single and double stimuli to the human cortex for diagnostic assessments (transcranial magnetic stimulation), with bulky and expensive equipment. Just in the recent years, FMS devices enter the market that can deliver trains of stimuli with up to $100 \mathrm{~Hz}$ repetition rate and capable of inducing strong but comfortable muscular contractions. A first very useful application is offered for efficient and painless pelvic floor training for therapy of continence problems, with stimulator electronics and application coil integrated in a comfortable chair. Meanwhile also systems with stationary electronics module and mobile coil for manual positioning near target muscles are available, though at much higher costs than comparable electrical stimulators. Portable solutions are not realistically to be expected due to technical limitations. Even though we can expect, that FMS will become an important alternative treatment option where therapy stations can be provided in appropriate environment - like the mentioned pelvic chair centrally in retirement homes or outpatient clinics. Mobile 


\title{
CIR-Myo News: Abstracts of the 2015 Spring Padua Muscle Days
}

\author{
Terme Euganee Padua (Italy), March 12 - 14, 2015
}

\section{Abstracts}

solutions and affordable devices for home based training will in foreseeable future remain relying on FES technology.

1. Cretu M, Ciupa RV, Darabant L. Evaluation of Spinal Cord Response During Magnetic Stimulation of the Lumbar Area. Biomed Tech (Berl). 2013 Sep 7.

2. Neyroud D1, Temesi J, Millet GY, et al. Comparison of electrical nerve stimulation, electrical muscle stimulation and magnetic nerve stimulation to assess the neuromuscular function of the plantar flexor muscles. Eur J Appl Physiol. 2015 Feb 15. [Epub ahead of print].

$* * * * *$

\section{Recording of EMG signal within a prosthetic socket of a transfemoral amputee}

Jona Sigrun Sigurdardottir (1,2), Gudfinna Halldorsdottir (2), Stefan Pall Sigurthorsson (2), Kristleifur Kristinsson (2), Thordur Helgason $(1,3)$

(1) Institute of Biomedical and Neural Engineering, Health Technology Center, University of Reykjavik; (2) Landspitali - University Hospital Iceland, Reykjavik; (3) (2) Össur ehf. Reykjavik, Iceland (3) Landspitali - University Hospital Iceland, Reykjavik,, Iceland

E-mail: thordur@landspitali.is

EMG signals are a physiologically appropriate control source that can provide the user with direct control over a device and their exploration is an important step in the prosthetic development of an intuitive human-machine interface. ${ }^{1}$ Due to several technological hindrances, like high pressures and shear forces on the residual limb and thus upon surface electrodes within the socket, as well as traditional surface EMG drawbacks, ${ }^{1}$ no commercially available lower limb prosthetics yet utilise the surface EMG signal for control. This study explores a surface electrode measurement technique for attaining reliable EMG signals from the residual muscles of a transfemoral amputee, with the future prospect of controlling functions of lower limb prosthesis. Neuroline surface electrodes $\left(A m b u{ }^{\circledR}\right.$ Denmark) ${ }^{2}$ were used inside the socket, on the hamstring, quadriceps and adductor muscles, and passed proximally above the socket rim to the ground electrode on the subjects back. Nickel-plated brass triode electrodes(T340 Lifematters US) ${ }^{4}$ were placed outside of the socket, on the tensor fasciae latae (TFL) and gluteus medius (GM) muscles and were also used as reference electrodes. Placing of electrodes on the TFL and GM muscles followed the SENIAM recommendations. ${ }^{4}$ Same recommendations could not be applied for muscles whose distal end had been amputated but instead the optimal electrode position was determined by palpation and the area of the strongest signals was defined by iterative placement of the triode electrodes in the area of interest, taking in account the anticipated displacement by the liner and socket (usually proximal direction). An array of 3 to 4 electrode pairs were placed over the region of greatest activity and the EMG signals were measured after the amputee donned his socket to locate the best electrode position. The EMG signals were recorded and analyse using the wireless Kine System (Kine ehf. Reykjavík, Iceland). ${ }^{5}$ EMG signals were collected while the subject contracted individually the respective muscles, first prior to liner placement and again after liner and socket placement. The same signals were subsequently collected collectively during treadmill walking, stair decent and sittingto standing. The study shows that EMG signals can with this method be reliably identified and collected using surface electrodes underneath a silicone liner. The signals were more distinguished after liner and socket placement. The technique for placement of an array of electrodes was critical for success as it permits anticipation of the displacement by liner and socket system during weight bearing. This technique does not make the placement of electrodes practical for home use. Still we conclude that the results are promising regarding the prospects of using an array of surface EMG signals for the control of lower limb bionic prosthesis.

1. M. Ortiz-Catalan, R. Brånemark, B. Håkansson, and J. Delbeke, "On the viability of implantable electrodes for the natural control of artificial limbs: Review and discussion," Biomed. Eng. OnLine, vol. 11, no. 1, p. 33, Jun. 2012.

2. Ambu, "Ambu Neuroline 720," 2013. [Online]. Available:

http://www.ambu.com/corp/search/product/neuroline_72 0-prod307.aspx?PID=22206. [Accessed: 23-Feb-2015].

3. Lifematters, "EMG Electrodes, Triode Pads T3402M," , (n.d.). [Online]. Available: http://www.lifematters.com/emgpads-triode.asp.

[Accessed: 23-Feb-2015].

4. European Commission, Directorate General for Research, Commission of the European Communities, and Biomedical and Health Research Programme, SENIAM: European recommendations for surface electromyography. Netherlands: Roessingh Research and Development, 1999.

5. Kine.is, "KINE wireless surface electromyography (SEMG)" (n.d.). [Online]. Available: http://kine.is /Products/MMS/. [Accessed: 23-Feb-2015].

$$
* * * * *
$$

\section{Advanced processing of electromyography signals in measurements of trunk postural actions}

Nejc Sarabon $(1,2)$ and Andrej Panjan (2)

(1) University of Primorska, Andrej Marusic Institute, Department of Health Study, Koper, Slovenia; (2) S2P, Science to Practice, d.o.o., Laboratory for Motor Control and Motor Behaviour, Ljubljana, Slovenia

E-mail: nejc.sarabon@s2p.si

Low back pain is the most common health problem of musculo-skeletal system of today's population. It is a big social and economic problem in developed countries and in developing countries. People with low back pain have many changes in neuro-muscular functions of the human trunk. ${ }^{1-3}$ To assess postural control muscle activation during mechanical perturbation is measured with the use of electromyography (EMG). Processing of EMG signals is usually done manually and the expert must have good knowledge of the used methods. ${ }^{4,5}$ This makes it very slow, error prone and unsuitable for large set of EMG signals. Our aim in this study was to develop methods that will be suitable for automatic processing of EMG signal with no user input. Processing of EMG signals can be divided into three phases: 


\title{
CIR-Myo News: Abstracts of the 2015 Spring Padua Muscle Days
}

\author{
Terme Euganee Padua (Italy), March 12 - 14, 2015
}

\section{Abstracts}

noise removal, detection of a response onset and offset and assessment of an event (attribute calculation). In noise removal phase we must deal with several types of noises from which the electrocardiogram (ECG) artifact is very common in EMG signals of the trunk and it is very hard to remove. ${ }^{6}$ Several methods (high-pass filtering, gating, template subtraction, empirical mode decomposition with independent component analysis, adaptive wavelet transform) have been proposed in the literature to remove ECG artifact, however, none of them turned out to be completely successful. We have applied, tested and proposed a new method for ECG artifact removal that is based on dynamic time warping method and it first identifies ECG artifacts in the EMG signal and then adaptively removes the artifacts with subtraction. For onset and offset detection a variety of different approaches has been used (methods based on threshold, optimal estimator, approximated generalized likelihood-ratio detector (AGLR), integrated profile, sample entropy and some others). We developed a new method for this task that is based on AGLR and have more advanced false event detector which helps eliminates false detections. There has been several attributes for an event assessment used in the literature: attributes in time domain, attributes in frequency domain and attributes in time-frequency domain. We add several attributes known from needle EMG and sound processing domain and identified subsets that are the best for complete EMG event assessment. Our proposed method for ECG artifact removal outperformed other methods and proved to be more reliable in ECG detection. AGLR with advanced false detector showed better results compared to previously used methods, especially in false detection prevention. A few subsets for complete EMG event assessment were identified as suitable for this task. Newly introduced attributes from needle EMG and sound processing domain improved the reliability significantly. Newly proposed methods makes EMG processing more reliable, much faster and are suitable to process large datasets of EMG signals from human trunk.

1. Ortiz-Catalan M, Brånemark, B. Håkansson, and J. Delbeke, "On the viability of implantable electrodes for the natural control of artificial limbs: Review and discussion,” Biomed. Eng. OnLine, vol. 11, no. 1, p. 33, Jun. 2012.

2. Ambu, “Ambu Neuroline 720," 2013. [Online]. Available:http://www.ambu.com/corp/search/product/ neuroline_720-prod307.aspx?PID=22206. [Accessed: 23Feb2015].

3. Lifematters, "EMG Electrodes, Triode Pads T3402M,", (n.d.). [Online]. Available: http://www.lifematters.com/ emgpads-triode.asp. [Accessed: 23-Feb-2015].

4. European Commission, Directorate General for Research, Commission of the European Communities, and Biomedical and Health Research Programme, SENIAM: European recommendations for surface electromyography. Netherlands: Roessingh Research and Development, 1999.

5. Kine.is, "KINE wireless surface electromyography (SEMG)" (n.d.). [Online]. Available: http://kine.is/ Products/MMS/. [Accessed: 23-Feb-2015].

\section{Patient Data Concerning EMG-Based On-Demand Predictive Control of DBS for Parkinson Patients and Significance}

Daniel Graupe (1,2), Nivedita Khobragade (1), Daniel Tuninetti (1), Konstantin V Slavin (3), Leonard Verhagen Metman (4), Ahmed Rabie (3)

(1) Department of Electrical and Computer Engineering, University of Illinois, Chicago; (2) Department of Neurology and Rehabilitation Medicine, University of Illinois, Chicago; (3) Department of Neurosurgery, University of Illinois, Chicago; 4. Department of Neurological Sciences, Rush University Medical Center, Chicago, IL. USA

E-mail: graupe@uic.edu

The paper is concerned with closed-loop On-Demand control of Deep-Brain Stimulation (DBS) for Parkinson's disease (PD) patients and it efficacy. On-Demand control implies that DBS is applied in pulse trains of finite pre-computed durations only when necessary, rather than being applied continuously. The paper thus investigates the efficacy of OnDemand DBS control, in terms of maximizing the ratio of durations where no tremor occurs while stimulation is OFF, relative to the duration of stimulation that precedes the period of no-stimulation. It also investigates the efficiency of a neural-network-based algorithm to predict onset of tremor in order that On-Demand control of DBS will avoid any tremor from occurring via efficient prediction of onset of tremor before rather than after it occurs. Data were obtained from 9 PD patients, tested at the University of Illinois Hospital in Chicago, IL, all of whom had been implanted with DBS in at the University of Illinois Hospital, Chicago or at Rush University Medical Center, Chicago over the past 15 years. Non-invasive surface EMG (sEMG) data from patients' limbs were used to predict onset of tremor stochastic signal processing algorithms, including entropy analysis, were used to process the raw sEMG signals. During testing, DBS stimuli of standard and FDA-approved protocols were applied in packets of pulse-trains of varying durations. We show that there exists, for each patient, an average duration of Stimulation that maximizes the ratio of duration of NoTremor-No-Stimulation over duration of Stimulation (denoted as R). In 3 out of 9 patients tested above, the ratio $\mathrm{R}$ (as above) is between 2 and 10.3, in the vast majority of trials performed in these patients ( 25 of 30 individual trials), when a stimulation duration that maximizes $\mathrm{R}$ was used. In conclusion, we show that: a) On-Demand control of DBS using non-invasive sEMG sensors can reduce total stimulation time by $55 \%$ to over $90 \%$ (namely $[R+1] / R$ ), depending on the patient involved, in a significant number of PD patients with tremor, when adequately selected durations of stimulation pulse-trains are employed; b) An optimal duration of stimulation pulse-trains as in (a) exists and is computable; c.) Prediction of onset of tremor from noninvasive sEMG signals is possible, to allow applying fixed duration DBS pulse-trains before actual tremor starts. Our past work [Basu et al., J Neural Engineering, vol. 10, No. 3, 2013] on predicting onset of tremor following a DBS pulsetrain via sEMG yields the average duration (Tp) from end of stimulation to predicted tremor to be more than 0.8To where To is the duration from end of stimulation to onset of actual tremor. Hence, an on-demand predictive controller, using the 


\title{
CIR-Myo News: Abstracts of the 2015 Spring Padua Muscle Days
}

\author{
Terme Euganee Padua (Italy), March 12 - 14, 2015
}

\section{Abstracts}

latter or similar prediction algorithm, makes a non-invasive sEMG-based ON-DEMAND control of DBS feasible and effective for at least a sub-population of PD patients who have tremor. It requires no change in present (uncontrolled) DBS implantation procedures or in DBS pulse-generation protocols already approved by the regulatory authorities; d) Prediction can be made in sufficient time ahead of the onset of tremor, while still keeping the tremor-free time without stimulation reasonably long compared to the duration of stimulation being on; e) It is reasonable to use tremor as a proxy for all Parkinsonian symptoms (i.e. rigidity, bradykinesia, etc.), as tremor is the first symptom to reappear after DBS is switched off and the first symptom to resolve after DBS is turned back on. ${ }^{1}$

1. Temperli P, Ghika J, Villemure JG, et al. How do parkinsonian signs return after discontinuation of subthalamic DBS? Neurology 2003;14;60:78-81.

$* * * * *$

\section{Histone deacetylase 4 is protective in ALS and modulates} the response to oxidative stress

Eva Pigna (1), Rosa Mancinelli (2), Dario Coletti, (3), Sergio Adamo (1), Viviana Moresi (1)

(1) Sapienza University of Rome, Department of Anatomical, Histological, Forensic \& Orthopaedic Sciences, Histology \& Medical Embryology Section, Rome, Italy; (2) Department of Neuroscience, Imaging and Clinical Sciences, University G. d'Annunzio Chieti-Pescara, Chieti, Italy; (3) Pierre and Marie Curie University - Paris 6 Vieillissement, stress, inflammation (UR 4), Paris, France

E-mail: "Viviana Moresi" <viviana.moresi@uniroma1.it>

Amyotrophic lateral sclerosis (ALS) is a progressive neurodegenerative disease characterized by motor neuron degeneration, muscle atrophy and weakness, eventually leading to muscle paralysis and death. Several factors account for the development of ALS, including accumulation of oxidative stress in skeletal muscle1. A positive correlation between the expression of the histone deacetylase 4 (HDAC4) and the progression of the disease has been recently reported in ALS patients, suggesting the use of HDAC4 inhibitors as a promising therapeutic approach for the treatment of this neurodegenerative disease2. HDAC4 in skeletal muscle plays a crucial role in the regulation of muscle mass and reinnervation following denervation3. However, the molecular pathways controlled by HDAC4 in ALS onset or progression, as well as in response to oxidative stress in skeletal muscle are not delineated yet. We investigated the role of HDAC4 in ALS by deleting HDAC4 in skeletal muscle of SOD1G93A mice, a mouse model of ALS. Lack of HDAC4 in skeletal muscle anticipated body weight loss and induced more pronounced muscle atrophy in late stage SOD1G93A HDAC4 mKO mice, compared with age-matched SOD1G93A mice, indicating a protective role of HDAC4 in ALS. To study the molecular mechanisms underlying HDAC4 function in response to a chronic denervation, such as in ALS, we cut the sciatic nerve of one limb of HDAC4 mKO mice and analyzed muscles over time. HDAC4 mKO mice did not undergo muscle atrophy for two weeks following denervation, but muscles degenerated at later time points. Moreover, contralateral innervated muscle of HDAC4 mKO mice presented ultrastructural defects in myofiber organization and higher levels of ROS, while alteration of sarcomeric architecture and the molecular responses to oxidative stress were blunted following denervation. From our results, we conclude that HDAC4 protects skeletal muscle in ALS and is important to maintain muscle integrity and oxidative stress response following denervation. Further studies are necessary to delineate the role of HDAC4 in skeletal muscle integrity and in response to chronic denervation.

1. Musaro A. State of the art and the dark side of amyotrophic lateral sclerosis. World J Biol Chem 2010;1:62-8.

2. Bruneteau G. Muscle histone deacetylase 4 upregulation in amyotrophic lateral sclerosis: potential role in reinnervation ability and disease progression. Brain 2013;136(Pt 8):2359-68.

3. Moresi V Myogenin and class II HDACs control neurogenic muscle atrophy by inducing E3 ubiquitin ligases. Cell 2010;143:35-45. doi: 10.1016/j.cell. 2010.09.004.

$$
* * * * *
$$

\section{Leg Muscle Electrical Stimulation in Amytrophic Lateral} Sclerosis

Alfonc Baba (1), Antonio Merico (1), Andrea Marcante (1,2), Kiper Paweł (1), Rossi Simonetta (1), Masiero Stefano (2), Ugo Carraro (1), Francesco Piccione (1)

(1) Department of Neurorehabilitation, IRCCS Foundation Hospital San Camillo, Venice, Italy; (2) Department of Neuroscience, University of Padua, Italy

E-mail: Alfonc.baba @ ospedalesancamillo.net

Amyotrophic Lateral Sclerosis (ALS) is a major neurodegenerative disorder, which involves the degeneration of upper and lower motor neurons, muscle weakness, atrophy, fasciculations and paralysis. The deambulation is quantitatively and qualitatively reduced. However, pervious evidence in related field suggests usefulness of conventional rehabilitation for the ALS patients. Furthermore, there is no evidence regarding the specific treatment modality. Functional Electrical Stimulation (FES) has been assessed for neurorehabilitation treatment (Spinal Cord Injuries, Stroke, Multiple Sclerosis), but there are shortage scientific reports for the ALS. Relying on neurophysiological mechanisms, we hypothesized that FES could be a beneficial muscle's treatment in the patients affected by ALS. Therefore the aim of the present study is to investigate the clinical and functional effects of the electrical muscle stimulation in ALS patients. Matherials and Methods: Two patients, admitted to the Department of Neurorehabilitation of the Care \& Research Istitute San Camillo in Venice, with confirmed primary ALS were treated according to the experimental Cycling-FES clinical protocol. The protocol was an additional 1 hour a day treatment to the conventional neuromotor rehabilitation. The lower limbs training was performed by the use of Cycling-FES (Hasomed RehaStim2 MOTOmed® viva2). The treatment consisted of 15 sessions, half an hour a day, 5 days per week, for 3 weeks. The therapeutic session was divided as follows: 5 minutes of 


\section{CIR-Myo News: Abstracts of the 2015 Spring Padua Muscle Days}

Terme Euganee Padua (Italy), March 12 - 14, 2015

\section{Abstracts}

warm-up (pedal without FES), 20 minutes pedal + FES and 5 minutes of cool-down (pedal without FES). The following muscles (bilateral) were considered for the FES: flexors and extensors of the knee, dorsal and plantar flexor of the ankle. The surface adhesive electrodes were placed according to the operation manual of Rehastim 2 (i.e. $9 X 4 \mathrm{~cm}$ for the proximal muscles and $4 \mathrm{X} 4 \mathrm{~cm}$ for the distal muscles). The parameters of electrical stimulation were as follows: biphasic rectangular waveform, frequency range $40-45 \mathrm{~Hz}$, pulse duration within $150-200 \mu \mathrm{sec}$, and the muscles contraction was obtained through intensity within $30-50 \mathrm{~mA}$, as well. The synchronized cycling was provided by a motorized cycle ergometer (20-30 Revolutions per Minute). Functional tests and clinical scales [i.e. 10 Meters \& 6 Minutes Walking Test; Medical Research Council (MRC) Scale for Muscle Strength; Timed Up \& Go; Modified Ashworth Scale] were performed, before and after treatment, in order to assess the effects of therapy. All the same, was monitored the spasticity, through an instrumental examitation (H-reflex test). Results: Both subjects improved their motor performance according to the clinical and functional tests. Furthermore, the modified Ashworth Scale and H-reflex test did not show increased spasticity of lower limb muscles. There were no reports of adverse events during the assessment and treatment sessions, as well. Discussion and Conclusions: To our knowledge this is the first study which described the application of CyclingFES for the ALS treatment. Obtained results are encouraging, but, due to limited subjects, we cannot draw any conclusion, therefore we are keeping on working, because further research is needed.

U.C. thanks the Interdepartmental Research Center of Myology at the Department of Biomedical Sciences, University of Padova, Italy for collaboration and hospitality and the Ludwig Boltzmann Institute of Electrical Stimulation and Physical Rehabilitation of Vienna at the Department of Physical Medicine, Wilhelminenspital, Vienna, Austria for support and collaboration.

1. Young CA, Gibbons C, Pagnini F, Friedee T. Treatment for fatigue in amyotrophic lateral sclerosis/motor neuron disease (ALS/MND), Review, Cochrane Database of Systematic Reviews 2014 Published Online: 2 MAR 2014. doi: 10.1002/14651858.CD011005

2. Dal Bello-Haas V, Florence JM, Krivickas LS. Therapeutic exercise for people with amyotrophic lateral sclerosis or motor neuron disease (Review) Cochrane Database Syst Rev 2008;(2):CD005229. doi: 10.1002/ 14651858.CD005229.pub2.

3. Gordon PH. Amyotrophic Lateral Sclerosis: An update for 2013 Clinical Features, Pathophysiology, Management and Therapeutic Trials, 2013, Aging Dis 2013;4: 295-310. doi: 10.14336/AD.2013.0400295.

4. A Chen, J Montes, H Mitsumoto. The role of exercise in Amyotrophic Lateral Sclerosis. Phys Med Rehabil Clin N Am 2008; 19: 545-557.

$$
* * * * *
$$

Functional electrical stimulation: a possible strategy to improve muscle function in Central Core Disease?

Pierpaolo Iodice(1), Simona Boncompagni(1), Stefan Loefler (2), Christian Hofer (2), Lucia Galli(3), Antonio Di Muzio (4), Vincenzo Sorrentino(3), Helmut Kern (2,5), Feliciano Protasi (1)

(1) 1CeSI - Center for Research on Ageing \& DNICS - Dept. of Neuroscience, Imaging, and Clinical Sciences; University G. d'Annunzio, I-66100 Chieti Italy; (2) Ludwig Boltzmann Institute of Electrical Stimulation and Physical Rehabilitation, A-1160 Vienna Austria; (3) Department of Molecular and Developmental Medicine \& University of Siena and Azienda Ospedaliera Universitaria Senese, I-53100 Siena Italy; (4) Center for Neuromuscular Diseases \& Ospedale Clinicizzato SS Annunziata, I-66100 Chieti Italy; (5) Institute of Physical Medicine and Rehabilitation, Wilhelminenspital, A-1160 Vienna Austria.

E-mail: f.protasi@unich.it

Central Core Disease (CCD; OMIM\# 117000), one of the most common human congenital myopathies, is characterized by hypotonia and proximal muscle weakness with slow (or non progressive) clinical course. ${ }^{1}$ Diagnosis of CCD is confirmed by histological examination of muscle biopsies showing amorphous central areas or cores (typically found in type I muscle fibers), lacking glycolytic/oxidative enzymes and mitochondria. Usually, orthopedic complications limit the ability of CCD adult patient to perform physical exercise. Most CCD families have been associated with $\mathrm{C}$-terminal mutations in the gene encoding for the $\mathrm{Ca}^{2+}$ release channel of skeletal fibers, i.e. ryanodine receptor type-1 (RYR1). We have recently successfully used functional electrical stimulation (FES) to rescue muscle mass and force in spinal cord injury patients and in elderly subjects, ${ }^{2,3}$ but the use of FES has never been considered to improve muscle function in CCD patients. The purpose of this study is to test the efficacy of FES in counteracting muscle loss and improve function in the lower extremities of a 55-year-old female patient affected by CCD. The patient presented, since the adolescence, fatigue and progressive proximal limb weakness with hyperCKaemia. Her family history was inconsistent for neuromuscular diseases. At 44 years of age she was diagnosed with CCD as a muscle biopsy in the left brachial biceps revealed the presence of "central cores" in most type1 muscle fibers. Methods. a) Genetic Screening. PCR primers for all RYR1 exons were designed with the Primer-3 software (http://frodo.wi.mit.edu/cgibin/primer3/primer3_ www.cgi). Mutation analysis of the RYR1 gene has been performed as previously described $;{ }^{4}$ b) FES protocols. Before beginning of FES training protocols, the patient performed stabilometry test (1), maximal isometric voluntary force (MVF) of leg extensor muscle test by dynamometer (2) and a complete set of functional tests to assess mobility and function in activities of daily living (ADL). These tests included: time up and go test (3, TUGT); 10m-walking test with habitual and fastest walking speed (4) and short physical performance battery (5, SPPB). In the first phase of FES training (5 months) from Time-0 to Time- 1 (T0 to T1), upper leg muscles of the patient have been stimulated by FES $(60 \mathrm{~Hz}), 3$ sessions per week, $3 \times 10 \mathrm{~min}$ of treatment for each muscular group. c) Electron microscopy (EM) analysis. A 


\title{
CIR-Myo News: Abstracts of the 2015 Spring Padua Muscle Days
}

\author{
Terme Euganee Padua (Italy), March 12 - 14, 2015
}

\section{Abstracts}

new muscle biopsy was collected from the right vastus lateralis using a semi-automatic needle (Precisa 13 Gauge; Hospital Service, Rome, Italy) to perform EM analysis. Neurological examination revealed a hyperlordotic posture, bilateral pes cavus, limb-girdle hypostenia (MRC score: 3-4 in the upper and 2-3 in the lower) with waddling gait and difficulty into climbing stairs, steppage. The study is still in progress: EM structural analysis has been only performed at T0, whereas most functional assessments have been so far only performed at $\mathrm{T} 1=5$ months). Analysis of the RYR 1 gene identified a missense mutation $(c .7354 \mathrm{C}>\mathrm{T})$ resulting in the substitution of arginine in position 2452 with a tryptophan (p.R2452W). In addition, a duplication of 4 amino acid residues (Thr, Ala, Ala, Thr: p.Thr4285Thr4288dup) was also observed. At the EM ultrastructural analysis, a high percentage of muscle fibers analyzed (80\%) revealed the presence of large regions of hyper-contraction devoid of intra-myofibrillar organelles, i.e. calcium release unites (CRUs) and mitochondria. The outcome of the functional tests at $\mathrm{T} 1$ was only partially encouraging: whereas improvement in the Stabilometric and MVF tests were encouraging (indication of an improvement of force induced by FES), results in the battery of ADL tests were unfortunately negative. The reason for this partially negative outcome may reside in low back-pain lamented in the last months by the patient, a problem that has temporally caused a significant reduction in her daily walking activities. In conclusion, the next immediate goal is, obviously, to determine the reason causing low back-pain and reduced mobility of the patient (by magnetic resonance imaging). The study will then proceed in the next months to a) stabilize the lower back of patients with the goal of improving her mobility and independence and b) proceed with the next step in FES training (at increasing loads) with the goal of improving ADL functional tests. A new muscle biopsy for EM will be performed only if (and when) functional performance will be significantly improved.

1. Jungbluth H.. Central core disease. Orphanet J Rare Dis 2007:2:25.

2. Kern H, Carraro U, Adami N,. Home-based functional electrical stimulation rescues permanently denervated muscles in paraplegic patients with complete lower motor neuron lesion. Neurorehabil Neural Repair 2010;24:709.

3. Kern H, Barberi L, Löfler S, et al. Electrical stimulation counteracts muscle decline in seniors. Front Aging Neurosci 2014;6:189.

4. Galli L, Orrico A, Lorenzini S, et al. Frequency and localization of mutations in the 106 exons of the RYR1 gene in 50 individuals with malignant hyperthermia. Hum Mutat 2006;27:830.

$$
* * * * *
$$

\section{SAVe-ALS Project: An Update}

Ugo Carraro (1), Sandra Zampieri (2,3), Francesco Piccione (1), Andrea Marcante (1,4), Stefano Masiero (4), Vincenzo Vindigni (5), Marco Patruno (6), Michela Pozzobon (7), Helmut Kern (3,8), Janez Rozman (9), Janez Zidar (10), Amber Pond (11), Jonathan Jarvis (12), Winfried Mayr (13)

(1) IRCCS Fondazione Ospedale San Camillo, Venezia, Italy; (2) Interdepartmental Research Center of Myology
(CIR-Myo), Department of Biomedical Science, University of Padova, Italy; (3) Ludwig Boltzmann Institute of Electrical Stimulation and Physical Rehabilitation, Vienna, Austria; (4) Department of Neuroscience, Rehabilitation Unit, Padua University Hospital, Padova, Italy; (5) CIR-Myo at the Department of Neuroscience, Plastic Surgery Unit, University of Padova, Italy; (6) CIR-Myo at the Department of Comparative Biomedicine and Food Science, University of Padova, Italy; (7) Fondazione Istituto di Ricerca Pediatrica Città della Speranza, Padova, Italy; (8) Department of Physical Medicine, Wilhelminenspital, Vienna, Austria; (9) Center for Implantable Technology and Sensors, ITIS d. o. o. Ljubljana, and Institute of Pathophysiology, Medical Faculty, University of Ljubljana, Slovenia; (10) Institute of Clinical Neurophysiology, University Medical Centre Ljubljana, Slovenia; (11) Anatomy Department, Southern Illinois University School of Medicine, Carbondale, Illinois, USA; (12) Liverpool John Moores University, UK;(13) Centre for Medical Physics and Biomedical Engineering, Medical University of Vienna, Austria

E-mail: ugo.carraro@unipd.it@unipd.it

Amyolateral Sclerosis (ALS) is a neurodegenerative disease, attacking motor neurons. The degeneration of the motor neurons causes muscle paralysis and death will eventually result, often from a ventilation crisis. Only a small percentage of ALS cases are known to be genetic. The causes of sporadic ALS cases (those without a known genetic origin) are not understood. There is no known cure for ALS and only palliative therapies are available, at least for the predictable future. ${ }^{1}$ However, we believe that respiratory muscle function may be extended, thus postponing the need for pumping air which further damages the diaphragm muscle. We must first show in animal models that some additional muscle contractile function can be achieved by combining proven approaches to maintain/recover contractility of "denervated" muscle fibers of the diaphragm. ${ }^{2-4}$ The aim of the Project Save-ALS is to experimentally test a number of procedures on animals experiencing unilateral sciatectomy of leg muscles and unilateral section of the phrenic nerve. Working with mammals, first with rodents (rats and mice, the last both wild type and $\left.\mathrm{SOD}^{-/}\right),{ }^{6-8}$ and then with larger mammals (e.g., rabbits, sheep, and pigs) we will test the effects of the following: 1) new molecules, 2) new improved methods of production and injections of gliogenic and myogenic stem cells derived from adipose tissue, and 3) effects of physical stimuli (i.e., direct electrical stimulation of denervated muscle fibers in the leg muscles and the diaphragm, using flexible ring multi-wire electrodes). The development of new protocols and stimulation devices (e.g., electrodes and implantable mini-stimulators ) for denervated muscle will complement the effects of existing commercial electrostimulators used for ventilation support to delay the necessity for use of supported ventilation by pneumatic devices. This is important because controlled mechanical ventilation is known to exacerbate this progressive disease by unloading the diaphragm, thus decreasing the diaphragmatic force generating capacity and potentially causing ventilatorinduced diaphragmatic dysfunction (VIDD Syndrome) ${ }^{9-11}$ In short, we would like to test if the accumulating evidence that brief ES, a useful method to improve functional recovery for 


\section{CIR-Myo News: Abstracts of the 2015 Spring Padua Muscle Days}

Terme Euganee Padua (Italy), March 12 - 14, 2015

\section{Abstracts}

delayed repair of peripheral nerve lesions, may be extended to ALS. ${ }^{12-15}$ It is important to note that devices already were approved for ALS-treatment and are reported to prolong life expectancy based on a large study (http://www.synapsebiomedical.com/). Nevertheless, the reported devices work by activating the remaining innervated muscle fibers and not the denervated ones (http://www.synapse biomedical.com/als/neurx-als.shtml). To test if denervated fibers could be recovered, we will continue preliminary experiments in oldest-old rats $(>30$ months of age) which show that, even in these trial animals, short-term phrenicotomy does not significantly change the contractile characteristics of the diaphragm, when tested in vitro. If activation of muscle by ES can improve the setting, differentiation and survival of myogenic and gliogenic stem cells administered to the experimental animals, then it will be an achievable goal to support ventilation in ALS subjects. Thus, our goal will be able to design and implement a multifaceted strategy based on activity-driven enhancement of the effects of molecular, cellular and physical approaches.

U.C. thanks the Interdepartmental Research Center of Myology at the Department of Biomedical Sciences, University of Padova, Italy for collaboration and hospitality and the Ludwig Boltzmann Institute of Electrical Stimulation and Physical Rehabilitation of Vienna at the Department of Physical Medicine, Wilhelminenspital, Vienna, Austria for support and collaboration.

1. Wijesekera LC, Leigh NP. Amyotrophic lateral sclerosis. Orphanet J Rare Dis 2009; 4:3.

2. Kern H, Boncompagni S, Rossini K, et al. Long-term denervation in humans causes degeneration of both contractile and E-C coupling apparatus that can be reversed by functional electrical stimulation (FES). A role for myofiber regeneration? J Neuropath Exp Neurol 2004;63:919-31.

3. Boncompagni S, Kern H, Rossini K, et al. Structural differentiation of skeletal muscle fibers in the absence of innervation in humans. Proc Natl Acad Sci U S A 2007;104:19339-44.

4. Kern H, Carraro U, Adami N, et al. Home-based functional electrical stimulation rescues permanently denervated muscles in paraplegic patients with complete lower motor neuron lesion. Neurorehabil Neural Repair 2010; 24:709-21.

5. Dobrowolny G, Aucello M, Rizzuto E, et al. Skeletal muscle is a primary target of SOD1G93A-mediated toxicity. Cell Metab 2008;8:425-36.

6. Howland DS, Liu J, She Y, et al. Focal loss of the glutamate transporter EAAT2 in a transgenic rat model of SOD1 mutant-mediated amyotrophic lateral sclerosis (ALS). Proc Natl Acad Sci U S A 2002; 99:1604-9.

7. Kemp SW, Phua PD, Stanoulis KN, et al. Functional recovery following peripheral nerve injury in the transgenic Thy1-GFP rat. J Peripher Nerv Syst 2013 18:220-1.

8. .http://www.synapsebiomedical.com/fdaapproval/intende dUse-ALS.shtml

9. Goligher EC, Ferguson ND, Kavanagh BP. Ventilatorinduced diaphragm dysfunction: from mice (hopefully) to men. Anesthesiology 2012;117:463-4.
10. Bourke SC, Tomlinson M, Williams TL, et al., Effects of non-invasive ventilation on survival and quality of life in patients with amyotrophic lateral sclerosis: a randomized controlled trial. Lancet Neurol 2006;5:140-7.

11. Mosole S, Carraro U, Kern H, et al., Long-Term highlevel exercise promotes muscle reinnervation with age. 2014;73:284-94. doi: 10.1097/NEN.0000000000000032.

12. Mitchell WK, Williams J, Atherton P, et al. Sarcopenia, dynapenia, and the impact of advancing age on human skeletal muscle size and strength; a quantitative review. Front Physiol 2012; 3:260.

13. Gargiulo P, Reynisson PJ, Helgason B, et al., Muscle, tendons, and bone: structural changes during denervation and FES treatment. Neurol Res. 2011;33:750-8.

14. Pradat PF, Barani A, Wanschitz J, et al., Abnormalities of satellite cells function in amyotrophic lateral sclerosis. Amyotroph Lateral Scler 2011;12:264-71. doi: 10.3109/17482968.2011.566618. Epub 2011 Apr 8.

$$
* * * * *
$$

\section{P3. Tissue-specific effects on TFAM amount, mtDNA and TFAM-binding to mtDNA in aged and calorie-restricted rat}

Anna Picca (1), Vito Pesce (1), Flavio Fracasso (1), AnnaMaria Joseph (2), Christiaan Leeuwenburgh (2), Angela Maria Serena Lezza (1)

(1) Department of Biosciences, Biotechnologies and Biopharmaceutics, University of Bari, Italy; (2) Department of Aging and Geriatric Research, Institute on Aging, Division of Biology of Aging, University of Florida, Gainesville, FL, USA

E-mail: angelamariaserena.lezza@uniba.it

Mitochondrial Transcription Factor A (TFAM) is a histonelike protein for mitochondrial DNA (mtDNA), involved in multiple functions for this genome. Aging markedly affects mitochondrial biogenesis and functions in a tissue-specific manner and calorie restriction (CR) diet is, so far, the only intervention able to delay or prevent the onset of several agerelated alterations, also in mitochondria, in different organisms. TFAM amount, mtDNA content and TFAMbinding to mtDNA were analyzed in samples of frontal cortex and soleus skeletal muscle from 6- and 26-month-old ad libitum-fed and 26-month-old calorie-restricted rats and of liver from 18- and 28-month-old ad libitum-fed and 28month-old calorie-restricted rats. We found an age-related increase in TFAM amount in the frontal cortex, not affected by $\mathrm{CR}$, whereas an age-related decrease was present in the soleus and liver, fully prevented by CR. The semiquantitative analysis of in vivo binding of TFAM to specific mtDNA regions, by mtDNA immunoprecipitation assay and following PCR, showed a marked age-dependent decrease in TFAM-binding activity in the frontal cortex, partially prevented by CR. An age-related increase in TFAM-binding to mtDNA, fully prevented by CR, was found in the soleus and liver. A common age-related decrease in mtDNA content, completely prevented by $\mathrm{CR}$, was found in the soleus and liver, but not in the frontal cortex. The modulation of TFAM expression, TFAM-binding to mtDNA and mtDNA content with aging and CR showed a trend shared by the skeletal muscle and liver, but not by the frontal 


\title{
CIR-Myo News: Abstracts of the 2015 Spring Padua Muscle Days
}

\author{
Terme Euganee Padua (Italy), March 12 - 14, 2015
}

\section{Abstracts}

cortex counterpart. Considering the above mentioned findings, aging and $\mathrm{CR}$ appear to induce similar mitochondrial molecular mechanisms in the skeletal muscle and liver, different from those elicited in the frontal cortex.

$* * * * *$

\section{P4. FES training protocols for the functional recovery of permanently complete denervated human muscles}

\section{Löfler S (1), Mayr W (2), Moedlin M (3), Burggraf S (1),} Kern H $(1,3)$

(1) Ludwig Boltzmann Institute of Electrical Stimulation and Physical Rehabilitation Vienna, Austria; (2) Center for Medical Physics and Biomedical Engineering, Medical University of Vienna, Austria; (3) Dept. Physical Medicine and Rehabilitation, Wilhelminenspital Vienna, Austria

E-mail: stefan.loefler@wienkav.at

It was generally believed that no effective treatment was available for muscle that underwent severe atrophy due to chronic denervation. Under the Gutmann's ${ }^{1}$ view of the trophic influence of nerves on muscle, the effect of a mimicking approach, electrical stimulation, played an important role, but over the years the value of electrically stimulating the denervated muscle has been disputed because of the difficulties to obtain strong contraction by electrical stimulation and of its possible unfavorably effects on any remaining potential for reinnervation. In the last 15 years, we studied the possibility to effectively train permanently denervated human muscles by means of Functional Electrical Stimulation (FES). The results of the EU Project RISE ${ }^{2-4}$ show a new perspective in stimulating muscle fibers in the absence of nerves and after prolonged denervation, enabling: i) restoration of muscle fiber ultrastructure; ii) recovery of conduction velocity of the excitation-contraction apparatus up to a level that allows tetanic contractility; and thus iii) astonishingly recovery of fiber size, muscle mass and FESinduced force. Our training strategy is based on two combined stimulation programs. Within continuous clinical assessments, the stimulation parameters and training protocols should be progressively modified according to the patient's time span of denervation, the current condition of muscle and function. At the beginning of the treatment, biphasic stimulation impulses of very long-duration (120-150 $\mathrm{ms}, 60-75 \mathrm{~ms}$ per phase) at high intensity should be applied to improve membrane excitability and muscle structure. The next period of the routine daily training consists of combined stimulation patterns one eliciting single twitches (impulse duration of $120 \mathrm{~ms})$ and the other tetanic contractions $(2-3 \mathrm{~s}$ bursts with an impulse duration of $36-50 \mathrm{~ms}$ and impulse pause of $10 \mathrm{~ms}$ ). After tetanic contractility is achieved and the subject is able to provide full extension of the leg during stimulation of the quadriceps muscles, the ankle should be progressively loaded following the training theory for healthy people. Finally, few patients who have achieved a good muscle and functional condition can be able to stand and perform step-in-place and walking exercise with stimulation to train the cardiovascular system, upper body, sense of balance and thigh muscles.

1. Kern H, Hofer C, Mayr W, Carraro U. European Project RISE: Partners, protocols, demography. Basic Appl
Myol/European Journal of Translational Myology 2009;19:211-6.

2. Kern H, Carraro U, Adami N, et al. One year of homebased daily FES in complete lower motor neuron paraplegia: recovery of tetanic contractility drives the structural improvements of denervated muscle. Neurol Res 2010;32:5-12.

3. Kern $\mathrm{H}$, Carraro U, Adami $\mathrm{N}$, et al. Home-based functional electrical stimulation rescues permanently denervated muscles in paraplegic patients with complete lower motor neuron lesion. Neurorehabil Neural Repair 2010;24:709-21.

4. Kern H, Boncompagni S, Rossini K, et al. Long-term denervation in humans causes degeneration of both contractile and E-C coupling apparatus that can be reversed by functional electrical stimulation (FES). A role for myofiber regeneration? J Neuropath Exp Neurol 2004;63:919-31.

5. Boncompagni S, Kern H, Rossini K, et al. Structural differentiation of skeletal muscle fibers in the absence of innervation in humans. Proc Natl Acad Sci U S A 2007;104:19339-44.

$* * * * *$

P5 Rise4EU: Prevention of thromboembolism and fall in surgical units by volitional exercise, FES and FMS

Carraro U

IRCCS Fondazione Ospedale San Camillo, Venezia, Italy E-mail: ugo.carraro@unipd.it

Adult and aged population provide the vast majority of potential diseased people in Europe, the Americas and Japan. Their needs of mobility support and rehabilitation may be categorized as minimal (young light subjects during and after minor traumatic events), medium (old subjects with impairments in the normal life activity and sarcopenia), severe (advanced sarcopenia in oldest old subjects, oncology, neuromuscular and skeletal disorders, subjects weaning from long hospitalization for intensive care and/or heavy surgery) and extreme (severe neuromuscular disorders, permanent flaccid paraplegia due to lower motoneuron injury in the spine, cachexia due to severe nutritional, metabolic, oncologic and septic conditions). I will here briefly describe a comprehensive approach to help people with border line mobility and standing impairments, in particular those in surgical units that will need to rise soon after surgical interventions to decrease the risks of thrombo embolisms and falls. Surgical patients have a special need of voluntary mobility to minimize the need of personal to help them in every day toeletting and physiologic intestinal evacuation (catheterization solve the urology needs), but even more to stand-up as soon as possible after surgery. Indeed, dayhospital surgery although substantially increased is NOT possible for any surgical need. Any subject that is hospitalized arriving in a wheel-chair, is a potential subject that needs special care. The burden on the families and the services are increasing the number of aged people that use temporary or permanent wheel-chairs, more because it is easier and safer to be wheel-chaired to stand and walk. A program of rehabilitation in the days that precede 


\section{Eur J Transl Myol/Basic Appl Myol 2015; 25 (3): 145-182 \\ CIR-Myo News: Abstracts of the 2015 Spring Padua Muscle Days

\author{
Terme Euganee Padua (Italy), March 12 - 14, 2015
}

\section{Abstracts}

hospitalization or after hospitalization the surgery (patients spend some days for mandatory analyses to grant that the patients may stand the surgical act) may rescue borderline seniors from their "immobility" syndrome, and allow them to self-care with self-evident advantages for the individual and the Health System. The majority of these patients may reach independent (or supported, in particular if they are overweight and long-lasting disused) standing and walking by a program of volitional exercise to reactivate and strength enough their muscles to stand-up and walk. In particular cases, functional electrical stimulation (FES) and functional magnetic stimulation (FMS) may shorten the period of prestanding volitional activity.

U.C. thanks the Interdepartmental Research Center of Myology at the Department of Biomedical Sciences, University of Padova, Italy for collaboration and hospitality and the Ludwig Boltzmann Institute of Electrical Stimulation and Physical Rehabilitation of Vienna at the Department of Physical Medicine, Wilhelminenspital, Vienna, Austria for support and collaboration.

1. Battaglia G, Bellafiore M, Bianco A, et al. Effects of a dynamic balance training protocol on podalic support in older women. Pilot Study. Aging Clin Exp Res 2010;22:406-11.

2. Kern H, Carraro U, Adami N, et al. Home-based functional electrical stimulation rescues permanently denervated muscles in paraplegic patients with complete lower motor neuron lesion Neurorehabil Neural Repair. 2010; 24:709-721.

3. Gava P, Kern H, Carraro U. Age-related decline of muscle power in track and field master athletes indicates a lifespan of 110 years. Eur J Trans Myol/Basic Appl Myol 2013;23:45.

4. Gava P, Kern H, Carraro U Age-associated power decline from running, jumping \& throwing male master world-records. Exp Aging Res, 2015, In press.

$$
* * * * *
$$

P6. Assessment of muscles, connective and fat tissue, using $\mu \mathrm{CT}$ data: feasibility study for meat quality control

$$
\text { Gargiulo P (1,2), Örlygsson G (3), Rizzi C (4,5), Carraro U }
$$

(1) Dept. of Science, Landspitali University Hospital, Reykjavik; (2) Dept. of Biomedical Engineering, University of Reykjavik, (3) Dept. of Materials, Biotechnology and Energy, Innovation Center Iceland, Reykjavik, Iceland; (4) Dept. of Biotechnology, University of Verona, Italy; (5) Istituto Italiano Assaggiatori Carne, Torri del Benaco, Verona, Italy; (6) IRCCS Fondazione Ospedale San Camillo, Venezia, Italy.

Email: paologar@landspitali.is

Meat eating traits, such as tenderness and juiciness, are known to be linked to total fat levels, and to the associations between intra-muscular connective and fat distribution. The visual appearance of the fat could additionally affect the consumers overall acceptability of the product and therefore the choice and the selection of meat products before buying .,2 Bioimaging, image processing and 3D modeling has showed a fundamental role in assessing denervated muscles during electrical stimulation. ${ }^{3,4}$ Similar techniques have been recently employed to monitor Extracellular Matrix Mineralization in biological scaffolds using X-ray $\mu \mathrm{CT}$ technology.5 A combination of these two approaches was used to analyse meat samples with the General Electric nanotom x-ray $\mu \mathrm{CT}$ system, this system has $200 \mathrm{~nm}$ detail detectability. The aim is to study the feasibility of developing an alternative methodology for meat quality assessment based on image processing that could be in the future correlated with specific sensory analysis. A salted-smokedfermented meat sample (Tiroler-speck, a typical product of the north part of Italy) of $10 \times 10 \times 1 \mathrm{~mm}$ was scanned with step of $3,5 \mu \mathrm{m}$ using $\mu \mathrm{ct}$ technology. The $\mu$ ct data scan data are imported into a special image processing and editing computer program called MIMICS. ${ }^{6}$ In this software environment we isolate: muscles, intra muscular connective tissue and fat tissues. the discrimination between tissues is possible because of the different linear attenuation coefficient and consequent gray value intensity. False colors are assigned to the muscles, connective and fat tissues within the sample and the amount of each tissue have been quantified. ${ }^{7}$ Moreover, it was also possible to isolate few single muscle fibers of 40-60 $\mu \mathrm{mm}$ diameters. Correlation of the 3D color analysis of each sample product with expectations of buyers may open large application to the imaging approach.

U.C. thanks the Interdepartmental Research Center of Myology at the Department of Biomedical Sciences, University of Padova, Italy for collaboration and hospitality and the Ludwig Boltzmann Institute of Electrical Stimulation and Physical Rehabilitation of Vienna at the Department of Physical Medicine, Wilhelminenspital, Vienna, Austria for support and collaboration.

1. Frisullo P, Marino R, Laverse J, et al. Assessment of intramuscular fat level and distribution in beef muscles using X-ray microcomputed tomography. Meat Science 2010;85:250-5.

2. Lambe NR, Navajas EA, Fisher AV, et al. Prediction of lamb meat eating quality in two divergent breeds using various live animal and carcass measurements Meat Science 2009;83:366-75.

3. Gargiulo P, Helgason T, Ingvarsson $P$, et al. Medical Image Analysis and 3-D Modeling to Quantify Changes and Functional Restoration in Denervated Muscle Undergoing Electrical Stimulation Treatment. Humancentric Computing and Information Sciences 2012;2:10.

4. Gargiulo P, Kern H, Carraro U, et al. Quantitative color three-dimensional computer tomography imaging of human long-term denervated muscle. Neurol Res 2010;32:13-9.

5. Czenek A, Conte G. Lieder R, et al. Monitoring Extracellular Matrix Mineralization Processes in Calcium Phosphate Scaffolds using X-ray $\mu \mathrm{CT}$ Technology and 3D Modeling Methods. 25th European Conference on Biomaterials, ESB 2013.

6. Materialise. Product: MIMICS. Available at: http://www.materialise.com. Accessed in October 2013.

7. Gargiulo P, Reynisson PJ, Helgason B, Kern H, Mayr W, Ingvarsson P, Helgason T, Carraro U. Muscle, tendons, and bone: structural changes during denervation and FES treatment. Neurol Res 2011;33:750-8. 
Abstracts

Index

\begin{tabular}{|c|c|}
\hline Adamo S & 175 \\
\hline Albiero M & 162 \\
\hline Angelini A & 169 \\
\hline Annibali D & 159 \\
\hline Argenton F & 155 \\
\hline Armani A & 160 \\
\hline Atherton PJ & 146 \\
\hline Baba A & 156,175 \\
\hline Bachna-Rotter S & 164,165 \\
\hline Baumbusch K & 165 \\
\hline Beltrame V & 147 \\
\hline Berardi E & 159 \\
\hline Bernardi P & 155 \\
\hline Bertaggia E & 160 \\
\hline Bertin E & 151 \\
\hline Bertolucci F & 156,161 \\
\hline Betto R & 154 \\
\hline Bianchini E & 154 \\
\hline Bijak M & $166,167,167$ \\
\hline Blaauw B & 155,162 \\
\hline Böhmerová L & 161 \\
\hline Bonaldo P & 155 \\
\hline Boncompagni S & $152,171,176$ \\
\hline Borschel G & 167 \\
\hline Bradley K & 157 \\
\hline Braghetta P & 155 \\
\hline Burggraf $\mathrm{S}$ & 159,179 \\
\hline Cancellara P & 148 \\
\hline \multicolumn{2}{|l|}{ Carraro U 14} \\
\hline Casarin A & 162 \\
\hline Cassano M & 159 \\
\hline Castellani C & 169 \\
\hline Cavallari P & $166,166,166$ \\
\hline Chiang C & 167 \\
\hline Chisari C & 156,161 \\
\hline Coletti D & 175 \\
\hline Conte M & 160 \\
\hline Coran A & 147 \\
\hline Costamagna D & 159 \\
\hline Costelli P & 159 \\
\hline Cudia P & 156,175 \\
\hline Cvečka J & 159,161 \\
\hline \multicolumn{2}{|c|}{ D'Errico-Grigioni A 160} \\
\hline D’Onofrio L & 171 \\
\hline Dalise $S$ & 156,161 \\
\hline Danieli-Betto D & 151 \\
\hline De Conti C & 155 \\
\hline De Coppi P & 151 \\
\hline Degiovanni A & 160 \\
\hline Desbats M & 162 \\
\hline Deshmukh A & 148 \\
\hline Di Fonso A & 171 \\
\hline
\end{tabular}

\begin{tabular}{|c|c|}
\hline Di Grazia A & 159 \\
\hline Di Muzio A & 176 \\
\hline Edmunds K & 145 \\
\hline Fahnestock M & 167 \\
\hline Foerster G & 165 \\
\hline Forcina L & 158 \\
\hline Forte $\mathrm{M}$ & 155 \\
\hline Fracasso F & 178 \\
\hline Franceschi C & 160 \\
\hline Franchi MV & 146 \\
\hline Frangež R & 168 \\
\hline Franzin C & 151,169 \\
\hline Friedrich G & 164,165 \\
\hline Fröhlich-Sorger E & 165 \\
\hline Fruhmann $\mathrm{H}$ & 159 \\
\hline Galli L & 176 \\
\hline Gargiulo P & $145,152,180$ \\
\hline Gava P & 157 \\
\hline Gelbmann L & 149 \\
\hline Germinario E & 151 \\
\hline Gerstenberger C & 164,165 \\
\hline Gobbo V & $149,151,152$ \\
\hline Gordon $\mathrm{T}$ & 167 \\
\hline Gorza L & 169 \\
\hline Graupe D & 174 \\
\hline Graupp M & 165 \\
\hline Grosemans H & 159 \\
\hline Gudnason V & 145 \\
\hline Gugatschka M & 164,165 \\
\hline Hagen R & 165 \\
\hline Halldorsdottir G & 173 \\
\hline Haller M & 167 \\
\hline Hamar D & 159,161 \\
\hline Harnisch W & 165 \\
\hline Helgason $\mathrm{T}$ & 173 \\
\hline Hockerman G & 157 \\
\hline Hofer Ch & 176 \\
\hline Iodice P & 176 \\
\hline Jarvis JC 147 , & $147,164,167,167$ \\
\hline Joseph A-M & 178 \\
\hline Kemp SWP & 167 \\
\hline Kern H & $152,157,159,161,162$ \\
\hline Khobragade N & 174 \\
\hline Kiesler K, & 165 \\
\hline Kiper P & 175 \\
\hline Kovárová J & 161 \\
\hline Krenn M & 150,172 \\
\hline Kristinsson $\mathrm{K}$ & 173 \\
\hline Lacatena A & 175 \\
\hline Lanmüller H & $166,167,167$ \\
\hline Leeuwenburgh C & 162,178 \\
\hline Lezza AMS & 170,178 \\
\hline
\end{tabular}

\begin{tabular}{|c|c|}
\hline Lindenthaler $\mathrm{W}$ & 165 \\
\hline Löfler S & $159,162,176179$ \\
\hline Maganaris C & 146 \\
\hline Mammucari C & 159,163 \\
\hline Mancinelli R & 175 \\
\hline Mann M & 148 \\
\hline Maraldi NM & 155 \\
\hline Marcante A & $152,156,175,177$ \\
\hline Martinuzzi A & 155 \\
\hline Marzetti E & 169 \\
\hline Masiero S & $152,156,175,177$ \\
\hline Matthews M & 154 \\
\hline Mayr W & $150,172,177$ \\
\hline Mayr W & 179 \\
\hline Merico A & 156,175 \\
\hline Merlini L & 155 \\
\hline Moedlin M & 179 \\
\hline Moresi V & 175 \\
\hline Mosole S & $151,152,159,179$ \\
\hline Mueller AH & 165 \\
\hline Murgia M & 148 \\
\hline Musarò A & 158,159 \\
\hline Nagaraj N & 148 \\
\hline Narici MV & 146,160 \\
\hline Örlygsson G & 180 \\
\hline Ortiz C & 159 \\
\hline Ortolan P & 147 \\
\hline Palma E & 155 \\
\hline Panjan A & 173 \\
\hline Paoli A & 169 \\
\hline Papini E & 169 \\
\hline Patruno M & 177 \\
\hline Pečlin P & 168 \\
\hline Pelosi L & 158 \\
\hline Perini I & 159 \\
\hline Perosa M & 161 \\
\hline Pesce V & 178 \\
\hline Petersen H & 171,172 \\
\hline Picca A & 178 \\
\hline Piccione F & $152,156,175,177$ \\
\hline Piccoli M & 151 \\
\hline Pigna E & 175 \\
\hline Pond A & $152,157,177$ \\
\hline Pototschnig C & 165 \\
\hline Pozzobon M & $151,169,177$ \\
\hline Pratt E & 157 \\
\hline Praznikar J & 161 \\
\hline Pribyl J & 149 \\
\hline Protasi F & $152,164,171,176$ \\
\hline Quinlivan R & 153 \\
\hline Rabie A & 174 \\
\hline Rafolt D & 172 \\
\hline Ravara B & $149,151,169$ \\
\hline
\end{tabular}


European Journal of Translational Myology/Basic Applied Myology 2015; 25: 145-182

Proceedings of the 2013 Spring Padua Muscle Days

Terme Euganee, Padova (Italy), March 15 - 17, 2013

$\begin{array}{ll}\text { Reeves ND } & 146 \\ \text { Reggiani C } & 148 \\ \text { Rizzi C } & 180 \\ \text { Rizzo E } & 155 \\ \text { Romanello V } & 162 \\ \text { Rosa E } & 167 \\ \text { Rossi B } & 156,161 \\ \text { Rossi S } & 175 \\ \text { Roy S } & 155 \\ \text { Rozman J } & 168,177 \\ \text { Sabatelli P } & 155 \\ \text { Sacchetto R } & 154 \\ \text { Salmons S } & 145 \\ \text { Salviati L } & 162 \\ \text { Salvioli S } & 160 \\ \text { Sampaolesi M } & 159 \\ \text { Sandonà D } & 154 \\ \text { Sandri M } & 159,160,162 \\ \text { Šarabon N } & 159,161,173 \\ \text { Schiaffino S } & 148 \\ \text { Schiavone M } & 155 \\ \text { Schickhofer P } & 161 \\ \text { Schils SJ } & 148,149 \\ \text { Schlager HG } & 164,165 \\ \text { Schmoll M } & 166,167,167\end{array}$

$\begin{array}{ll}\text { Schoenen F } & 155 \\ \text { Schöffski P } & 159 \\ \text { Scorrano L } & 162,164 \\ \text { Sedliak M } & 159,161 \\ \text { Sigurdardottir JS } & 173 \\ \text { Sigurdsson S } & 145 \\ \text { Sigurthorsson SP } & 173 \\ \text { Šileikyte J } & 155 \\ \text { Slavin KV } & 174 \\ \text { Smith K } & 146 \\ \text { Soderling I } & 157 \\ \text { Soriano ME } & 162 \\ \text { Sorrentino V } & 176 \\ \text { Stephenson RS } & 147 \\ \text { Stramare R } & 147 \\ \text { Tagliavini F } & 155 \\ \text { Tasca E } & 175 \\ \text { Tavano R } & 169 \\ \text { Tezze C } & 162 \\ \text { Thiene G } & 169 \\ \text { Tirpáková V } & 159,161 \\ \text { Tramonti C } & 156,161 \\ \text { Trevisi E } & 155 \\ \text { Tuninetti D } & 174 \\ \text { Ultimo S } & 159\end{array}$

$\begin{array}{lc}\text { Unger E } & 166,167,167 \\ \text { Vajda M } & 161 \\ \text { Varanita T } & 162 \\ \text { Vasuri F } & 160 \\ \text { Vavla M } & 155 \\ \text { Verhagen Metman L } 174 \\ \text { Vescovo G } & 169 \\ \text { Vettor R } & 169 \\ \text { Vindigni V } & 152,177 \\ \text { Vizzaccaro E } & 158 \\ \text { Vrecl M } & 168 \\ \text { Wang W-H } & 157 \\ \text { Willand MP } & 167 \\ \text { Wozniak A } & 159 \\ \text { Zampieri S } & 151,152,159, \\ & 162,177,178 \\ \text { Zanato R } & 147 \\ \text { Zeiler M } & 148 \\ \text { Zhang JZ } & 167 \\ \text { Zidar J } & 150,177 \\ \text { Zulian S } & 155 \\ \text { Žužek MC } & 168\end{array}$

Schmoll M $\quad 166,167,167$

Abstracts 